\title{
Phytosociological analysis of basophilic Scots pine forests in the Southeastern Alps
}

\author{
Andrej Rozman ${ }^{1}$ (i) , Igor Dakskobler² \& Urban Šilc ${ }^{3}$ (i)
}

Key words: phytosociology, synsystematics, Scots pine, Black pine, forest, Slovenian Alps, Natura 2000, habitat type.

Ključne besede: fitocenologija, sinsistematika, rdeči bor, črni bor, gozd, slovenske Alpe, Natura 2000, habitatni tip.
Received: 4. 18. 2019

Revision received: 25.10 .2019

Accepted: 30. 10. 2019

\begin{abstract}
Based on hierarchical classification of more than 300 phytosociological relevés of basophilic black and (or) Scots pine communities in the Southern, Eastern and Southeastern Alps we described a new association Rhodothamno chamaecistiPinetum sylvestris, into which we classify stands that have until now been discussed in the framework of subassociations Fraxino orni-Pinetum nigrae pinetosum sylvestris, laricetosum deciduae and (partly) caricetosum humilis, and are floristically slightly similar also to certain forms of the association Erico-Pinetum sylvestris. The stands of the new association are for now classified into Natura 2000 habitat type Southeastern-European Pinus sylvestris forests (91R0), within it we propose a special habitat subtype Southeastern-Alpine Scots pine forests, and into a new forest site type Southeastern-Alpine Scots pine forest. At the contact of the Julian and Dinaric Alps we described a new subassociation Genisto januensis-Pinetum sylvestris campanuletosum cespitosae, which comprises also a Natura 2000 species Primula carniolica.
\end{abstract}

Izvleček

Na podlagi hierarhične klasifikacije več kot 300 fitocenoloških popisov bazoljubnih združb črnega in (ali) rdečega bora v Južnih, Vzhodnih in Jugovzhodnih Alpah smo opisali novo asociacijo Rhodothamno chamaecistiPinetum sylvestris, v katero uvrščamo sestoje, ki smo jih do zdaj obravnavali v okviru subasociacij Fraxino orni-Pinetum nigrae pinetosum sylvestris, laricetosum deciduae in (deloma) caricetosum humilis, floristično pa so nekoliko podobni tudi nekaterim oblikam asociacije Erico-Pinetum sylvestris. Sestoje nove asociacije za zdaj uvrščamo v Natura 2000 habitatni tip Jugovzhodnoevropski gozdovi rdečega bora (91R0), kot poseben podtip Jugovzhodnoalpski gozdovi rdečega bora in $\mathrm{v}$ nov gozdni rastiščni tip Jugovzhodnoalpsko rdečeborovje. Na stiku Julijskih Alp in Dinarskega gorstva smo opisali novo subasociacijo Genisto januensis-Pinetum sylvestris campanuletosum cespitosae, v kateri uspeva tudi Natura 2000 vrsta Primula carniolica.

\footnotetext{
1 Biotechnical Faculty of the University in Ljubljana, Department of Forestry and Renewable Forest Resources, Večna pot 83, 1000 Ljubljana, Slovenia. E-mail: andrej.rozman@bf.uni-lj.si

2 Scientific Research Centre of the Slovenian Academy of Sciences and Arts, Institute of Biology, Regional Unit Tolmin, Brunov drevored 13, 5220 Tolmin, Slovenia E-mail: Igor.Dakskobler@zrc-sazu.si

3 Scientific Research Centre of the Slovenian Academy of Sciences and Arts, Institute of Biology, Novi trg 2, 1000 Ljubljana, Slovenia. E-mail: Urban@zrc-sazu.si
} 


\section{Introduction}

Scots pine (Pinus sylvestris) and black pine (Pinus nigra) are very different in terms of their distribution area and ecological characteristics. Both are pioneers and occur as such in forests in most of Slovenia, sometimes they are even planted. Their natural sites are frequently (Scots pine) or almost exclusively (black pine) on very steep dolomite slopes where other tree species cannot compete. Such natural forest stands have long been the subject of phytosociological research: Aichinger (1933), Schmid (1936), Tomažič (1940), M. Wraber (1960), T. Wraber (1979), Piskernik (1979), Dakskobler (1998a, b, 1999, 2006), Accetto (1999, 2001, 2008, 2010, 2013, 2015), Cimperšek (2005), Zupančič \& Žagar (2010), Dakskobler et al. (2015). According to the findings from neighbouring Alpine regions of Italy and Austria (Poldini 1969, 1982, Poldini \& Vidali 1999, Martin-Bosse 1967, Franz 2002, Eichberger et al. 2004, 2007a,b, E. \& S. Pignatti 2014, 2016), black and (or) Scots pine stands on very steep, rocky dolomite sites and in erosion areas in the Alpine part of Slovenia have so far been classified into the association Fraxino orni-Pinetum nigrae, including the more or less pure Scots pine stands that are treated at the rank of subassociations pinetosum sylvestris, laricetosum deciduae and caricetosum humilis. Ecologically similar Scots (and black) pine stands outside the Alps, in the northern part of the Dinaric Alps, are classified into the association Genisto januensis-Pinetum sylvestris (including subassociation pinetosum nigrae), whereas black pine stands are classified into several associations (Carici sempervirentis-Pinetum nigrae, Daphno alpinae-Pinetum nigrae, Primulo carniolicae-Pinetum nigrae, Thymo praecocis-Pinetum nigrae).

With a phytosociological analysis of extensive relevé material on basophilic Scots and black pine communities in the Southern, Southeastern and Eastern Alps (triggered by the applied research project by Šilc et al. 2017) we are looking into whether it makes sense to differentiate predominantly pure Scots pine stands that sporadically occur in the Julian Alps, the Kamnik-Savinja Alps and in the Karavanke Mts. from the predominantly pure black pine stands on similar sites in the Julian Alps and western Karavanke Mts., rarely also in the Kamnik-Savinja Alps (the Kokra Valley). Given that they occur on similar sites, can they be treated within the habitat type (Sub)Mediterranean pine forests with endemic black pines $\left(9530^{*}\right)$, even though they are actually Scots pine stands in the Southern-Alpine region? Is it correct for pure Scots pine stands where black pine does not occur at all to be classified into the association named explicitly after black pine - Fraxino orni-Pinetum nigrae? Is the basophilic Scots pine community in the Southeastern Alps ecologically and floristically different from basophilic Scots pine communities elsewhere in the Alps that are classified into the association Erico-Pinetum sylvestris?

\section{Methods}

A total of 389 relevés of Pinus sylvestris and Pinus nigra stands are stored in the FloVegSi database (Seliškar et al. 2003) and Vegetation database of Slovenia (Šilc 2012). Only 308 of these relevés were used in our analysis, because others were too different in ecological terms. The stand layers recorded on sites were merged into four main layers: the tree layer (E3), the shrub layer (E2), the herb layer (E1) and the moss layer (E0), where two shrub and two tree sublayers were merged in one shrub and one tree layer using Jennings et. al. (2009) equation:

$C_{i}=\left[1-\prod_{j=1}^{n}\left(1-\frac{\% \operatorname{cov} j}{100}\right)\right] \times 100$

where $\operatorname{cov} j$ is species cover in sublayer $j$.

The relevés were compared by means of hierarchical classification using the Unweighted average linkage clustering method (UPGMA) and Wishart's similarity ratio. For this purpose, the original Braun-Blanquet cover values were converted into percentages and transformed by square root. In addition we analysed the Wishart's dissimilarity matrix using the principal coordinate analysis (PCoA) method. The ecological variables obtained from the relevés and weighted mean Pignatti ecological indicator values as estimates of ecological variables (Pignatti 2005) were added to the PCoA plot by regression as passive variables. Species accumulation curves (SAC) were used to compare diversity in different syntaxa. In identifying the indicator species of the syntaxa we used the Indicator Value Index (Dufrene \& Legendre 1997). Numerical analyses were made with the software package SYN-TAX (Podani 2001) and R (R Core Team 2018), using the package "vegan" (Oksanen et al. 2018).

The variable northness, calculated as $(\cos ($ azimuth $)+1) / 2$, reflects the heat received by the site and moves in the range from 0 (south exposition) to 1 (north exposition), symmetrically over west and east slopes.

Climate data (precipitation volume and mean temperature) were obtained from high resolution raster maps provided by the Environmental Agency of the Republic of Slovenia, Ministry of the Environment and Spatial Planning (http://www.arso.gov.si/).

The nomenclatural source for the names of vascular plants are the Mala flora Slovenije (Martinčič et al. 2007) and Flora alpina (Aeschimann 2004a,b). Martinčič 
$(2003,2011)$ is the nomenclatural source for the names of mosses and Suppan et al. (2000) is the nomenclatural source for the names of lichenized fungi. The determination of mosses and lichenized fungi is not always reliable. The nomenclatural sources for the names of syntaxa are Theurillat (2004), Šilc \& Čarni (2012) and Mucina et al. (2016). Buser (2009) is the source of data on the geological bedrock and the source for the nomenclature of soil types is Urbančič et al. (2005).

\section{Results and Discussion}

\section{Relations of Pinus sylvestris forests in the SE Alps to similar Pinus communities in the SE Alps and the North Dinaric Alps}

In the first step we compared, based on an extensive database (21 tables, 308 relevés) that comprised already published phytosociological relevés of basophilic pine forests of the Sothern and Southeastern Alps (Martin-Bosse 1967, T. Wraber 1979, Poldini \& Vidali 1999, Dakskobler 2006, Zupančič \& Žagar 2010, E. \& S. Pignatti 2014, 2016) and our unpublished relevés (Dakskobler, mscr., Rozman, mscr.), the floristic composition of basophilic communities with dominant black and (or) Scots pine (Appendix 1). Scots pine relevés from the Southeastern Alps grouped separately from the southeastern-Alpine black pine community (Fraxino orni-Pinetum nigrae) and the Alpine Scots pine community (Erico-Pinetum sylvestris) (Figure 1).

Based on these comparisons we were able to isolate a group of 52 relevés with dominant Scots pine (Table 1). These included several already published relevés from the Julian Alps, the Karavanke Mts. and the Kamnik-Savinja Alps (T. Wraber 1979: Pinetum austroalpinum pinetosum sylvestris; Dakskobler 2006: Fraxino orni-Pinetum nigrae pinetosum sylvestris var. Larix decidua; Zupančič \& Žagar 2010: Fraxino orni-Pinetum nigrae laricetosum et caricetosum humilis) as well as new relevés (Rozman, Dakskobler), mainly from the Karavanke Mts. To ensure an adequate syntaxonomic designation we conducted an-
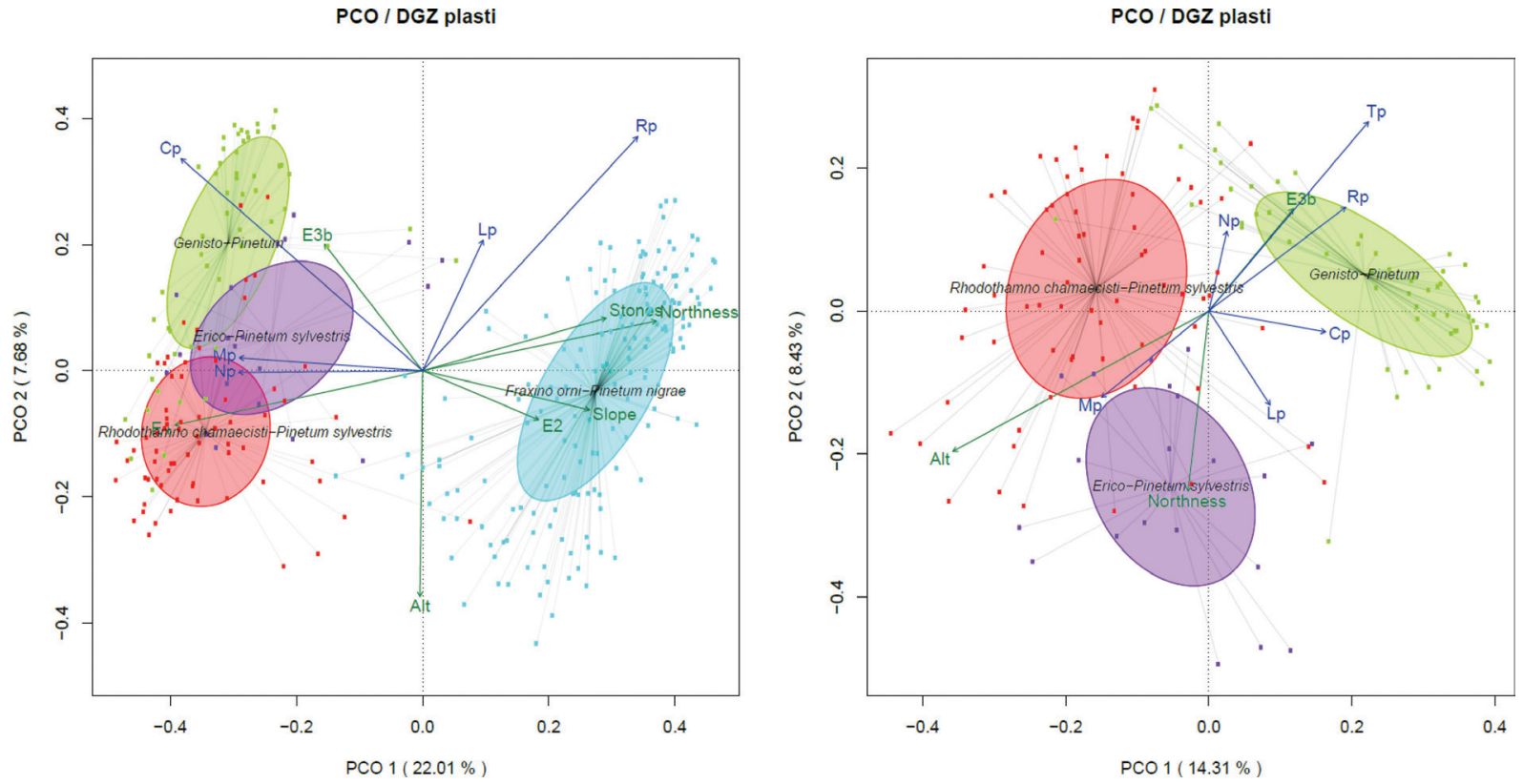

Figure 1: Two principal coordinate analysis (PCoA) ordination plots. The left ordination diagram comprises all Scots and black pine relevés, whereas the right only features the relevés with Pinus sylvestris as the dominant species. The ellipses represent the standard deviation of the relevés belonging to a particular pine syntaxon. The first two axes explain $22.01 \%$ and $7.68 \%$, respectively, of the variability in data. Arrows represent ecological variables (E3b-upper tree layer coverage, E2-shrub layer coverage E1-herb layer coverage, Alt-altitude, Slope, Stoniness, Northness) or their estimates (weighted mean Pignatti ecological indicator values: Lp-light, Cp-continentality, Rp-soil reaction, Mp-moisture, Np-nitrogen, Tp-temperature). Slika 1: Dve sliki ordinacije PCoA. Leva slika predstavlja ordinacijo vseh obravnavanih popisov rdečega in črnega bora, desna slika je ordinacija samo združb rdečega bora. Elipse predstavljajo standardni odklon popisov posameznih tabel borovih združb. Prvi dve osi ordinacije pojasnita $22.01 \%$ in $7.68 \%$ variabilnosti. Puščice predstavljajo ekološke spremenljivke (E3b-zastrtost zgornje drevesne plasti, E2-zastrtost grmovne plasti, E1- zastrtost zeliščne plasti, Alt-nadmorska višina, Slope-nagib, Stoniness-kamnitost, Northness-severnost) or their estimates (tehtana povprečja Pignattijevih ocen ekoloških dejavnikov: Lp-svetloba, Cp-kontinentalnost, Rp-reakcija tal, Mp-vlažnost, Np-dušik, Tp-temperatura). 
other comparison with a synoptic table featuring eight syntaxa with dominant Pinus sylvestris from the wider area (Table 3, Figure 2).

Comparison shows that the studied stands are the most similar to the stands of the subassociation Fraxino orniPinetum nigrae pinetosum sylvestris from NE Italy and stands of the association Erico-Pinetum sylvestris from the Dolomites in northern Italy. Similarity with stands of the association Genisto januensis-Pinetum sylvestris from western Slovenia is less pronounced, and even less similar are different forms of the association Erico-Pinetum sylvestris from Austria, also to the stands of the subassociation ostryetosum carpinifoliae Franz 2002. Differences between the compared syntaxa are demonstrated also by the analysis of diagnostic species of individual phytosociological groups (Table 4). Differences are evident especially in the relative proportions of diagnostic species of classes Erico-Pinetea, Vaccinio-Piceetea, Festuco-Brometea in Elyno-Seslerietea, partly also in relative proportions of diagnostic species of orders Fagetalia sylvaticae and Quercetalia pubescenti-petraeae and classes Rhamno-Prunetea, Trifolio-Geranietea and Thlaspietea rotundifolii. Sørensen's similarity index (1948) showed that floristic similarity between the three syntaxa (RcPs-Si, FPnps and EPs-Do) is $55 \%$, whereas Jaccard's index was $40 \%$, which allows us to classify their stands into the same association (either Erico-Pinetum sylvestris or Fraxino orni-Pinetum nigrae). Classification into the association Fraxino orni-Pinetum nigrae (subassociations pinetosum sylvestris) is problematic because the studied stands do not comprise black pine. Its classification into a habitat type would mean that Scots pine stands are classified into a black pine habitat type. For other related tree species such as oak, we normally distinguish sessile oak communities from pubescent oak or Turkey oak communities - both at the level of association and habitat types (Šilc \& Čarni 2012, Kutnar et al. 2012). Contrary to previous studies in the past (T. Wraber, L. Poldini, M. Zupančič) we believe that a Scots pine community should be named after this species and not after black pine. Another, more appropriate option would be to treat these stands as a special southeasternAlpine geographical variant of the association Erico-Pinetum sylvestris, but with high number of good differential species. However, as this association evidently comprises very diverse stands (as evident in the synoptic table of this association for the territory of Austria and surroundings, Eichberger et al. 2007b) that all comprise Scots pine and winter heath (Erica carnea), and since geographical variant is not a rank regulated by the Code of Phytosociological Nomenclature (Weber et al. 2000), the performed comparisons allow for the third option - description of the new association Rhodothamno-Pinetum sylvestris. Its

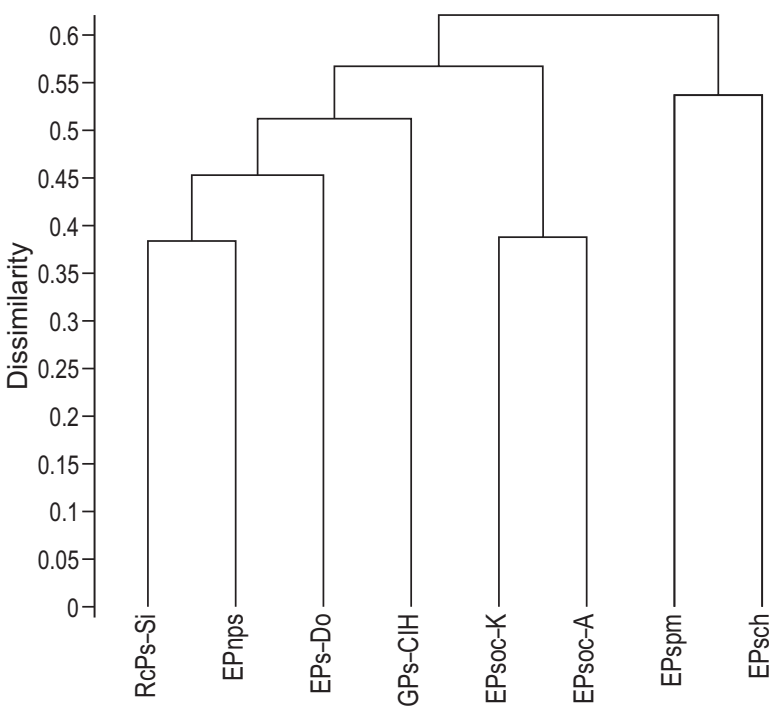

Figure 2: Dendrogram of eight communities with dominant Pinus sylvestris in the Southern, Eastern and Southeastern Alps, UPGMA, 1-similarity ratio (See legend below).

Slika 2: Dendrogram osmih združb s prevladujočim rdečim borom v Južnih, Vzhodnih in Jugovzhodnih Alpah, UPGMA, komplement Wishartovega koeficienta podobnosti (glej legendo spodaj).

RcPs-Si Rhodothamno-Pinetum sylvestris, this article, Table 1 (52 relevés)

FPnps Fraxino orni-Pinetum nigrae pinetosum sylvestris, NW Italy, (Poldini \& Vidali 1999, Table 2, relevés 18-28 (11 relevés);

EPs-Do Erico-Pinetum sylvestris, N Italy, Dolomites, E. \& S. Pignatti 2016, Association Table 5.2 (20 relevés);

GPs-CIH Genisto-Pinetum sylvestris, W Slovenia, Cerkno and Idrija Hills, this article, Table 2 ( 55 relevés);

EPsoc-K Erico-Pinetum sylvestris ostryetosum carpinifoliae, Carinthia, Austria, Franz 2002, Table 34 (19 relevés);

EPspm Erico-Pinetum sylvestris pinetosum mugo, Austria (Eichberger et al. 2007 b, Table 29, Column 2) (18 relevés);

EPsch Erico-Pinetum sylvestris caricetosum humilis, Austria and partly neighbouring countries (Eichberger et al. 2007 b, Table 29, Column 4) (36 relevés);

EPsoc-A Erico-Pinetum sylvestris ostryetosum carpinifoliae, Austria (Eichberger et al. 2007 b, Table 29, Column 7) (27 relevés).

diagnostic species are Scots pine (Pinus sylvestris), which is the edifier of the community, as well as Rhodothamnus chamaecistus, Laserpitium peucedanoides, Betonica alopecuros, Campanula cespitosa, Valeriana saxatilis, Hieracium porrifolium, Cyclamen purpurascens, Anemone trifolia, Helleborus niger, Salix glabra, Chamaecytisus purpureus and Fraxinus ornus. The listed species characterise intrazonal southeastern-Alpine Scots pine community on extreme rocky sites in the belt of Illyrian beech forests from the alliance Aremonio-Fagion on very steep, sunny and shady dolomite slopes and jags on shallow initial soil in the montane and altimontane belt (from 530 to $1350 \mathrm{~m}$ a.s.1.). The eastern-Alpine species Rhodothamnus chamaecistus is very rare in the relevés of a similar Scots pine 
community from northern and northeast Italy, but these relevés are within its distribution area. Other diagnostic species of the association Rhodothamno-Pinetum sylvestris occur in the Scots pine community from northern and northeast Italy, e.g. Campanula cespitosa, Betonica alopecuros, Fraxinus ornus, Chamaecytisus purpureus, Valeriana saxatilis. Some of them occur also in the stands of the Illyrian (pre-Alpine-Dinaric) association Genisto januensisPinetum sylvestris, especially on the northwestern border of its distribution area, in the foothills of the Southern Julian Alps (Table 2). This peripheral form is characterised by several (southeastern-Alpine)-Illyrian species (Genista januensis, Scabiosa hladnikiana, Salvia pratensis subsp. saccardiana, Phyteuma scheuchzeri subsp. columnae, Primula carniolica) as well as Campanula cespitosa, Allium ericetorum, Hieracium porrifolium and Chamaecytisus purpureus, which are frequent also in the southeastern-Alpine Scots pine community. The entire floristic diversity is nevertheless evident, with the proportion of diagnostic species of the class Vaccinio-Piceetea in the Alpine Scots pine community totalling $11.7 \%$ and only $3.6 \%$ in the northernDinaric-pre-Alpine community, whereas the latter comprises a substantially higher proportion of diagnostic species of the class Festuco-Brometea and order Quercetalia pubescenti-petraeae. Larix decidua is a good differential species of the southeastern-Alpine Scots pine community against the pre-Alpine-Dinaric community.

The new association is divided into two subassociations. The stands of the slightly more thermophilous subassociation euphorbietosum amygdaloidis occur at on average lower elevations and slightly warmer sites. The differential species of the subassociation are Euphorbia amygdaloides, Euphorbia cyparissias and Teucrium chamaedrys. We distinguish between two variants. The variant with Gentiana asclepiadea characterises stands that are very atypical for the studied community - their tree layer is dominated by larch and spruce rather than Scots pine (one of the relative differential species is also Valeriana tripteris). These stands indicate a certain similarity with stands of associations Rhodothamno-Laricetum and Aposerido-Piceetum, which indicates that they are unlikely to be primary Scots pine stands. The floristic composition of the variant with Viola hirta (its differential species are also Vincetoxicum hirundinaria and Brachypodium rupestre) possibly indicates the effects of past grazing of small ruminants. The localities of the relevés are in the western Karavanke Mts. and in the Savinja Alps, two relevés are also from the Julian Alps (Mala Pišnica). Differential species of the subassociation sorbetosum aucupariae are Rhododendron hirsutum, Sorbus aucuparia, Vaccinium myrtillus and Juniperus communis. Sesleria caerulea and species of the class Elyno-Seslerietea in particular also have a certain differential value. This is evident in the indicator species analysis (Table 5). The species we identified as indicator species were those whose IndVal value was characteristic $(\mathrm{P}<0.05)$ for a particular subassociation. Table 5 shows number of indicator species of particular phytosociological groups in each subassociation. Indicator species in the first subassociation do not comprise species of the class Elyno-Seslerietea, but feature more species of the class Festuco-Brometea, whereas the situation is reversed in the second subassociation.

Relevés of the subassociation sorbetosum aucupariae characterise comparatively colder sites with mor rendzina at slightly higher elevations. The localities of the relevés are in the eastern Karavanke Mts. and in the Julian Alps, only one relevé is from the Savinja Alps.

Scots pine stands in Table 2 are classified into the association Genisto januensis-Pinetum sylvestris and the new subassociation campanuletosum cespitosae. The differential species of the subassociation are the eastern-Alpine species Campanula cespitosa and the south-European montane species Allium ericetosum, which indicate a certain similarity with the Alpine Scots pine community and the vicinity of the Julian Alps. These two species are not mentioned in the phytosociological table of the original description of this association (Tomažič 1940). Currently known distribution of the stands of the new subassociation in Slovenia is shown in Appendix 2. The stands were inventoried in the Cerkno and the Idrija Hills, which partly belong to the foothills of the Julian Alps (preAlpine phytogeographical region) and partly to the Dinaric Alps (Dinaric phytogeographical region), and in the Trebuša Valley (Dinaric Alps) at elevations spanning 300 to $920 \mathrm{~m}$, on steep to very steep sunny and shady dolomite slopes.

\section{Synsystematic classification of newly described communities}

According to the syntaxonomic system of higher units (Šilc \& Čarni 2012, Mucina et al. 2016), studied Scots pine forests can be classified as follows:

\section{Erico-Pinetea Horvat 1959}

Erico-Pinetalia Horvat 1959 nom. conserv. propos.

Erico carneae-Pinion Br.-Bl. in Br.-Bl. et al. 1939 nom. invers. propos.

(Fraxino orni-Pinion nigrae-sylvestris Zupančič 2007, group of basophilic Pinus nigra and (or) Pinus sylvestris communities in the Southeastern Alps and northern Dinaric Alps)

Rhodothamno chamaecisti-Pinetum sylvestris ass. nov. hoc loco, nomenclatural type, holotypus, is relevé 17 in Table 1 . 
Syn: Pinetum austroalpinum (Aich. 1933) Br.Bl. et Siss 1939 pinetosum sylvestris (Aichinger 1933) Br.-Bl. et Siss. 39) pro parte (T. Wraber 1979); Fraxino orni-Pinetum nigrae MartinBosse 1967 pinetosum sylvestris T. Wraber 1979 typus excluded (Poldini \& Vidali 1999); Fraxino orni-Pinetum nigrae pinetosum sylvestris T. Wraber 1979 var. Larix decidua Dakskobler 2006, typus included (Dakskobler 2006); Fraxino orni-Pinetum nigrae laricetosum deciduae Zupančič \& Žagar 2010, typus included, Fraxino orni-Pinetum nigrae caricetosum humilis Martin Bosse 1967, typus excluded pro parte (Zupančič \& Žagar 2010).

-euphorbietosum amygdaloidis subass. nov. hoc loco, nomenclatural type, holotypus, is relevé 17 in Table 1

-sorbetosum aucupariae subass. nov. hoc loco, nomenclatural type, holotypus, is relevé 28 in Table 1

\section{Genisto januensis-Pinetum sylvestris Tomažič 1940}

-campanuletum cespitosae Dakskobler in Rozman, Dakskobler et Šilc 2019 subass. nov. hoc loco, nomenclatural type, holotypus, is relevé 14 in Table 2.

\section{Ecological conditions in the studied pine stands}

Basophilic Scots pine forests from the association Rhodothamno chamaecisti-Pinetum sylvestris occur on predominantly dolomite bedrock at elevations ranging from 500 to $1500 \mathrm{~m}$, with the highest occurrence density at the elevations between 1000 and $1200 \mathrm{~m}$; stands from the association Genisto januensis-Pinetum sylvestris occur lower, at between 300 and $900 \mathrm{~m}$ a.s.l., and are the most frequent at elevations spanning 500 to $600 \mathrm{~m}$ (Figure 3). Mean annual temperature in the stands of the association Rhodothamno chamaecysti-Pinetum sylvestris is therefore lower (between 5 and $6^{\circ} \mathrm{C}$ ) than in the stands of the association Genisto januensis-Pinetum sylvestris (around 9 $\left.{ }^{\circ} \mathrm{C}\right)$. Stands of the association Genisto januensis-Pinetum sylvestris receive between 1800 and $2000 \mathrm{~mm}$ annual precipitation, whereas the annual precipitation in the stands of the association Rhodothamno chamaecysti-Pinetum sylvestris varies substantially due to geographical conditions. The eastern Karavanke Mts. receive less precipitation (1400-1600 mm) than the western Karavanke Mts. and the Julian Alps (1800-2000 mm). Monthly distribution of precipitation for the western part of the distribution area of associations Rhodothamno chamaecysti-Pinetum sylvestris and Genisto januensis-Pinetum sylvestris indicates that most of the precipitation is received in autumn with a small peak in early summer (Figure 4), whereas the autumn maximum is not observed in the eastern part of the distribution area and maximum precipitation is received in summer months.

Warm aspects predominate in both communities, with surface rockiness for the most part below 20\%. Stands of the association Genisto januensis-Pinetum sylvestris have a higher percentage of the tree layer cover, on average between 60 and $70 \%$, whereas the stands of the association Rhodothamno chamaecysti-Pinetum sylvestris have between 50 and $60 \%$. The herb layer cover in both communities exceeds $80 \%$ and in most part of the stands of the association Rhodothamno chamaecysti-Pinetum sylvestris the herb layer covers more than $90 \%$ of the forest floor. Trees are bigger in the stands of the association Rhodothamno chamaecysti-Pinetum sylvestris, with most trees measuring between 30 and $50 \mathrm{~cm}$ in diameter at breast height. Trees measuring more than $40 \mathrm{~cm}$ in diameter at breast height are very rare in the stands of the association Genisto januensis-Pinetum sylvestris. Trees are generally small, rarely taller than $20 \mathrm{~m}$.

Species diversity of the studied stands is mainly 40 to 60 species per relevé, slightly higher in the stands of the association Rhodothamno chamaecysti-Pinetum sylvestris, where the tree layer is less dense and the herb layer more compact. For the association Rhodothamno-Pinetum we also determined a higher total number of species (Figure 3).

Figure 3: Ecological conditions (Altitude, Northness, Stoniness, E3-tree layer coverage, E2-shrub layer coverage, E1-herb layer coverage, MeanTemp/Y-mean annual temperature, SumPrec/Y-annual sum of precipitation), some stand parameters ((Dmax-maximum tree diameter, Hmax-height of the tallest trees) and species diversity in studied Scots pine stands.

Slika 3: Ekološke razmere (nadmorska višina, severnost, kamnitost, E3-zastrtost drevesne plasti, E2-zastrtost grmovne plasti, E1-zastrtost zeliščne plasti, MeanTemp/Y-srednja letna temperatura, SumPrec/Y-letna količina padavin), nekateri sestojni parametri (Dmax-največji prsni premer, Hmax-višina najvišjih dreves) in vrstna pestrost v obravavanih sestojih rdečega bora.

Figure 4: Distribution of mean monthly temperature and precipitation sums in the stands of associations Genisto januensis-Pinetum sylvestris and Rhodothamno chamaecysti-Pinetum sylvestris; the stands of the latter are presented separately for the eastern and western part of the distribution area. Slika 4: Razpored povprečne mesečne temperature in padavinskih vsot v sestojih asociacij Genisto januensis-Pinetum sylvestris in Rhodothamno chamaecysti-Pinetum sylvestris; sestoji te združbe so prikazani ločeno za vzhodni in zahodni del areala. 

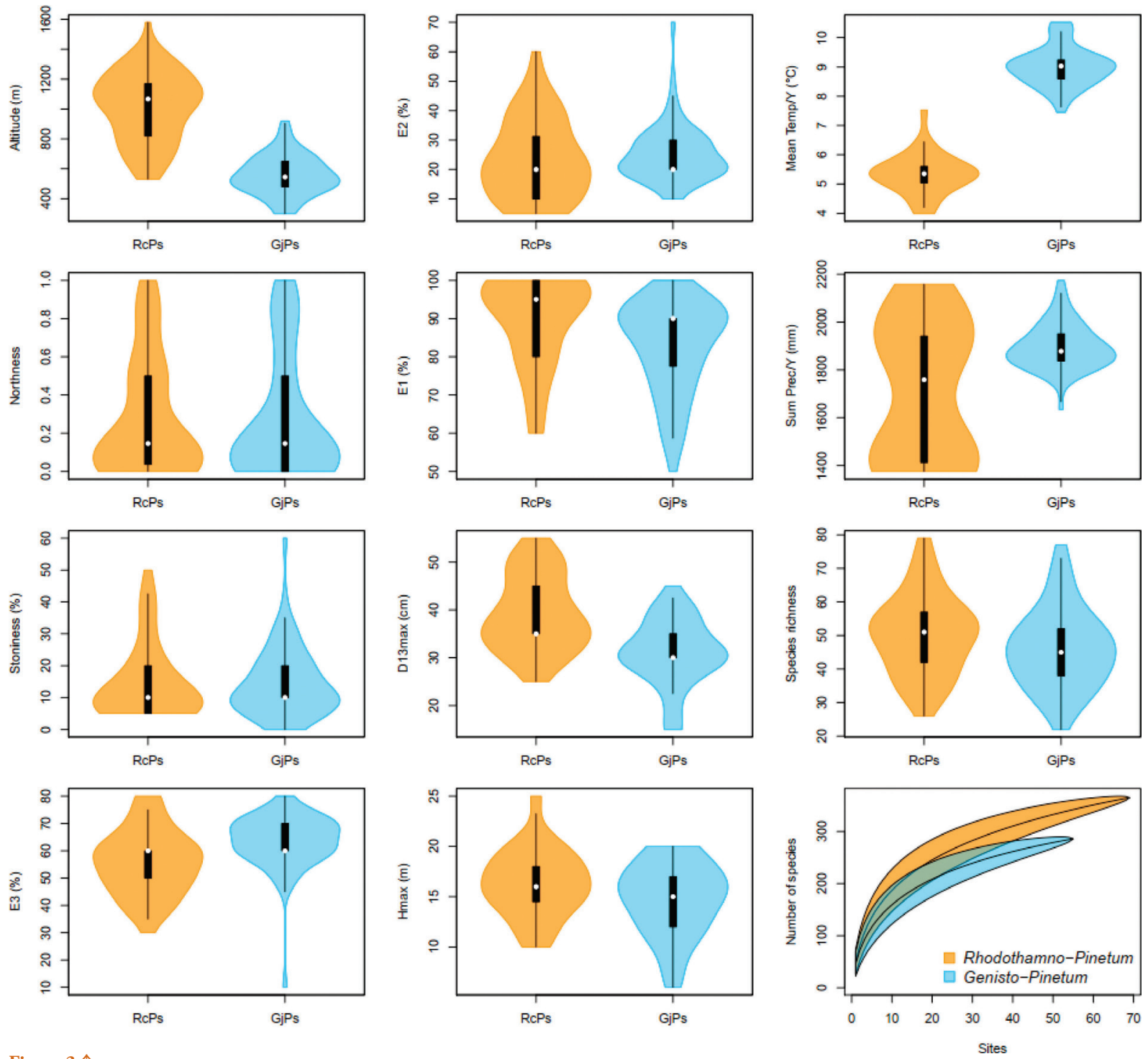

Figure $3 \uparrow$

Figure $4 \downarrow$
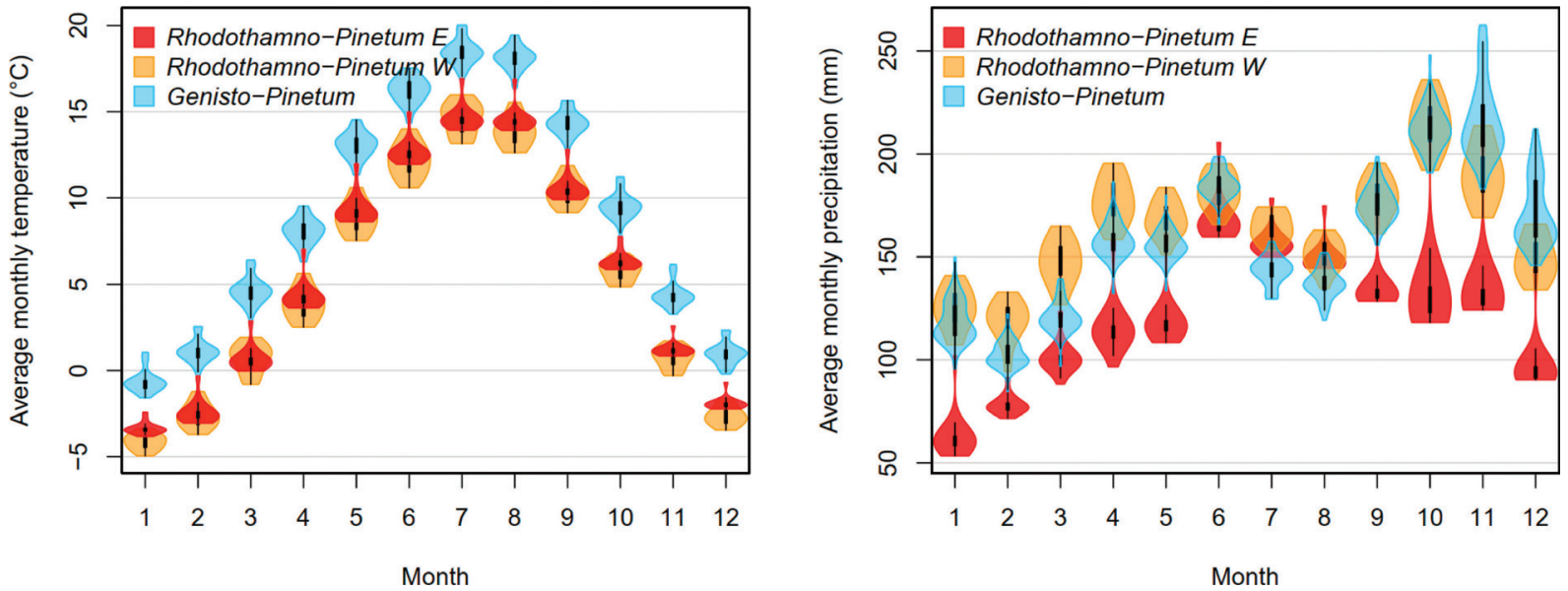

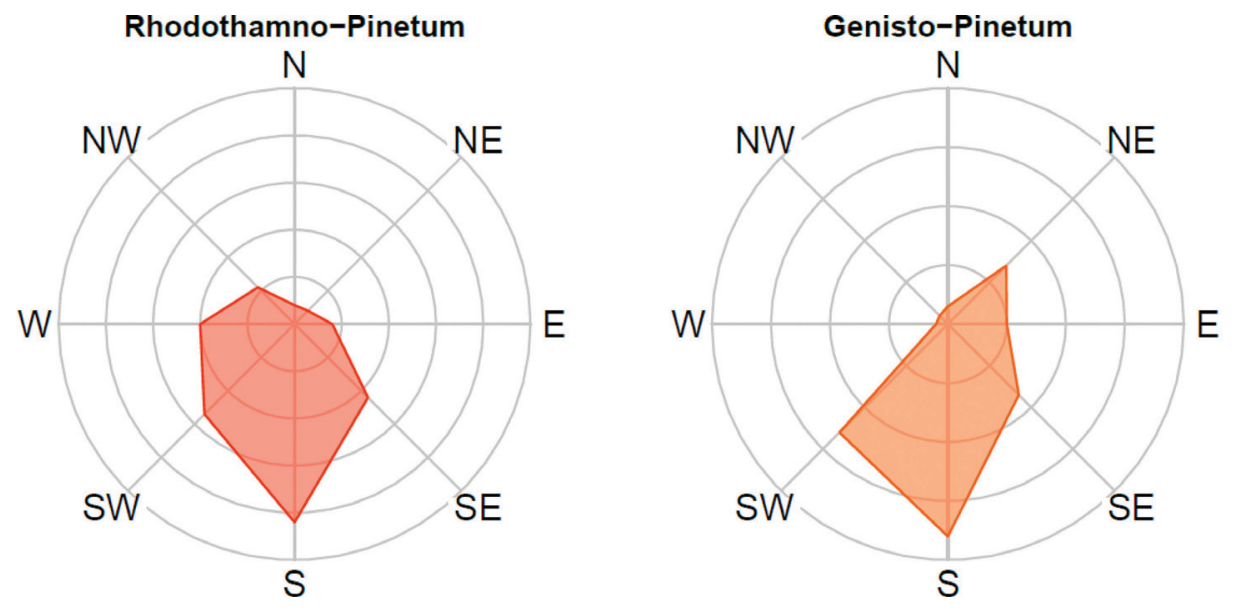

Figure 5: Rose diagrams of aspects showing mean slope in pine stands. Slika 5: Roži nebesnih leg s prikazom povprečne strmine borovih sestojev.

Sunny aspects on rather steep terrain prevail in both studied communities. The average slope of the stands of the association Rhodothamno chamaecysti-Pinetum sylvestris is about $40^{\circ}$, and about $\mathrm{v} 35^{\circ}$ in the stands of the association Genisto januensis-Pinetum sylvestris (Figure 5).

\section{Habitat typology and nature conservation status}

None of the FFH (Fauna-Flora-Habitat Directive) habitat types in the existing European Nature Information System (EUNIS) classification is quite appropriate for the Scots pine community on extreme sites in the Southeastern Alps. The list of threatened habitats in Austria (Essl et al. 2002) classifies the stands of the association Erico-Pinetum sylvestris as Scots pine forests on carbonate bedrocks (Karbonat-Rotföhrenwald) and does not classify them under any FFH habitat type. The studied community also cannot be classified into the habitat type (Sub)Mediterranean pine forests with endemic black pines $\left(9530^{*}\right)$. Proposed solution for the stands of the new association is to be classified into a new habitat subtype Southeastern-Alpine Scots pine forests of the Natura 2000 habitat type Southeastern European Scots pine forests (91R0). The distribution of basophilic Scots and black pine communities these pine communities in Slovenia is shown in Figure 6.

In terms of phytosociology the southeastern-Alpine Scots pine stands, despite their ecological and floristic similarities with black pine stands, should not be classified into the association Fraxino orni-Pinetum nigrae because of the absence of black pine. Numerical comparison of a large number of relevés provided enough reasons for them to be discussed separately in the syntaxonomic system. They could be classified into the Alpine association Erico-Pinetum sylvestris. Floristic similarity with stands of this association from the Dolomites is substantial, but considerably lower with the stands of this association in the Eastern Alps. Another option would be a new geographical variant. This rank is not discussed by the Code of Phytosociological Nomenclature. Describing a new association Rhodothamno-Pinetum sylvestris is therefore wellfounded; this association can comprise also ecologically similar Scots pine stands on similar sites in the Southern and Southeastern Alps in northern and northeast Italy, but not the stands of the subassociation Erico-Pinetum sylvestris ostryetosum carpinifolia from southern Austria. Despite a number of shared diagnostic species and the fact that the transition between the Alps and the Dinarides is smooth, without sharp divisions, the studied stands still cannot be classified into the Illyrian (northern-Dinaric) association Genisto-Pinetum sylvestris. The differences in the composition by groups of diagnostic species are too obvious. Differential species include above all species from the class Vaccinio-Piceetea, in particular larch (Larix decidua) and mountain cranberry (Vaccinium vitis-idaea). Stands of the association Rhodothamno-Pinetum sylvestris are classified also in the new forest site type SoutheasternAlpine basophilic Scots pine forests, whereas the existing forest site type Basophilic forests of Scots pine should be renamed as Pre-Alpine-Dinaric basophilic Scots pine forests (Kutnar et al. 2012: 204).

The stands of the The Southeastern-Alpine Scots pine community (Rhodothamno-Pinetum sylvestris) in Slovenia populates at least 496 ha (Šilc et al. 2017) as well as large surface areas in the western Julian Alps, the Carnic Alps and the Friuli Dolomites (Del Favero et al. 1998: 100). 


\begin{tabular}{lll}
\hline - Hacquetia $19 / 1 \bullet 2020,23-80$ & $\begin{array}{l}\text { Andrej Rozman, Igor Dakskobler \& Urban Šilc } \\
\text { Phytosociological analysis of basophilic Scots pine forests in the } \\
\text { Southeastern Alps }\end{array}$ \\
\hline
\end{tabular}

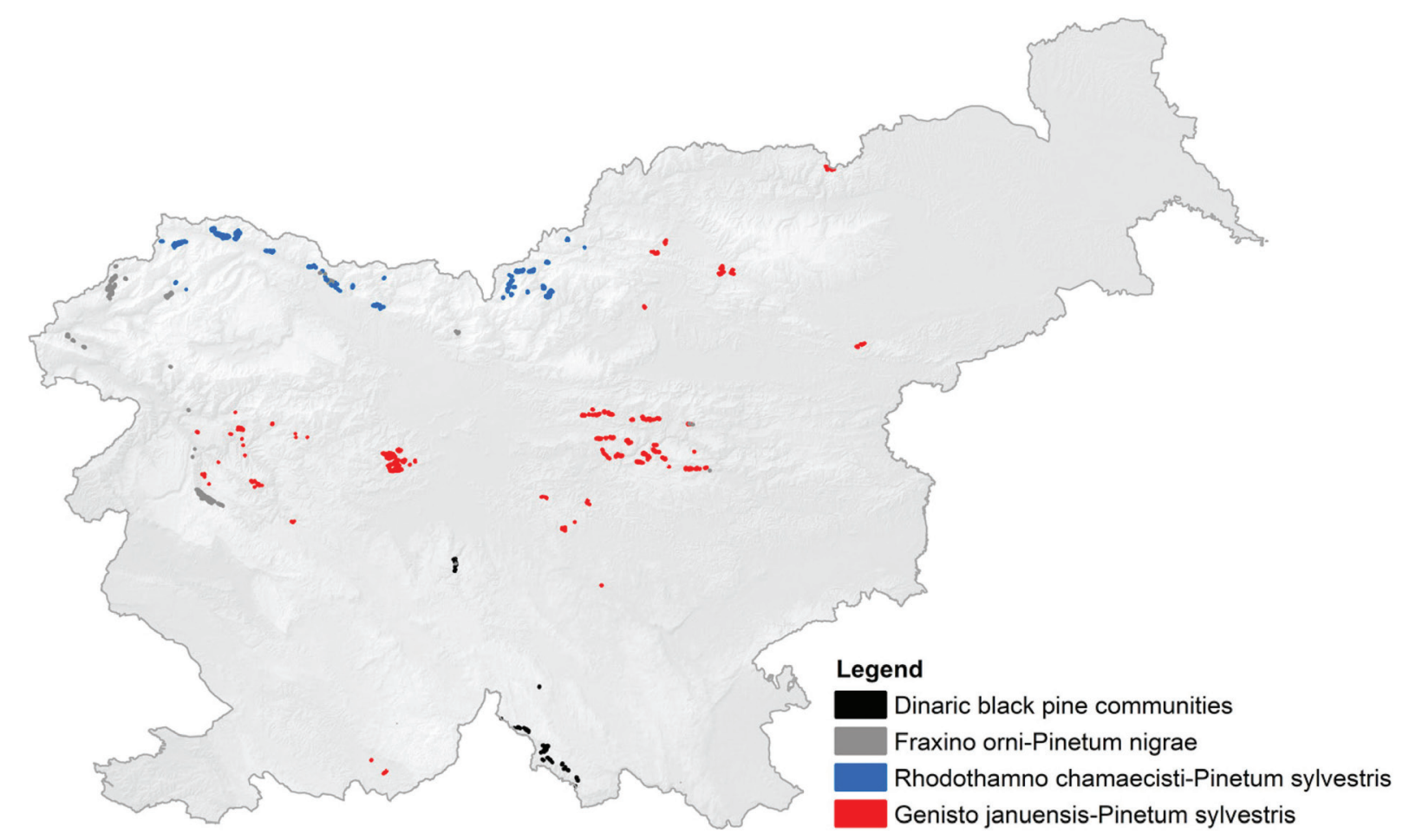

Figure 6: Distribution map of basophilic Scots and black pine communities in Slovenia.

Slika 6: Karta razširjenosti obravnavanih združb rdečega in črnega bora v Sloveniji.

These are mainly protective forests and their commercial value is therefore negligible. In Slovenia, they are a constituent part of the following forest reserves: Kukla, Mala Pišnica, Belca, Matkov kot-Logarska dolina (Mlinšek et al. 1980, Marenče 2003), as well as one of the forest types in the Triglav National Park, the Logarska dolina (Logar Valley) Landscape Park and the Topla Landscape Park. Southeastern-Alpine Pinus sylvestris forest species comprise also Red list species (Anon. 2002) Orobanche teucrii and several protected species (Anon. 2004): Arctostaphylos uva-ursi, Cephalanthera rubra, C. damasonium, C. longifolia, Convallaria majalis, Cyclamen purpurascens, Daphne cneorum, Dactylorhiza fuchsii, Dianthus sylvestris, Diphasiastrum complanatum, Epipactis atrorubens, E. helleborine, E. muelleri, Gentiana clusii, Goodyera repens, Gymnadenia odoratissima, G. conopsea, Helleborus niger, Huperzia selago, Iris graminea, Lilium martagon, Listera cordata, L. ovata, Neottia nidus-avis, Ophrys insectifera, Pinguicula alpina, Platanthera bifolia, Primula auricula, and Taxus baccata.

In the stands of the subassociation Genisto januensiPinetum sylvestris campanuletosum cespitosae we listed also Natura 2000 plant species Primula carniolica, Red List species (Anon. 2002): Hemerocallis lilioasphodelus, Schoenus nigricans and Veratrum nigrum, and the following protected species (Anon. 2004): Cephalanthera rubra,
C.damasonium, C. longifolia, Convallaria majalis, Cyclamen purpurascens, Dianthus hyssopifolius (D. monspessulanus), Epipactis atrorubens, E. helleborine, E. muelleri, Gymnadenia conopsea, G. odoratissima, Helleborus niger, Ilex aquifolium, Iris graminea, Lilium carniolicum, L. martagon, Listera ovata, Neottia nidus-avis, Ophrys insectifera, Pinguicula alpina, Platanthera bifolia, and Taxus baccata.

\section{Povzetek}

Fitocenološka oznaka naravnih bazoljubnih sestojev rdečega bora (Pinus sylvestris) v Jugovzhodnih Alpah

$S$ fitocenološko obdelavo obsežnega popisnega gradiva bazoljubnih združb rdečega in črnega bora v Južnih, Jugovzhodnih in Vzhodnih Alpah (spodbudila jih je aplikativna raziskava, Šilc et al. 2017), smo želeli preveriti, ali je smiselno $\mathrm{v}$ glavnem čiste sestoje rdečega bora, ki rastejo ponekod v Julijskih in Kamniško-Savinjskih Alpah ter v Karavankah, razlikovati od v glavnem čistih sestojev črnega bora na podobnih rastiščih v Julijskih Alpah in zahodnih Karavankah, zelo redko v Kamniško-Savinjskih Alpah (dolina Kokre). Z analizo zbranega popisnega gradiva smo ugotovili, da združbo rdečega bora na skrajnih rastiščih v Jugovzhodnih Alpah nikakor ne moremo uvrstiti v habitatni tip (Sub)mediteranski gozdovi črnega 
bora $\left(9530^{*}\right)$, prav tako ni v obstoječi Eunis klasifikaciji habitatnih tipov zanjo noben FFH habitatni tip povsem ustrezen. V seznamu ogroženih habitatov Avstrije (Essl et al. 2002) sestoje asociacije Erico-Pinetum sylvestris uvrščajo $\mathrm{k}$ združbam rdečega bora na karbonatni podlagi (Karbonat-Rotföhrenwald), vendar ti ne sodijo v noben FFH habitatni tip. Sestoje nove asociacije zato za zdaj uvrščamo v Natura 2000 habitatni tip Jugovzhodnoevropski gozdovi rdečega bora (91R0) in predlagamo poseben habitatni podtip Jugovzhodnoalpski gozdovi rdečega bora.

$\mathrm{V}$ fitocenološkem smislu jugovzhodnoalpske sestoje rdečega bora, kljub ekološkim in florističnim podobnostim s sestoji črnega bora zaradi odsotnosti le tega, ni ustrezno uvrščati v asociacijo Fraxino orni-Pinetum nigrae. Numerična primerjava velikega števila popisov daje dovolj razlogov, da jih v sintaksonomskem sistemu obravnavamo ločeno. Njihova uvrstitev $\mathrm{v}$ alpsko asociacijo Erico-Pinetum sylvestris je mogoča. Floristična podobnost $s$ sestoji te asociacije iz Dolomitov je precejšnja, bistveno manjša pa s sestoji te asociacije v Vzhodnih Alpah. Mogoči rang bi bila nova geografska varianta. Tega ranga Kodeks fitocenološke nomenklature ne obravnava. Zato je utemeljen opis nove asociacije Rhodothamno-Pinetum sylvestris, v katero lahko uvrstimo tudi ekološko in rastiščno podobne sestoje rdečega bora iz Južnih in Jugovzhodnih Alp v severni in severovzhodni Italiji, ne pa sestojev subasociacije Erico-Pinetum sylvestris ostryetosum carpinifolia iz južne Avstrije. Priključitev preučenih sestojev ilirski (severno-dinarski) asociaciji Genisto-Pinetum sylvestris je kljub precej skupnim diagnostičnim vrstam in dejstvu, da je prehod med Alpami in Dinarskim gorstvom zelo zvezen, brez ostrih meja, ni mogoča. Razlike v sestavi po skupinah diagnostičnih vrst so preveč očitne. Razlikovalne so predvsem vrste razreda Vaccinio-Piceetea, še posebej macesen (Larix decidua) in brusnica (Vaccinium vitisidaea). Sestoje asociacije Rhodothamno-Pinetum sylvestris uvrščamo tudi v nov gozdni rastiščni tip Jugovzhodnoalpsko bazoljubno rdečeborovje, medtem ko je obstoječi gozdni rastiščni tip Bazoljubno rdečeborovje treba preimenovati v Predalpsko-dinarsko bazoljubno rdečeborovje (Kutnar et al. 2012: 204).

Jugovzhodnoalpski sestoji rdečega bora (RhodothamnoPinetum sylvestris) v Sloveniji poraščajo najmanj 496 ha (Šilc et. al.2017), prav tako pa se pojavljajo še na obsežnih površinah zahodnih Julijskih Alp, Karnijskih Alp in v Dolomitih (Del Favero et al. 1998: 100). Večinoma gre za varovalne gozdove $\mathrm{z}$ zanemarljivim gozdnogospodarskim pomenom. Ti gozdovi so v Sloveniji sestavni del vegetacije večih gozdnih rezervatov: Kukla, Mala Pišnica, Belca, Matkov kot-Logarska dolina (Mlinšek et al. 1980, Marenče 2003); in naravnih parkov: Triglavski narodni park, krajinski park Logarska dolina, krajinski park Topla.
V Jugovzhodnoalpskih gozdovih rdečega bora raste tudi vrsta $z$ rdečega seznama ogroženih rastlinskih vrst (Anon. 2002) Orobanche teucrii in številne druge zavarovane vrste (Anon. 2004): Arctostaphylos uva-ursi, Cephalanthera rubra, C. damasonium, C. longifolia, Convallaria majalis, Cyclamen purpurascens, Daphne cneorum, Dactylorhiza fuchsii, Dianthus sylvestris, Diphasiastrum complanatum, Epipactis atrorubens, E. helleborine, E. muelleri, Gentiana clusii, Goodyera repens, Gymnadenia odoratissima, G. conopsea, Helleborus niger, Huperzia selago, Iris graminea, Lilium martagon, Listera cordata, L. ovata, Neottia nidusavis, Ophrys insectifera, Pinguicula alpina, Platanthera bifolia, Primula auricula in Taxus baccata.

$\mathrm{V}$ Cerkljanskem in Idrijskem hribovju in deloima $\mathrm{v}$ dolini Trebuše (Pršjak) smo opisali novo subasociacijo severno-dinarskega (ilirskega) bazoljubnega rdečega borovja Genisto januensi-Pinetum sylvestris campanuletosum cespitosae, kjer so rastišča Natura 2000 vrste Primula carniolica, vrst rdečega seznama ogroženih rastlinskih vrst (Anon. 2002): Hemerocallis lilioasphodelus, Schoenus nigricans in Veratrum nigrum in zavarovanih vrst (Anon. 2004): Cephalanthera rubra, C. damasonium, C. longifolia, Convallaria majalis, Cyclamen purpurascens Dianthus hyssopifolius (D. monspessulanus), Epipactis atrorubens, E. helleborine, E. muelleri, Gymnadenia conopsea, G. odoratissima, Helleborus niger, Ilex aquifolium, Iris graminea, Lilium carniolicum, L. martagon, Listera ovata, Neottia nidus-avis, Ophrys insectifera, Pinguicula alpina, Platanthera bifolia in Taxus baccata.

\section{Acknowledgements}

The research was conducted in the framework of the target research programmeThe design of monitoring of the conservation status of minor Natura 2000 forest habitat types in Slovenia (V4-1430) funded by the Slovenian Research Agency and Ministry of Agriculture, Forestry and Food. We acknowledge also the financial support from the Slovenian Research Agency (research core funding No. P1-0236) and from the foundation Pahernikova ustanova. Sincere thanks to Prof. Dr. Jean-Paul Theurillat for his valuable advice in the description of the new association, and to Dr. Mateja Cojzer, Žiga Repotočnik and Dr. Branko Vreš for their help in the field work. Two anonymous reviewers helped us with valuable improvements and corrections. English translation by Andreja Šalamon Verbič.

Andrej Rozman (D), https://orcid.org/0000-0003-0797-5452 Urban Šilc DD, https://orcid.org/0000-0002-3052-699X 


\section{References}

Accetto, M. 1999: Asociacija Carici sempervirentis-Pinetum nigrae (Accetto 1996) Accetto 1999 nom. nov. v Sloveniji (ob stoletnici rojstva prvega slovenskega fitocenologa univ. prof. Gabrijela Tomažiča). Zbornik gozdarstva in lesarstva 60: 197-151.

Accetto, M. 2001: Asociacija Daphno alpinae-Pinetum nigrae ass. nova v Sloveniji. Zbornik gozdarstva in lesarstva 64: 5-39.

Accetto, M. 2008: Floristične in vegetacijske zanimivosti z ostenij na severnih, severozahodnih in zahodnih pobočjih doline potoka Prušnice (0152/1, del). Razprave 4. razreda SAZU 49-1: 5-53.

Accetto, M. 2010: Rastlinstvo Iškega Vintgarja. Praprotnice in semenke. Folia biologica et geologica 51 (4): 5-149.

Accetto, M. 2013: Rastlinstvo in deloma rastje soteske Zale v zgornjem porečju Iške. Zbornik gozdarstva in lesarstva 99: 3-149.

Accetto M. 2015: Gozdno in drugo rastje na levem bregu Iškega vintgarja. Forest and other plant communities on the Iški vintgar left bank. Acta silvae et ligni 106: 1-121.

Aeschimann, D., Lauber, K., Moser, D. M. \& Theurillat, J.-P. 2004a: Flora alpina. Bd. 1: Lycopodiaceae-Apiaceae. Haupt Verlag, Bern, Stuttgart, Wien, 1159 pp.

Aeschimann, D., Lauber, K., Moser, D. M. \& Theurillat, J.-P. 2004b: Flora alpina. Bd. 2: Gentianaceae-Orchidaceae. Haupt Verlag, Bern, Stuttgart, Wien, 1188 pp.

Aichinger, E. 1933: Vegetationskunde der Karawanken. Gustav Fischer Verlag, Jena 329 pp.

Anonymous 2002: Pravilnik o uvrstitvi ogroženih rastlinskih in živalskih vrst v rdeči seznam. Uradni list RS 82/2002.

Anonymous 2004: Uredba o zavarovanih prosto živečih rastlinskih vrstah. Uradni list RS 46/2004.

Braun-Blanquet, J. 1964: Pflanzensoziologie. Grundzüge der Vegetationskunde. 3. Auflage. Springer, Wien - New York, 865 pp.

Buser, S. 2009: Geološka karta Slovenije 1: 250.000. Geological map of Slovenia 1.250,000. Geološki zavod Slovenije, Ljubljana.

Cimperšek, M. 2005: Varovalni gozdovi rdečega bora (Genisto januensisPinetum sylvestris) in puhastega hrasta ter črnega gabra (Querco-Ostryetum carpinifoliae) na Boču. Gozdarski vestnik 63 (5-6): 235-252.

Dakskobler, I. 1998a: Vegetacija gozdnega rezervata Govci na severovzhodnem robu Trnovskega gozda (zahodna Slovenija). In: J. Diaci (ed.): Gorski gozd. Zbornik referatov. 19. gozdarski študijski dnevi, Logarska dolina 26. - 27. 3. 1998, Ljubljana, pp. 269-301

Dakskobler, I. 1998b: Naravni sestoji črnega bora (Pinus nigra Arnold) na vzpetini Treska pri Srpenici in nad dolino Tolminke (Julijske Alpe, severozahodna Slovenija). Razprave 4. razreda SAZU 39 (7): 255-278.

Dakskobler, I. 1999: Contribution to the knowledge of the association Fraxino orni-Pinetum nigrae Martin-Bosse 1967. Wissenschaftliche Mitt. Niederösterr. Landesmuseum 12: 25-52.

Dakskobler, I. 2006: The Association Rhodothamno-Laricetum (Zukrigl 1973) Willner \& Zukrigl 1999 in the Julian Alps. Razprave 4. razreda SAZU 47-1: 117-192.
Dakskobler, I., Kutnar, L., Rozman, A. 2015: Bazoljubno borovje v Sloveniji. Združbe črnega in rdečega bora na karbonatni podlagi in rušja v alpskih dolinah. Studia forestalia Slovenica 144. Silva Slovenica, Gozdarski inštitut Slovenije, Ljubljana, 120 pp.

De Caceres M., \& Legendre P. 2009: Associations between species and groups of sites: indices and statistical inference. Ecology 90 (12): 3566-3574

Del Favero, R., Poldini, L., Bortoli, P., Dreossi, G., Lasen, C. \& Vanone, G. 1998: La vegetazione forestale e la selvicoltura nelle regione Friuli - Venezia Giulia. Vol. 2. Regione autonoma Friuli-Venezia Giulia. Direzione Regionale delle Foreste, Servizio delle Selvicoltura, Udine, 303 pp. + Appendix.

Dufrene, M. \& Legendre, P. 1997: Species assemblages and indicator species: the need for a flexible asymmetrical approach. Ecol. Monogr. 67(3): 345-366.

Eichberger, C., Heiselmayer, P. \& Grabner, S. 2004: Rotföhrenwälder in Osterreich: eine syntaxonomische Neubewertung. Tuexenia 24: $127-176$.

Eichberger, P., Heiselmayer, P. \& Grabner, S. 2007 a: Erico-Pinion sylvestris Br.-Bl. 1939. In: Willner, W. \& Grabherr, G. (eds.): Die Wälder und Gebüsche Österreichs. Ein Bestimmungswerk mit Tabellen. 1. Textband. Spektrum Akademischer Verlag in Elsevier, Heidelberg, pp. 169-174.

Eichberger, P., Heiselmayer, P. \& Grabner, S. 2007 b: Erico-Pinetum sylvestris. In: Willner, W. \& Grabherr, G. (eds.): Die Wälder und Gebüsche Österreichs. Ein Bestimmungswerk mit Tabellen. 2. Tebellenband. Spektrum Akademischer Verlag in Elsevier, Heidelberg, pp. 190-194.

Essl, F., Egger, G., Ellmauer, T. \& Aigner, S. 2002: Rote liste gefährdeter biotoptypen Österreichs. Wälder, Forste, Vorwälder. Umweltbundesamt GmbH, 104 pp.

Franz, W. R. 2002: Die Hopfenbuche (Ostrya carpinifolia Scop.) in Österreich und Nord-Slowenien. Naturwissenschaftliche Verein für Kärnten, Klagenfurt, 256 pp.

Jennings, M. D., Faber-Langendoen, D., Loucks, O. L., Peet, R. K. \& Roberts, D. 2009: Standards for associations and alliances of the U. S. National Vegetation Classification. Ecol. Monogr. 79 (2): 173-199.

Kutnar, L., Veselič, Ž., Dakskobler I. \& Robič, D. 2012: Tipologija gozdnih rastišč Slovenije na podlagi ekoloških in vegetacijskih razmer za potrebe usmerjanja razvoja gozdov. Gozdarski vestnik 70 (4): $195-214$.

Landolt, E., Bäumler, B. Erhardt, A., Hegg, O., Klötzli, F., Lämmler, W., Nobis, M., Rudmann-Maurer, K., Schweingruber, F. H. , Theurillat, J.-P., Urmi, E., Vust, M. \& Wohlgemuth, T. 2010: Flora indicativa. 2. Auflage. Haupt Verlag, Bern-Stuttgart-Wien, 323 pp.

Maarel, van der E. 1979: Transformation of cover-abundance values in phytosociology and its effects on community similarity. Vegetatio 39 (2): 97-114.

Maarel, van der E. \& Franklin, J. 2013: Vegetation Ecology. 2nd Edition. Wiley-Blackwell. 572 pp.

Marenče, M. 2003: Gozdni rezervati v Triglavskem narodnem parku. Triglavski razgledi 7 (11): 1-24.

Martin-Bosse, H. 1967: Schwarzföhrenwälder in Kärnten. Angewandte Pflanzensoziologie 20: 1-97, Springer, Wien - New York. 
Martinčič, A. 2003: Seznam listnatih mahov (Bryopsida) Slovenije. Hacquetia 2 (1): 91-166.

Martinčič, A. 2011: Annotated Checklist of Slovenian Liverworts (Marchanthiophyta) and Hornworts (Anthocerotophyta). Scopolia 72: $1-38$.

Martinčič, A., Wraber, T., Jogan, N., Podobnik, A., Turk, B., Vreš, B., Ravnik, V., Frajman, B., Strgulc Krajšek, S., Trčak, B., Bačič, T., Fischer, M. A., Eler, K. \& Surina, B. 2007: Mala flora Slovenije. Ključ za določanje praprotnic in semenk. Četrta, dopolnjena in spremenjena izdaja. Tehniška založba Slovenije, Ljubljana, 967 pp.

Mlinšek, D., Accetto, M., Anko, B., Piskernik, M. Robič, D., Smolej, I. \& Zupančič, M. 1980: Gozdni rezervati v Sloveniji. Inštitut za gozdno in lesno gospodarstvo pri Biotehniški fakulteti v Ljubljani, Ljubljana, $414 \mathrm{pp}$.

Mucina, L., Bultmann, H., Dierssen, K., Theurillat, J. P., Raus, T., Carni, A., Sumberova, K., Willner, W., Dengler, J., Garcia, R. G., Chytry, M., Hajek, M., Di Pietro, R., Iakushenko, D., Pallas, J., Daniels, F. J. A., Bergmeier, E., Santos Guerra, A., Ermakov, N., Valachovic, M., Schaminee, J. H. J., Lysenko, T., Didukh, Y. P., Pignatti, S., Rodwell, J. S., Capelo, J., Weber, H. E., Solomeshch, A., Dimopoulos, P., Aguiar, C., Hennekens, S. M. \& Tichy, L. 2016: Vegetation of Europe: hierarchical floristic classification system of vascular plant, bryophyte, lichen, and algal communities. Applied Vegetation Science 19: 3-264.

Oksanen, J., Blanchet, F. G., Friendly, M., Kindt, R., Legendre, P., McGlinn, D., Minchin, P. R., O’Hara, R. B., Simpson, G. L., Solymos, P., Stevens, M. H. H., Szoecs, E. \& Wagner, H. 2018: vegan: Community Ecology Package. R package version 2.5-2. https:// CRAN.R-project.org/package $=$ vegan

Pignatti, S., 2005: Valori di bioindicazione delle piante vascolari della flora d'Italia: Bioindicator values of vascular plants of the Flora of Italy. Braun-Blanquetia 39: 96pp.

Pignatti, E. \& Pignatti, S. 2014: Plant Life of the Dolomites. Vegetation Structure and Ecology. Publication of the Museum of Nature South Tyrol Nr. 8, Naturmuseum Südtirol, Bozen, Springer Verlag, Heidelberg, 769 pp.

Pignatti, E. \& Pignatti, S. 2016: Plant Life of the Dolomites. Vegetation Tables. Publication of the Museum of Nature South Tyrol Nr. 11, Bozen, Springer Verlag, Heidelberg, 575 pp.

Piskernik, M. 1979: Vegetacija gozdov rdečega in črnega bora na slovenskem ozemlju. Zbornik gozdarstva in lesarstva 17 (2): 393-448.

Podani, J. 2001: SYN-TAX 2000. Computer Programs for Data Analysis in Ecology and Systematics. User's Manual, Budapest, 53 pp.

Poldini, L. 1969: Le pinete di pino austriaco nelle Alpi Carniche. Boll. Soc. Adr. Sci. Nat. 57: 3-65.

Poldini, L. 1982: Ostrya carpinifolia-Reiche Wälder und Gebüsche von Friaul-Julisch-Venezien (NO-Italien) und Nachbargebieten. Studia Geobotanica 2: 69-122.

Poldini, L. \& Vidali, M. 1999: Kombinationsspiele unter Schwarzföhre, Weißkiefer, Hopfenbuche und Mannaesche in den Südostalpen - Wiss. Mitt. Niederösterr. Landesmuseum (St. Pölten) 12: 105-136.
R Core Team 2018: R: A language and environment for statistical computing. R Foundation for Statistical Computing, Vienna, Austria. URL https://www.R-project.org/.

Seliškar, T., Vreš, B. \& Seliškar, A. 2003: FloVegSi 2.0. Fauna, Flora, Vegetation and Paleovegetation of Slovenia. Computer programme for arranging and analysis of biological data. Biološki inštitut ZRC SAZU, Ljubljana.

Schmid, E. 1936: Die Reliktföhrenwälder der Alpen. Beiträge zur geobotanischen Landesaufnahme der Schweiz 21, Bern, 190 pp.

Sørensen, Th. 1948: A method of establishing groups of equal amplitude in plant sociology based on similarity of species content. Det Kongelige Danske Videnskaberns Selskab, Biologiske Skrifter 5 (4): $1-34$.

Suppan, U., Prügger, J. \& Mayrhofer, H. 2000: Catalogue of the lichenized and lichenicolous fungi of Slovenia. Bibliotheca Lichenologica 76: 1-215.

Šilc, U. 2012: Vegetation database of Slovenia. Biodiversity \& Ecology 4: 428 .

Šilc, U. \& Čarni, A. 2012: Conspectus of vegetation syntaxa in Slovenia. Hacquetia 11 (1): 113-164.

Šilc, U., Čarni, A., Dakskobler, I., Kutnar, L. Marinšek, A. Rozman, A. Sajko, I. \& Vreš, B. 2017: Zasnova monitoringa stanja ohranjenosti manjšinskih Natura 2000 gozdnih habitatnih tipov v Sloveniji. Zaključno poročilo v okviru Ciljnega raziskovalnega projekta (V41430). Biološki inštitut ZRC SAZU, Ljubljana, 170 pp.

Theurillat, J.-P. 2004: Pflanzensoziologisches System. In: Aeschimann, D., Lauber, K., Moser D. M. \& Theurillat J.-P.: Flora alpina 3: Register. Haupt Verlag, Bern, Stuttgart, Wien, pp. 301-313.

Tomažič, G. 1940: Asociacije borovih gozdov v Sloveniji. I. Bazifilni borovi gozdi. Razprave matematično-prirodoslovnega razreda Akademije znanosti in umetnosti 1: 77-120.

Urbančič, M., Simončič, P., Prus, T. \& Kutnar, L. 2005: Atlas gozdnih tal. Zveza gozdarskih društev Slovenije, Gozdarski vestnik \& Gozdarski inštitut Slovenije, Ljubljana. 100 pp.

Weber, H. E., Moravec, J. \& Theurillat, J. P. 2000: International Code of Phytosociological Nomenclature. 3rd Edition. Journal of Vegetation Science 11 (5): 739-766.

Wraber, M. 1960: Fitocenološka razčlenitev gozdne vegetacije v Sloveniji. Zbornik ob 150. letnici botaničnega vrta v Ljubljani, Ljubljana, pp. 49-94.

Wraber, T. 1979: Die Schwarzföhrenvegetation des Koritnica Tales (Julische Alpen). Biološki vestnik 27(2): 199-204.

Zupančič, M. \& Žagar, V. 2010: Association Fraxino orni-Pinetum nigrae Martin-Bosse 1967 in the south-eastern Alpine Region. Folia biologica et geologica 51 (4): 177-225. 
Appendix 1: An overview of analytical tables, included in the analysis of pine stands.

Priloga 1: Pregled analitskih tabel, ki smo jih vključili v analizo borovih sestojev.

\begin{tabular}{lllll}
\hline Sign / & No. Rel. / & Association / & Author / & Mountain range / \\
Oznaka & St. pop. & Asociacija & Avtor & Pogorje \\
\hline EcPs.Pa.15 & 20 & Erico-Pinetum sylvestris & Pignatti & Dolomites \\
FoPn.Db.2 & 4 & Fraxino orni-Pinetum nigrae & Dakskobler & Julian Alps \\
FoPn.Dc.3 & 22 & Fraxino orni-Pinetum nigrae & Dakskobler & Julian Alps \\
FoPn.Dd.4 & 30 & Fraxino orni-Pinetum nigrae & Dakskobler & Trnovski Gozd \\
FoPn.Df.6 & 5 & Fraxino orni-Pinetum nigrae & Dakskobler & Julian Alps \\
FoPn.Gf.Dg.7 & 10 & Fraxino orni-Pinetum nigrae & Dakskobler & Kamnik Alps \\
FoPn.Ra.9 & 13 & Fraxino orni-Pinetum nigrae & Rozman & W Karavanke \\
FoPn.pn.Wa.13 & 12 & Fraxino orni-Pinetum nigrae & Wraber & Julian Alps \\
FoPn.pn.PVa.16 & 17 & Fraxino orni-Pinetum nigrae & Poldini_Vidali & Julian and Carnic Alps \\
FoPn.ch.MBa.18 & 28 & Fraxino orni-Pinetum nigrae & Martin-Bose & A Carinthia \\
FoPn.cv.MBb.19 & 16 & Fraxino orni-Pinetum nigrae & Martin-Bose & A Carinthia \\
FoPn.cv.Pp.MBc.20 & 9 & Fraxino orni-Pinetum nigrae & Martin-Bose & A Carinthia \\
GjPs.Di.21 & 55 & Genisto-Pinetum & Dakskobler & Cerkljansko Hills \\
RcPs.ld.Da.1 & 8 & Rhodothamno chamaecisti-Pinetum sylvestris & Dakskobler & Julijske Alpe \\
RcPs.De.5 & 4 & Rhodothamno chamaecisti-Pinetum sylvestris & Dakskobler & Julian pre-Alps \\
RcPs.Dh.8 & 15 & Rhodothamno chamaecisti-Pinetum sylvestris & Dakskobler & E Karavanke \\
RcPs.Rb.10 & 13 & Rhodothamno chamaecisti-Pinetum sylvestris & Rozman & W Karavanke \\
RcPs.ld.Z.11 & 4 & Rhodothamno chamaecisti-Pinetum sylvestris & Zupančič & Kamnik Alps \\
RcPs.ch.Z.12 & 7 & Rhodothamno chamaecisti-Pinetum sylvestris & Zupančič & Julian Alps, W Karavanke \\
RcPs.Wb.14 & 5 & Rhodothamno chamaecisti-Pinetum sylvestris & Wraber & Julian Alps, W Karavanke \\
RcPs.PVa.17 & 11 & Rhodothamno chamaecisti-Pinetum sylvestris & Poldini_Vidali & Julian and Carnic Alps \\
\hline & & & &
\end{tabular}

Appendix 2: Distribution of researched stands of the subassociation Genisto januensis-Pinetum sylvestris campanuletosum cespitosae on the map of Slovenia.

Priloga 2: Razširjenost preučenih sestojev subasociacije Genisto januensis-Pinetum sylvestris campanuletosum cespitosae na zemljevidu Slovenije.

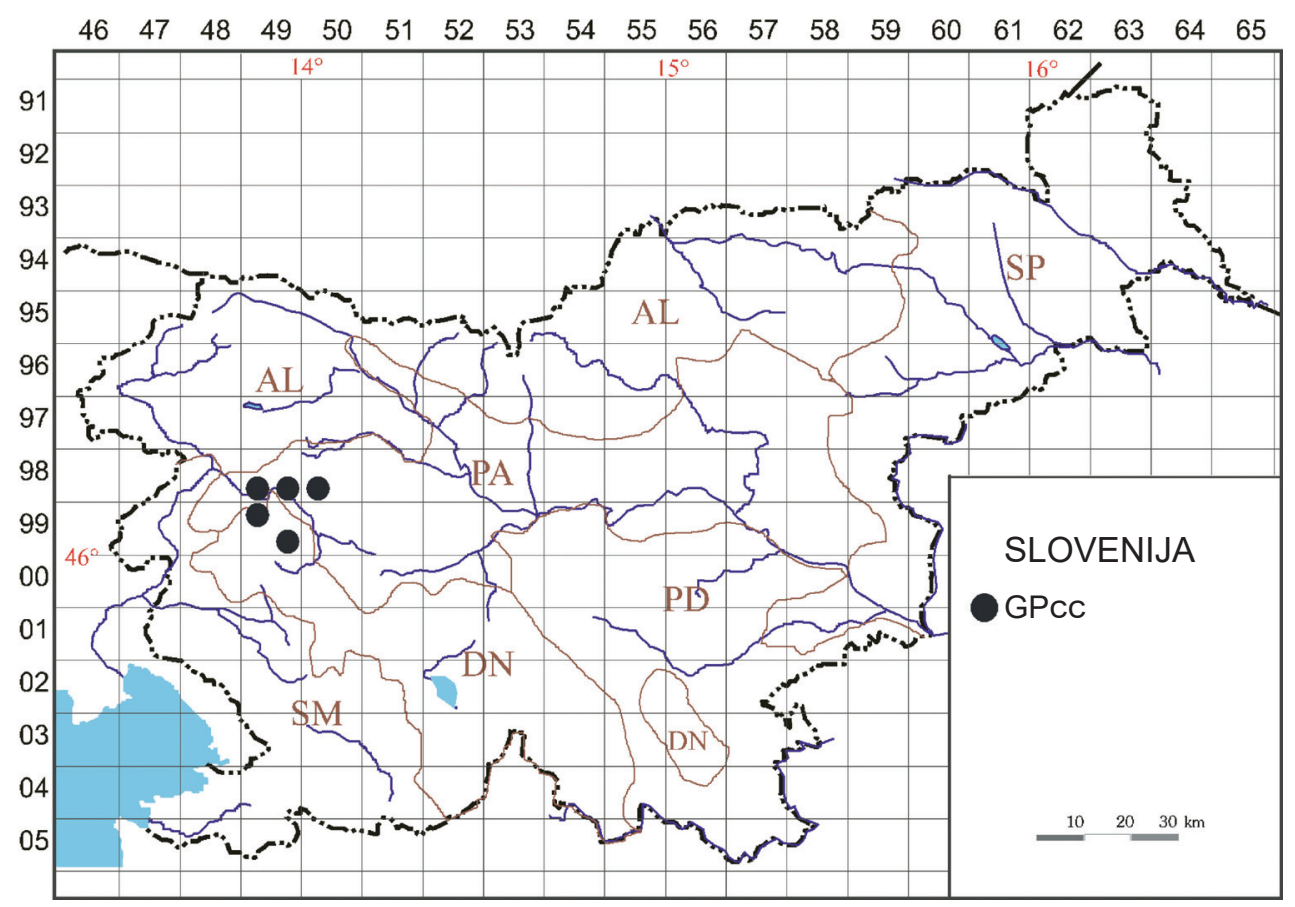


Table 1 (Tabela 1): Rhodothamno chamaecisti-Pinetum sylvestris ass. nov.

\section{Number of relevé (Zaporedna štev. popisa)}

Database number of relevé

(Delovna številka popisa)

Author of the relevé

(Avtor popisa)

Elevation in $\mathrm{m}$

(Nadmorska višina $\mathrm{v} \mathrm{m}$ )

Aspect (Lega)

Slope in degrees (Nagib v stopinjah)

Parent material (Matična podlaga)

Soil (Tla)

Stoniness in \% (Kamnitost v \%)

Cover in \% (Zastiranje v \%):

Tree layer (drevesna plast)

Shrub layer (Grmovna plast)

Herb layer (Zeliščna plast)

Moss layer (Mahovna plast)

Number of species (Število vrst)

Relevé area (Velikost popisne ploskve)

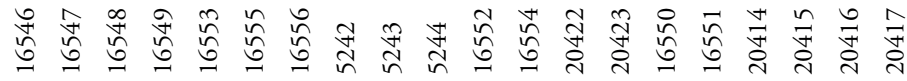

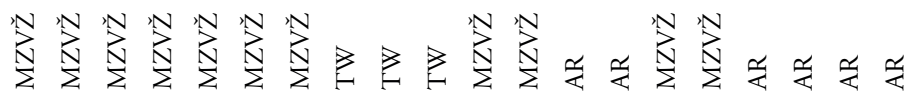

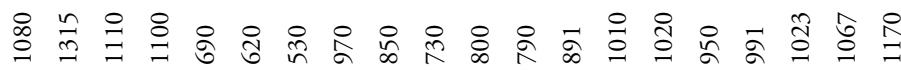

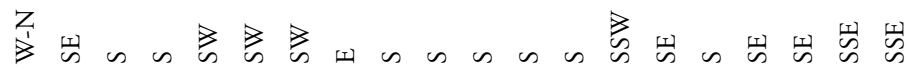
$70 \begin{array}{lllllllllllllllllll}70 & 45 & 20 & 45 & 40 & 40 & 40 & 45 & 30 & 35 & 30 & 30 & 35 & 40 & 40 & 45 & 30 & 40 & 30\end{array}$

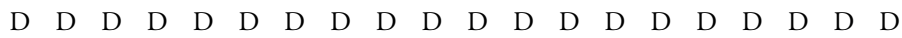
$\operatorname{Re} \operatorname{Re} \operatorname{Re} \operatorname{Re} \operatorname{Re} \operatorname{Re} \operatorname{Re} \operatorname{Re} \operatorname{Re} \operatorname{Re} \operatorname{Re} \operatorname{Re} \operatorname{Re} \operatorname{Re} \operatorname{Re} \operatorname{Re} \operatorname{Re} \operatorname{Re} \operatorname{Re} \operatorname{Re}$ $\begin{array}{llllllllllllllllllll}40 & 10 & 10 & 0 & 0 & 0 & 0 & 0 & 0 & 0 & 0 & 0 & 0 & 0 & 0 & 0 & 0 & 0 & 10 & 5\end{array}$

$\begin{array}{lllllllllllllllllllll}\mathrm{E} 3 \mathrm{~b} & 80 & 60 & 40 & 60 & 50 & 50 & 80 & 70 & 80 & 70 & 50 & 60 & 50 & 40 & 60 & 60 & 60 & 40 & 40 & 60\end{array}$

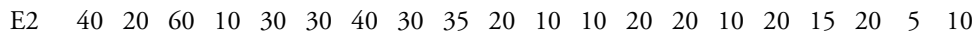
E1 $\quad 80100100100100100100 \quad 80 \quad 90 \quad 8010010095 \quad 95 \quad 10010095 \quad 90 \quad 90 \quad 95$ E0 . . 20

$\mathrm{m}^{2} \quad 400400400400400400400300200300400400400400400400400400400400$

Locality (Nahajališče)

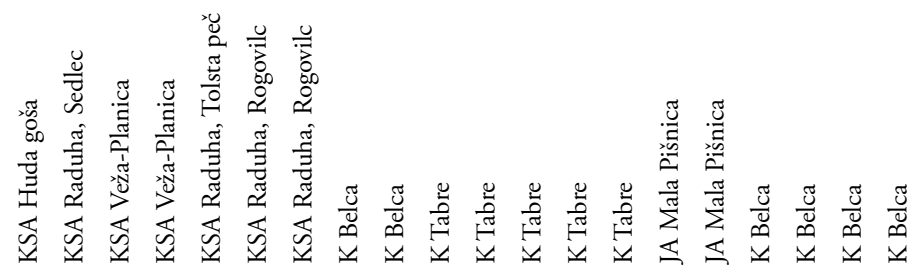

\section{Diagnostic species of the association (Diagnostične vrste asociacije)}

EP

$\mathrm{EP}$

EP

$\mathrm{EP}$

EP

$\mathrm{AF}$

ES

$\mathrm{AF}$

ES

$\mathrm{EP}$

PC

PC

TR

$\mathrm{AF}$

$\mathrm{BA}$

QP

QP

QP

QP

EP

\section{Pinus sylvestris}

Pinus sylvestris

Pinus sylvestris

Pinus sylvestris

Pinus sylvestris

Cyclamen purpurascens

Betonica alopecuros

Helleborus niger

Laserpitium peucedanoides

Rhodothamnus chamaecistus

Valeriana saxatilis

Campanula cespitosa

Hieracium porrifolium

Anemone trifolia

Salix glabra

Fraxinus ornus

Fraxinus ornus

Fraxinus ornus

Fraxinus ornus

Chamaecytisus purpureus

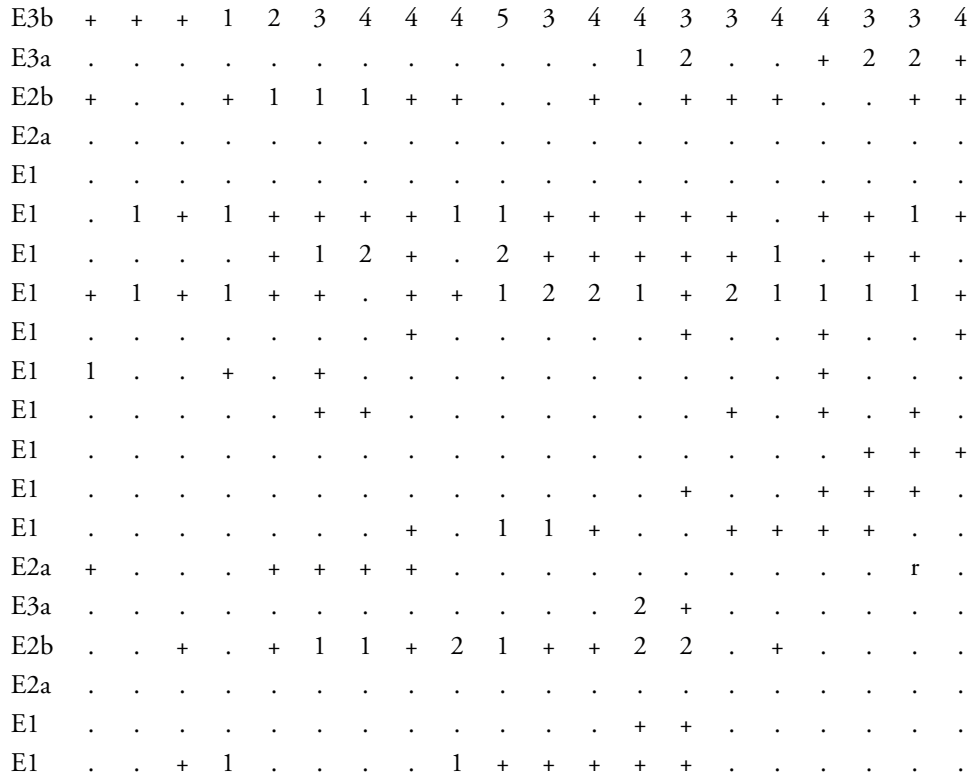




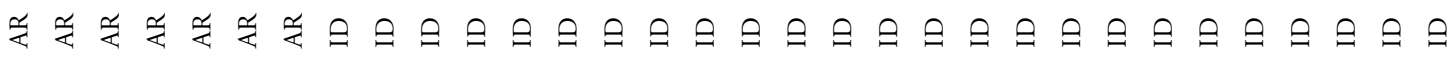

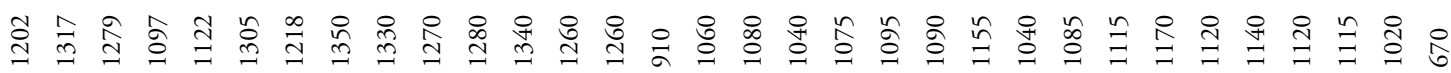

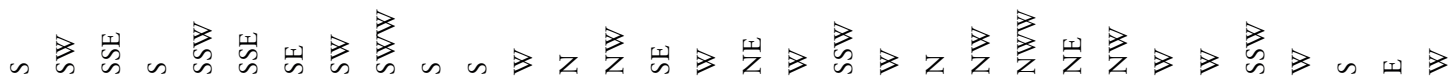

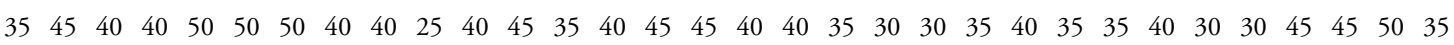

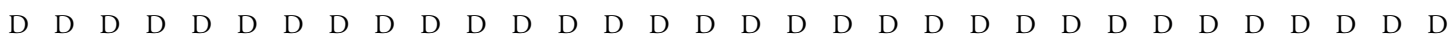
$\operatorname{Re} \operatorname{Re} \operatorname{Re} \operatorname{Re} \operatorname{Re} \operatorname{Re} \operatorname{Re} \operatorname{Re} \operatorname{Re} \operatorname{Re} \operatorname{Re} \operatorname{Re} \operatorname{Re} \operatorname{Re} \operatorname{Re} \operatorname{Re} \operatorname{Re} \operatorname{Re} \operatorname{Re} \operatorname{Re} \operatorname{Re} \operatorname{Re} \operatorname{Re} \operatorname{Re} \operatorname{Re} \operatorname{Re} \operatorname{Re} \operatorname{Re} \operatorname{Re} \operatorname{Re} \operatorname{Re} \operatorname{Re}$ $\begin{array}{llllllllllllllllllllllllllllllll}0 & 10 & 5 & 0 & 20 & 10 & 10 & 20 & 10 & 20 & 20 & 5 & 30 & 20 & 50 & 10 & 10 & 5 & 5 & 10 & 5 & 5 & 5 & 10 & 5 & 10 & 5 & 5 & 10 & 30 & 10 & 0\end{array}$

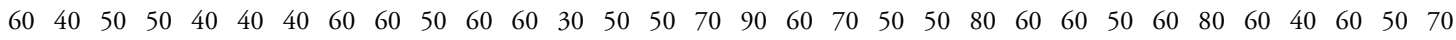
$\begin{array}{llllllllllllllllllllllllllllllll}10 & 10 & 5 & 5 & 20 & 30 & 25 & 30 & 10 & 10 & 20 & 40 & 50 & 40 & 20 & 50 & 10 & 20 & 20 & 50 & 20 & 35 & 20 & 30 & 30 & 20 & 20 & 10 & 25 & 10 & 25 & 20\end{array}$ $\begin{array}{lllllllllllllllllllllllllllllllll}95 & 90 & 95 & 95 & 80 & 90 & 90 & 70 & 80 & 80 & 80 & 80 & 70 & 80 & 70 & 80 & 90 & 95 & 100 & 100 & 100 & 95 & 100 & 100 & 95 & 90 & 100 & 100 & 90 & 60 & 95 & 90\end{array}$ $\begin{array}{lllllllllllllllllllllllll}5 & 10 & 5 & 5 & 5 & 10 & 5 & 5 & 10 & 5 & 5 & 5 & 20 & 30 & 20 & 20 & 10 & 10 & 10 & 10 & 10 & 5 & 10 & 10 & 5\end{array}$

400400400400400400400200200200200200200200200200200400400400400400400400400400400400400400400400

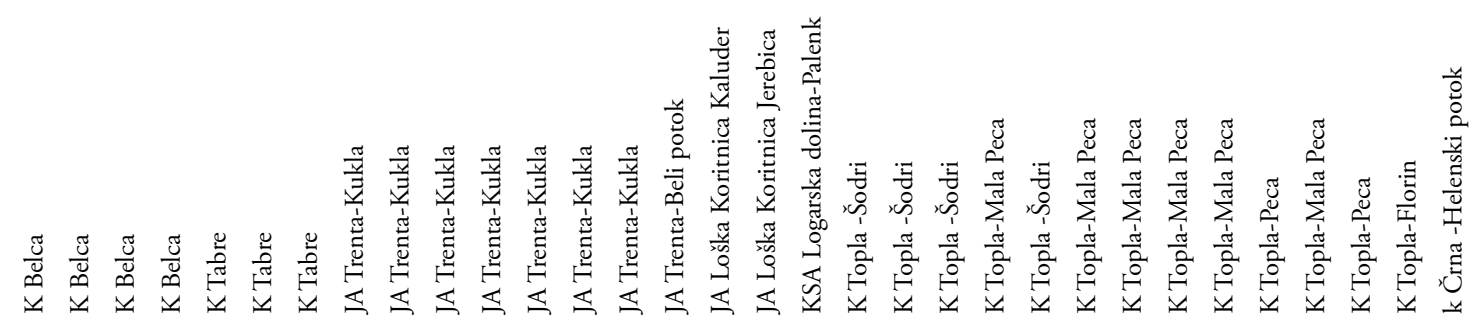

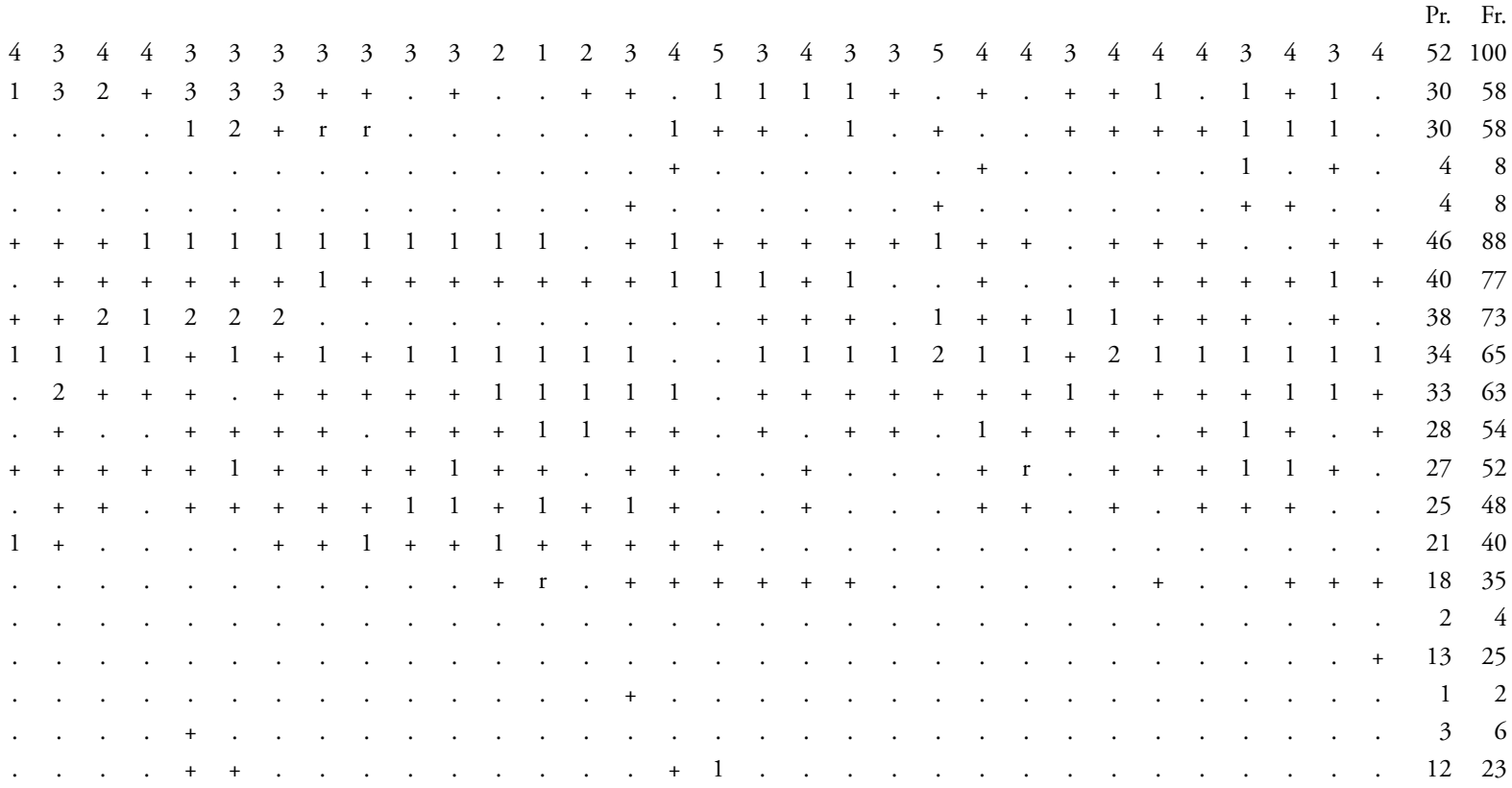




\section{$\begin{array}{llllllllllllllllllllll}\text { Number of relevé (Zaporedna štev. popisa) } & 1 & 2 & 3 & 4 & 5 & 6 & 7 & 8 & 9 & 10 & 11 & 12 & 13 & 14 & 15 & 16 & 17 & 18 & 19 & 20\end{array}$}

Differential species of lower units (Razlikovalnice nižjih enot)

$\begin{array}{ll}\text { FS } & \text { Euphorbia amygdaloides } \\ \text { FB } & \text { Euphorbia cyparissias } \\ \text { FB } & \text { Teucrium chamaedrys } \\ \text { VP } & \text { Gentiana asclepiadea } \\ \text { TG } & \text { Viola hirta } \\ \text { TG } & \text { Vincetoxicum hirundinaria } \\ \text { FB } & \text { Brachypodium rupestre } \\ \text { EP } & \text { Rhododendron hirsutum } \\ \text { VP } & \text { Vaccinium myrtillus } \\ \text { SSC } & \text { Sorbus aucuparia } \\ \text { SSC } & \text { Sorbus aucuparia } \\ \text { SSC } & \text { Sorbus aucuparia } \\ \text { SSC } & \text { Sorbus aucuparia } \\ \text { RP } & \text { Juniperus communis } \\ \text { RP } & \text { Juniperus communis } \\ \text { RP } & \text { Juniperus communis } \\ \text { EP } & \text { Erico-Pinetea }\end{array}$

Erica carnea

Calamagrostis varia

Polygala chamaebuxus

Amelanchier ovalis

Amelanchier ovalis

Amelanchier ovalis

Epipactis atrorubens

Rubus saxatilis

Cotoneaster tomentosus

Leontodon incanus

Pinus mugo

Pinus mugo

Euphrasia cuspidata

Carex alba

Gymnadenia odoratissima

Crepis slovenica

Asperula aristata

Arctostaphylos uva-ursi

Galium austriacum

Cephalanthera rubra

Allium ericetorum

Galium purpureum

Aquilegia nigricans

Genista radiata

Molinia caerulea subsp. arundinacea

Aster amellus

Carex ornithopoda

Genista januensis

Pinus nigra

Pinus nigra

Pinus nigra

Polygala nicaeensis subsp. forojulensis

Rhamnus saxatillis

Daphne cneorum

Ophrys insectifera

Euphorbia triflora subsp. kerneri?

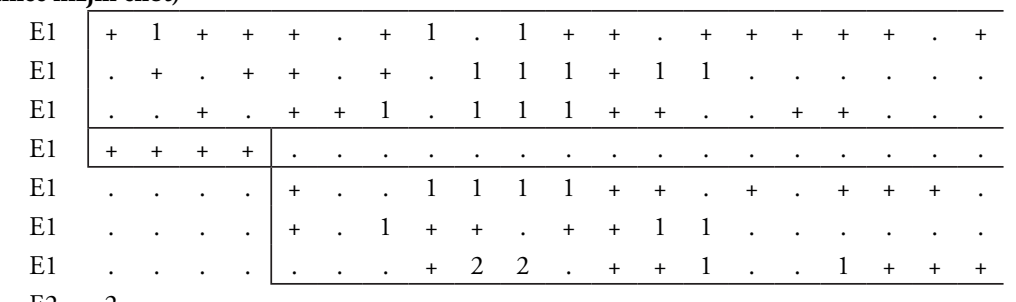

E2a 2

$\mathrm{E} 1+.11$

E3a

E2b

E2a

E1

E2b

E2a

E1

$\begin{array}{lllllllllllllllllllll}\text { E1 } & 3 & 2 & 3 & 2 & 5 & 3 & 2 & 4 & 3 & 2 & 3 & 4 & 4 & 4 & 5 & 5 & 5 & 5 & 5 & 5\end{array}$

$\begin{array}{lllllllllllllllllllll}\text { E1 } & 2 & 3 & 3 & 4 & 2 & 3 & 2 & 3 & 3 & 2 & 3 & 2 & 3 & 3 & 2 & 1 & 2 & 3 & 2 & 2\end{array}$

$\mathrm{E} 1++12+++2212121++2221$

$\mathrm{E} 2 \mathrm{~b}+.1++++22111++\ldots+$

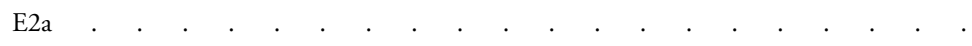

$\mathrm{E} 1$. . . . . . . . . . ..++++++

$\mathrm{E} 1+1 \cdot++++1 \cdot+++++++++++$

$\mathrm{E} 11+1+++1 .+++++\ldots+1 .+\ldots$.

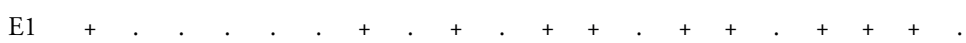

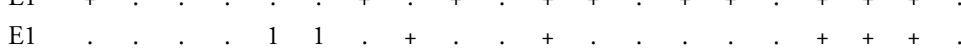

$\mathrm{E} 2 \mathrm{~b}$

E2a

E1

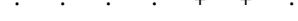

E

E

E1

E1

E1

E1

E1

E1

E1

E1

E2a

E1

E1

E1

E1

$\mathrm{E} 3 \mathrm{~b}$

E2b

E2a

E1

E1

E2b

E1

E1 


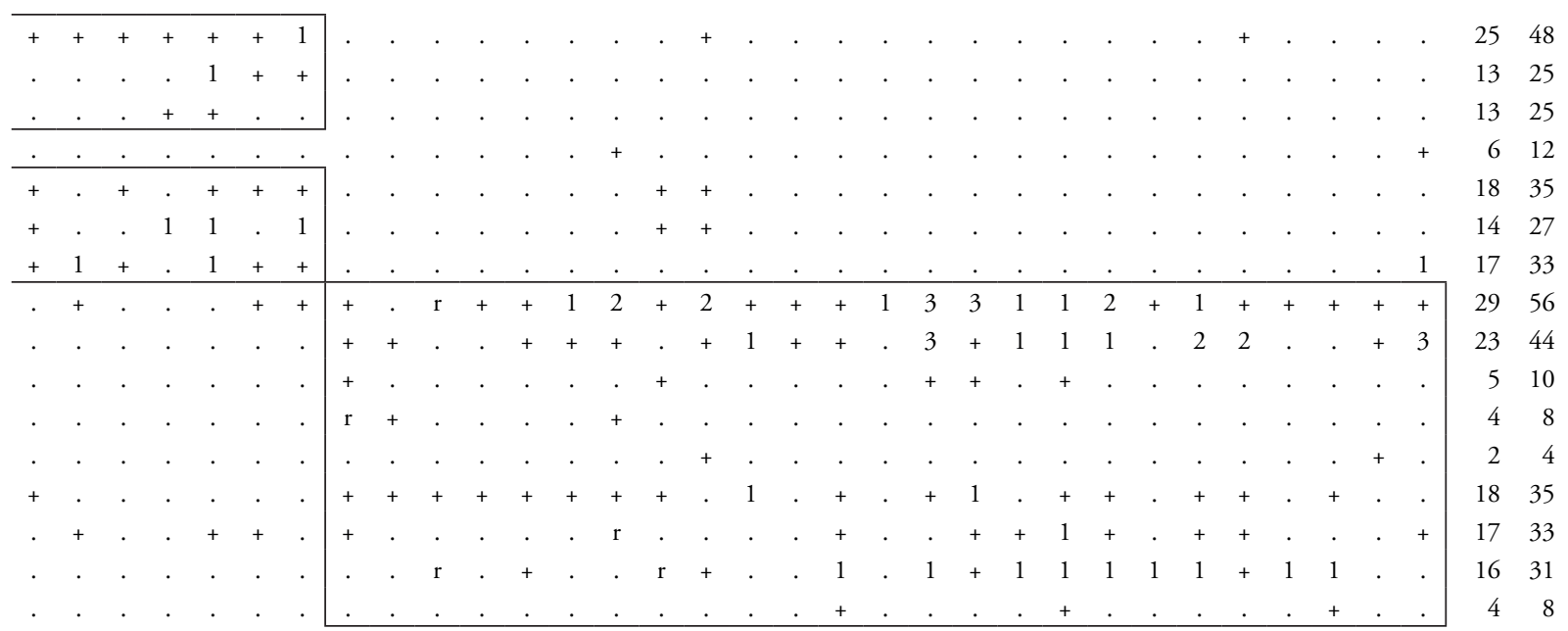

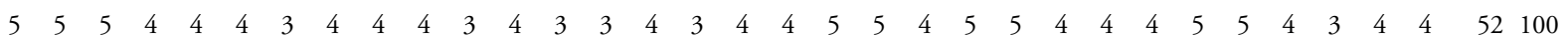

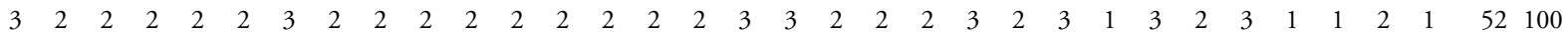

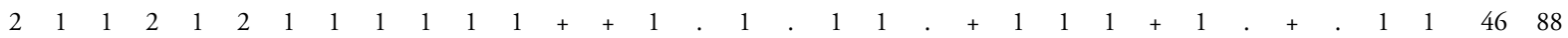

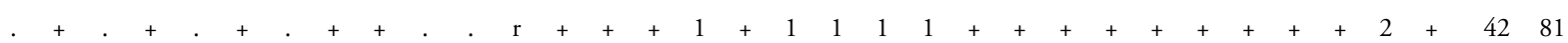

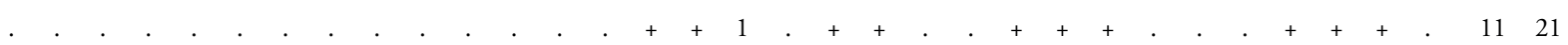

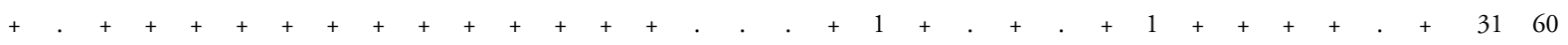

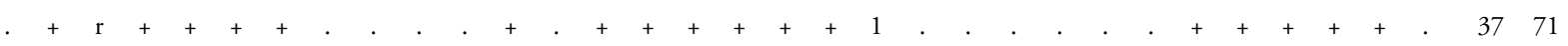

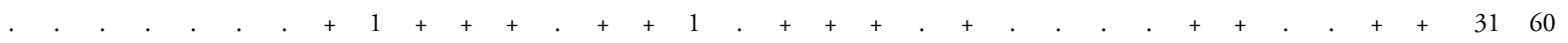

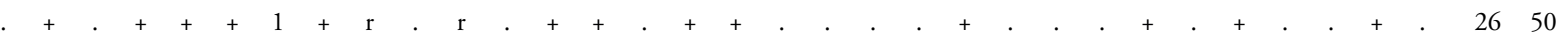

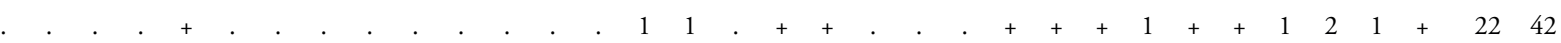

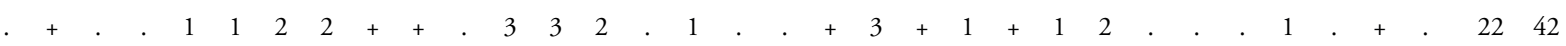

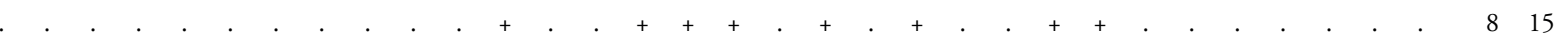

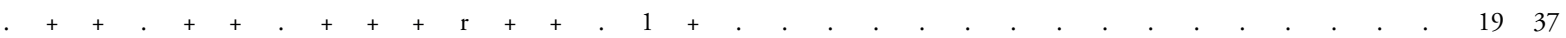

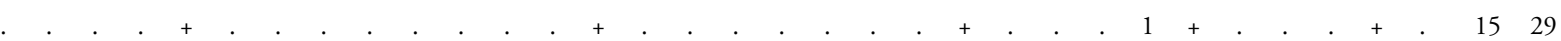

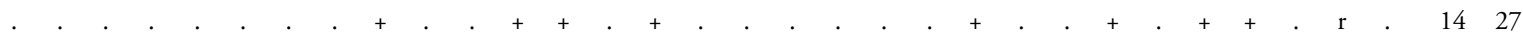

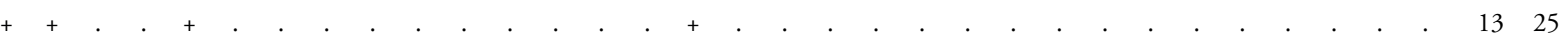

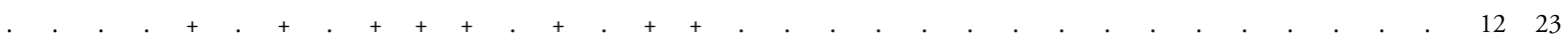

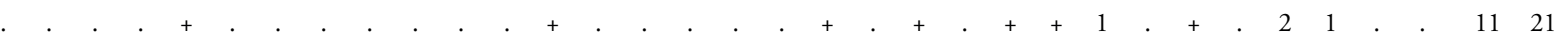

++ . . . . . . . . . . . . . . . . . . . . . . . . . . . . . . 9.17

$7 \quad 13$

713

48

48

36

2

24

24

24

24

12

12

24

24

12

12 


\section{Number of relevé (Zaporedna štev. popisa)}

VP Vaccinio-Piceetea

Picea abies

Picea abies

Picea abies

Picea abies

Picea abies

Hieracium murorum

Vaccinium vitis-idaea

Larix decidua

Larix decidua

Larix decidua

Larix decidua

Larix decidua

Valeriana tripteris

Solidago virgaurea

Aposeris foetida

Goodyera repens

Luzula sylvatica

Melampyrum sylvaticum

Pyrola minor

Clematis alpina

Homogyne sylvestris

Gymnocarpium dryopteris

Rosa pendulina

\begin{tabular}{l|cccc|}
\cline { 2 - 3 } E3b & 1 & 3 & 2 & 2 \\
E3a & $\cdot$ & $\cdot$ & $\cdot$ & $\cdot$ \\
E2b & $\cdot$ & 1 & 2 & + \\
E2a & $\cdot$ & $\cdot$ & $\cdot$ & $\cdot$ \\
E1 & $\cdot$ & + & + & $\cdot$ \\
\cline { 2 - 3 } E1 &. & + & + & 1
\end{tabular}

E1

E3b 2 \begin{tabular}{llll}
2 & 2 & 1 & 2 \\
\hline
\end{tabular}

Listera cordata

E3a

E2b

E2a

Pyrola rotundifolia

Ajuga pyramidalis

Maianthemum bifolium

Oxalis acetosella

Abies alba

Abies alba

Veronica urticifolia

Luzula luzuloides

Orthilia secunda

Pyrola media

E1

E

Homogyne alpina

Calamagrostis villosa

Huperzia selago

Diphasiastrum complanatum

Dryopteris dilatata

E1

E1

E1

E1

E1

E1

E2a

E1

E1

E2a

E1

E1

E1

E1

E1

E3b

E2b

E1

E1

E1

E1

E1

E1

E1

E1

E1

AF Aremonio-Fagion

Knautia drymeia

Rhamnus fallax

E1

E2b

FS Fagetalia sylvaticae

Acer pseudoplatanus

Acer pseudoplatanus

Acer pseudoplatanus

Acer pseudoplatanus

Daphne mezereum

Fagus sylvatica

Fagus sylvatica

Fagus sylvatica

Fagus sylvatica

Fagus sylvatica

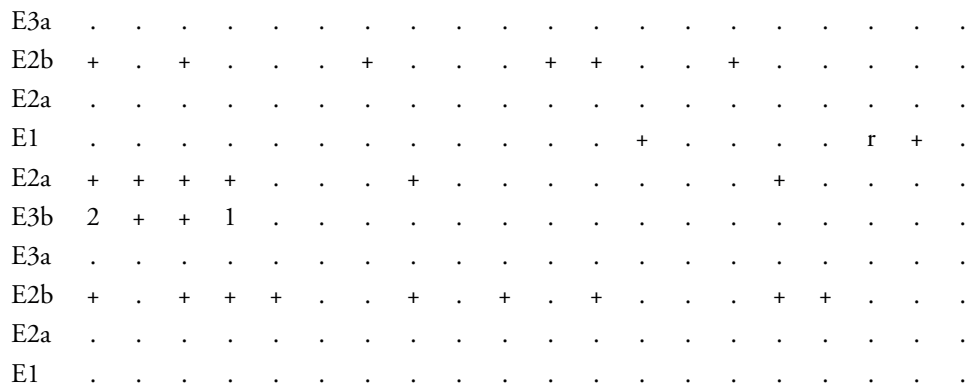




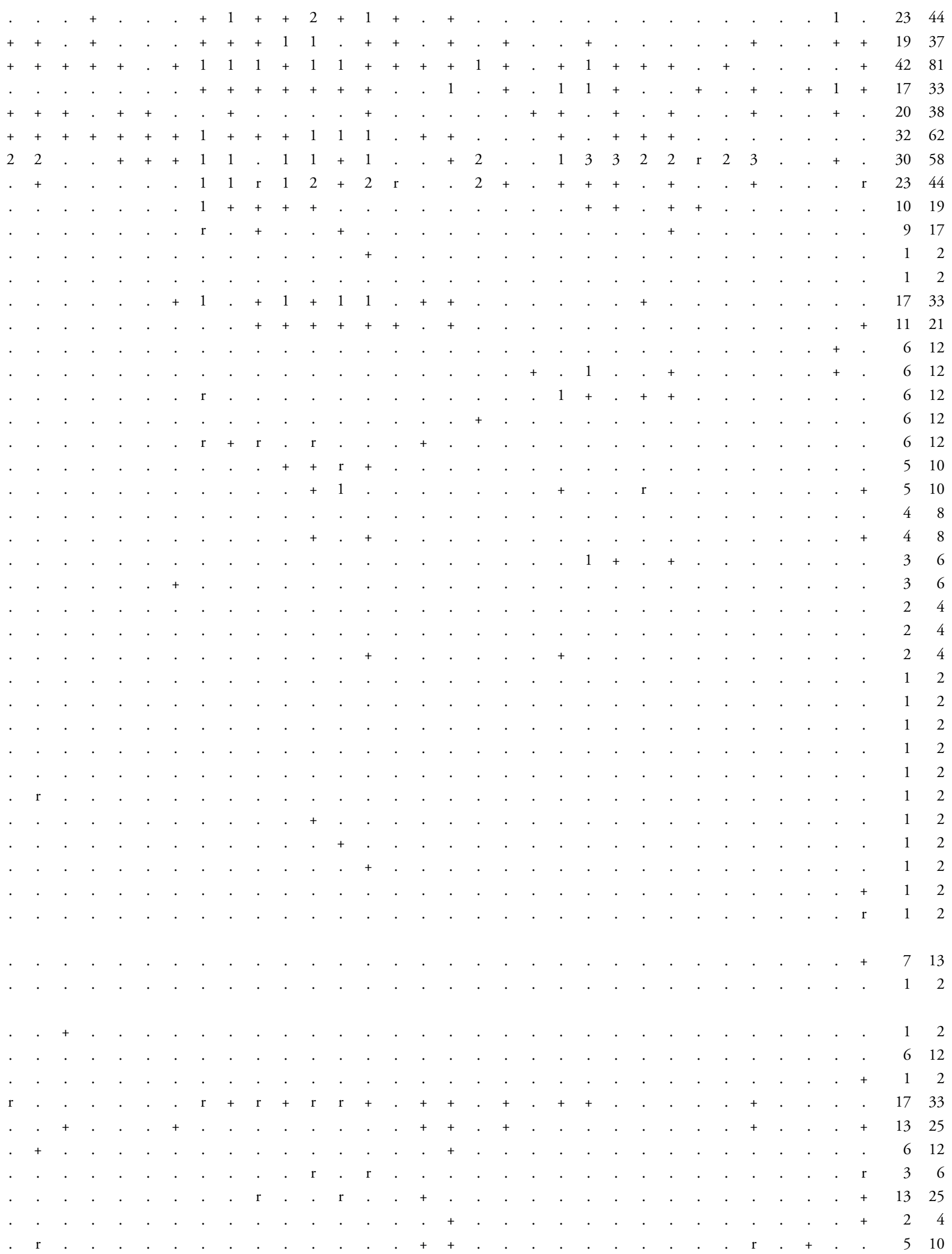




\section{Number of relevé (Zaporedna štev. popisa)}

Mercurialis perennis

Melica nutans

Neottia nidus-avis

Epipactis helleborine

Prenanthes purpurea

Laburnum alpinum

Laburnum alpinum

Laburnum alpinum

Laburnum alpinum

Luzula nivea

Viola reichenbachiana

Galium laevigatum

Lonicera alpigena

Salvia glutinosa

Cephalanthera damasonium

Campanula trachelium

Lilium martagon

Heracleum sphondylium

Mycelis muralis

Symphytum tuberosum

Brachypodium sylvaticum

Euphorbia dulcis

QP Quercetalia pubescenti-petraeae

Sorbus aria

Sorbus aria

Sorbus aria

Sorbus aria

Convallaria majalis

Ostrya carpinifolia

Ostrya carpinifolia

Ostrya carpinifolia

Ostrya carpinifolia

Hypericum montanum

Carex flacca

Lathyrus niger

Melittis melissophyllum

Epipactis muelleri

$\mathrm{QR}$ Quercetalia roboris

Pteridium aquilinum

Melampyrum pratense

Potentilla erecta

Populus tremula

QF Querco-Fagetea

Platanthera bifolia

Carex digitata

Hepatica nobilis

Cephalanthera longifolia

Corylus avellana

Cruciata glabra

Dactylorhiza fuchsii

Pyrus pyraster

Festuca heterophylla

Frangula alnus

Frangula alnus

$\begin{array}{ll} & 1 \\ \text { E1 } \\ \text { E1 } \\ \text { E1 } \\ \text { E1 } \\ \text { E1 } \\ \text { E3a } \\ \text { E2b } \\ \text { E2a } \\ \text { E1 } \\ \text { E1 } \\ \text { E1 } \\ \text { E1 } \\ \text { E2b } \\ \text { E1 } \\ \text { E1 } \\ \text { E1 } \\ \text { E1 } \\ \text { E1 } \\ \text { E1 } \\ \text { E1 } \\ \text { E1 } \\ \text { E1 }\end{array}$.

$\begin{array}{llllllllllllllllllll}1 & 2 & 3 & 4 & 5 & 6 & 7 & 8 & 9 & 10 & 11 & 12 & 13 & 14 & 15 & 16 & 17 & 18 & 19 & 20\end{array}$ 


\section{Number of relevé (Zaporedna štev. popisa)}

Listera ovata

Anemone nemorosa

Taxus baccata

SSC Sambuco-Salicion capreae

Salix caprea

Juglans regia

RP Rhamno-Prunetea

Berberis vulgaris

Viburnum lantana

Rhamnus catharticus

TG Trifolio-Geranietea

Laserpitium siler

Polygonatum odoratum

Anthericum ramosum

Digitalis grandiflora

Clinopodium vulgare

Laserpitium latifolium

Origanum vulgare

Vicia incana

Astragalus glycyphyllos

Trifolium rubens

Thalictrum minus

Iris graminea

BA Betulo-Alnetea

Salix appendiculata

Salix appendiculata

Salix appendiculata

Salix waldsteiniana

MuA Mulgedio-Aconitetea

Silene vulgaris subsp. antelopum

Phyteuma ovatum

Polygonatum verticillatum

Thalictrum aquilegiifolium

Ranunculus platanifolius

EA Epilobietea angustifolii

Fragaria vesca

Chamerion angustifolium

Potentilla recta

ES Elyno-Seslerietea

Sesleria caerulea

Thymus praecox subsp. polytrichus

Globularia cordifolia

Carex mucronata

Scabiosa lucida subsp. lucida

Phyteuma orbiculare

Senecio abrotanifolius

Aster bellidiastrum

Euphrasia salisburgensis

Dryas octopetala

Acinos alpinus

Carduus crassifolius

Campanula witasekiana

Gentiana clusii

Carduus defloratus $\begin{array}{llllllllllllllllllll}1 & 2 & 3 & 4 & 5 & 6 & 7 & 8 & 9 & 10 & 11 & 12 & 13 & 14 & 15 & 16 & 17 & 18 & 19 & 20\end{array}$

E1

E1

E3a

$\mathrm{E} 2 \mathrm{~b}$

E2a

E2b

E2b

E2b

E1

E1

E1

E1

E1

E1

E1

E1

E1

E1

E1

E1

E2b

E2a

E1

E2a

E1

E1

E1

E1

E1

E1

E1

E1

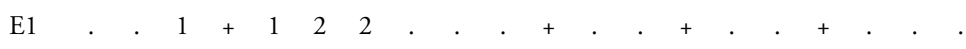

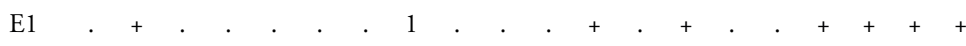

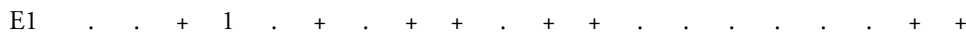

E1

E1

E1

E1

E1

E1

E1

E1

E1

E1

E1

E1 


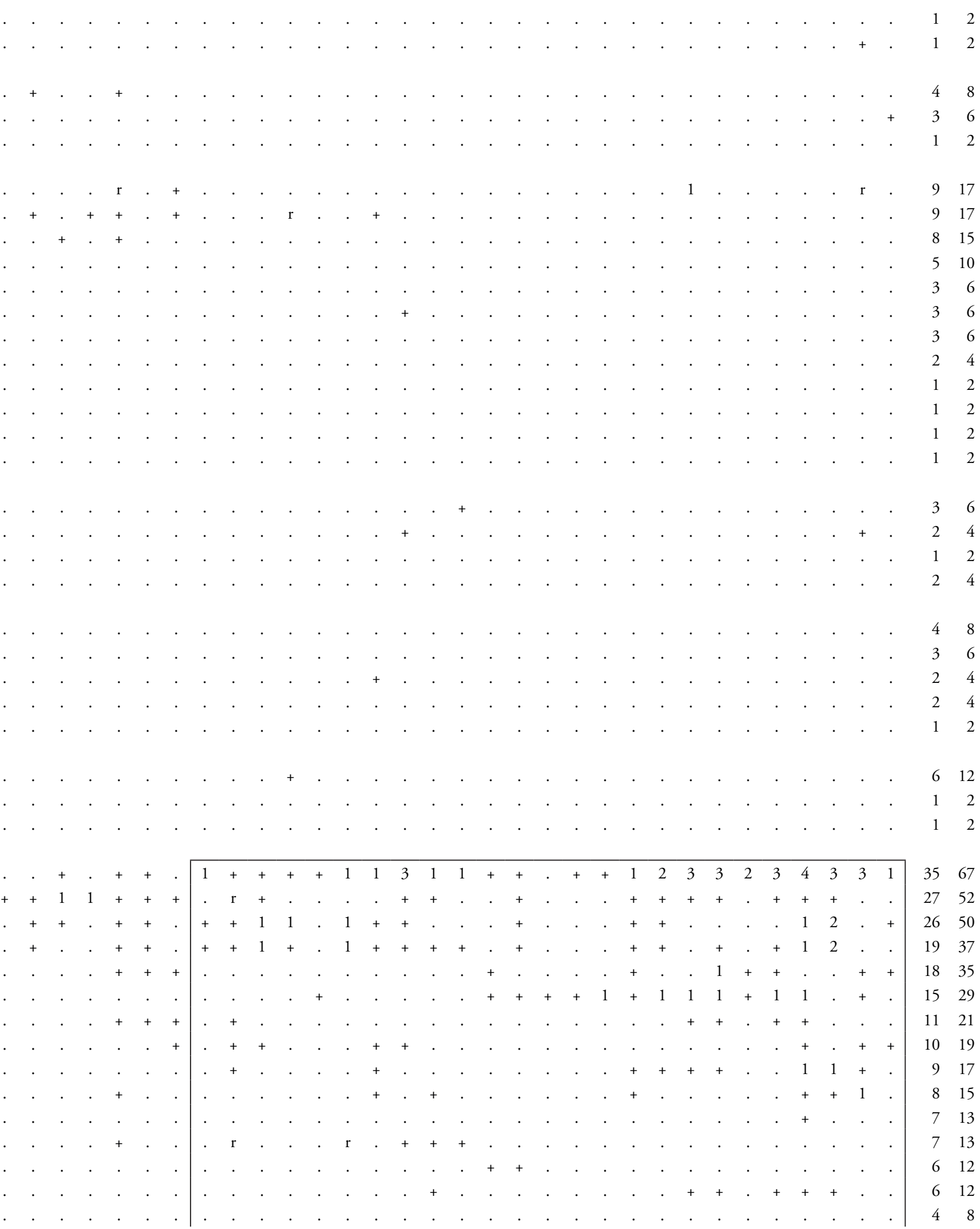




\section{Number of relevé (Zaporedna štev. popisa)}

Thesium alpinum

Carex firma

E1

Juncus monanthos

Globularia nudicaulis

Rhinanthus glacialis

Scabiosa lucida subsp. stricta

E1

Helianthemum nummularium subsp. grandiflorum E1

Polygala alpestris

Linum julicum

Helianthemum alpestre

Galium anisophyllon

E1

E1

E1

E1

Juncetea trifidi

Juncus trifidus

Campanula scheuchzeri

E1

E1

E1

E1

E1

CD Caricetalia davallianae

Tofieldia calyculata

Pinguicula alpina

E1

CU Calluno-Ulicetea

Calluna vulgaris

E1

Antennaria dioica

FB Festuco-Brometea

Buphthalmum salicifolium

Carex humilis

Carlina acaulis

Peucedanum oreoselinum

Gentianella ciliata

Linum catharticum

Cirsium erisithales

Teucrium montanum

Hippocrepis comosa

Gymnadenia conopsea

Galium lucidum

Dorycnium germanicum

Galium verum

Stachys recta

Pimpinella saxifraga

Prunella grandiflora

Cirsium pannonicum

Coronilla vaginalis

Genista germanica

$\mathrm{E} 111+1+1++1+++11++++++$

Plantago media

Cirsium acaule

Asperula cynanchica

Carlina vulgaris

Genista tinctoria

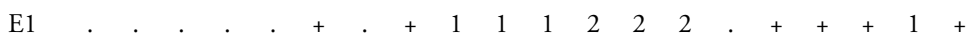

Sanguisorba minor

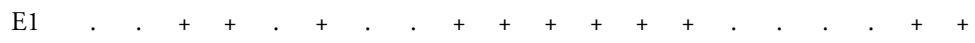

Euphrasia kerneri

Orobanche teucrii

Bromopsis erecta

Thymus praecox subsp. praecox

$$
\text { E1 }
$$

$$
\text { E1 }
$$

$$
\text { E1 }
$$$$
\text { E1 }
$$

$$
\text { E1 }
$$$$
\text { E1 }
$$

MA Molinio-Arrhenatheretea

Lotus corniculatus

Lathyrus pratensis

Galium mollugo 

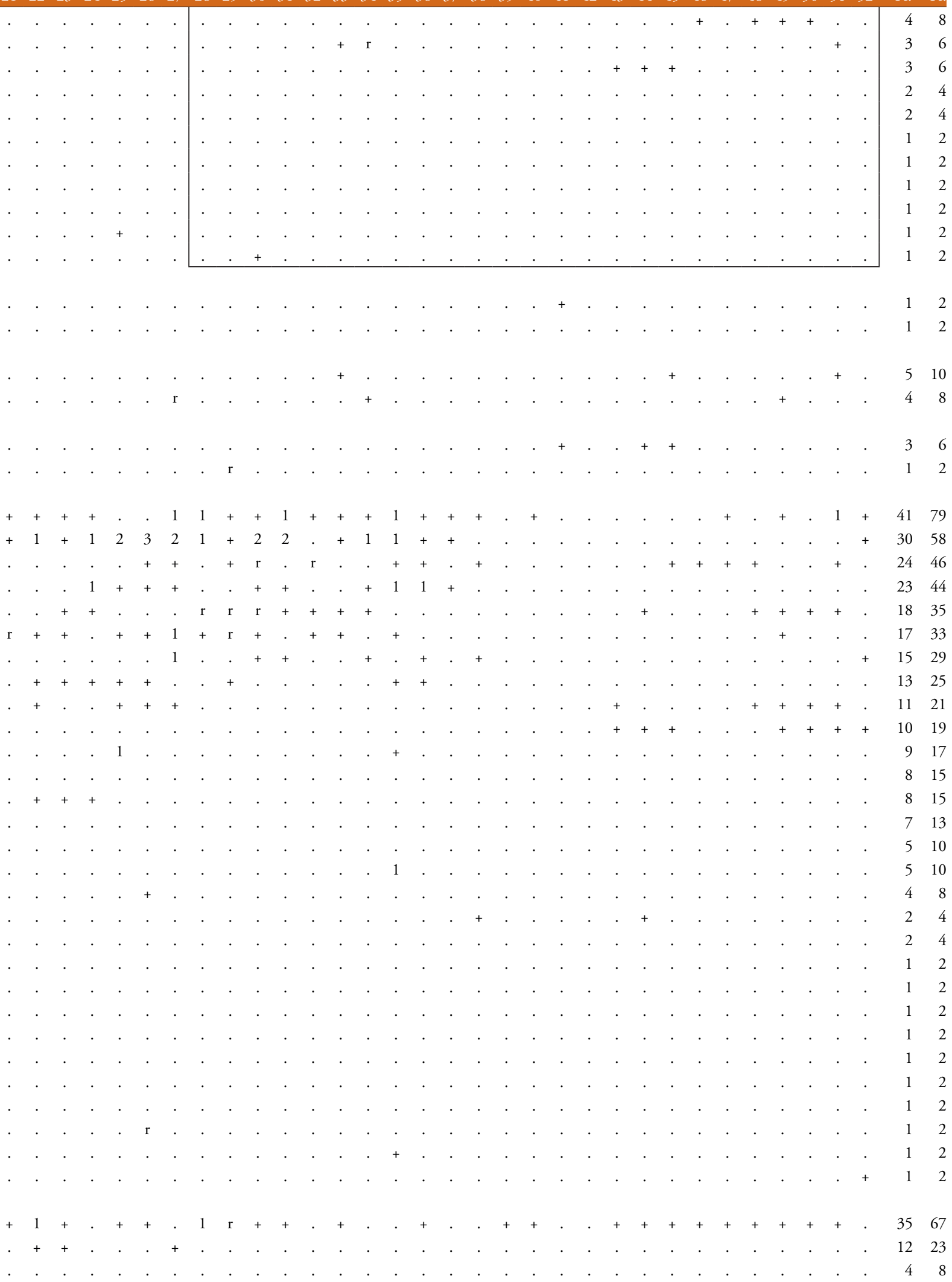


\section{Number of relevé (Zaporedna štev. popisa)}

Vicia cracca

Vicia sepium

Leucanthemum ircutianum

Plantago lanceolata

Galium album

Veronica chamaedrys

Leontodon hispidus

Succisa pratensis

TR Thlaspietea rotundifolii

Biscutella laevigata

Gymnocarpium robertianum

Hieracium bifidum

Heliosperma alpestre

Achnatherum calamagrostis

Adenostyles glabra

Hieracium glaucum

Petasites paradoxus

Rumex scutatus

Silene vulgaris subsp. glareosa

Valeriana montana

Gypsophila repens

Astrantia carniolica

Trisetum argenteum

E1
E1
E1
E1
E1
E1
E1
E1 \\ E1 \\ E \\ E1 \\ E1 \\ E1 \\ E1
}

E1

E1

E1

E1

E1

E1

E1

E1

E1

E1

E1

E1

E1

E1

PC Potentilletalia caulescentis, Physoplexido-Saxifragion

Potentilla caulescens

Primula auricula

Campanula cochleariifolia

Silene hayekiana

Rhamnus pumilus

Saxifraga squarrosa

Silene saxifraga

Paederota lutea

E1

E1

E1

E1

E1

E1

E1

AT Asplenietea trichomanis

Asplenium ruta-muraria

Dianthus sylvestris

Kernera saxatilis

Moehringia muscosa

Heliosperma pusillum

Asplenium trichomanes

Asplenium viride

E1

E1

E1

E1

E1

E1

E1

O Other species (Druge vrste)

Campanula sp.

Euphorbia sp.

Galium sp.

Carex sp.

Viola sp.

Hieracium sp.

E1

E1

E1

E1

E1

E1

ML Mosses and lichens (Mahovi in lišaji)

Tortella tortuosa

Scleropodium purum

Rhytidiadelphus triquetrus

Ctenidium molluscum

Dicranum scoparium

Hylocomium splendens

$\begin{array}{llll}\cdot & \cdot & \cdot & . \\ \cdot & \cdot & \cdot & . \\ + & \cdot & \cdot & . \\ \cdot & + & \cdot & . \\ \cdot & \cdot & \cdot & . \\ . & \cdot & \cdot & . \\ \cdot & \cdot & \cdot & . \\ . & . & . & .\end{array}$

.
.
.
.
1
.
.
.
.
.
.



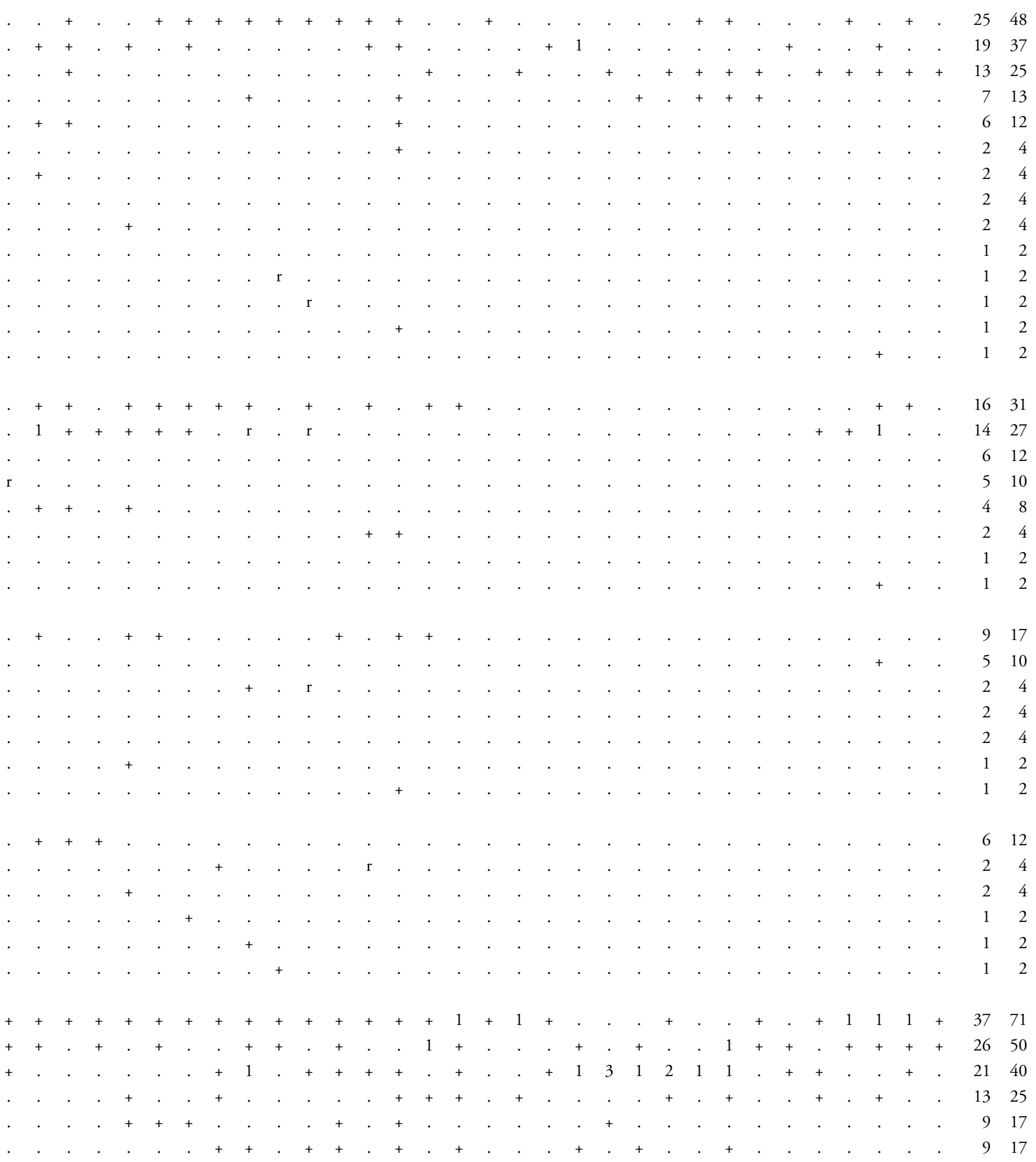


\begin{tabular}{|c|c|c|c|c|c|c|c|c|c|c|c|c|c|c|c|c|c|c|c|c|c|}
\hline Number of relevé (Zaporedna štev. popisa) & & 1 & 2 & 3 & 4 & 5 & 6 & 7 & 8 & 9 & 10 & 11 & 12 & 13 & 14 & 15 & 16 & 17 & 18 & 19 & 20 \\
\hline Neckera crispa & E0 & + & . & + & . & . & . & + & . & . & . & . & . & . & . & . & . & . & . & . & . \\
\hline Schistidium sp. & E0 & + & . & . & + & . & . & . & . & . & . & . & . & . & . & . & . & . & . & . & . \\
\hline Hypnum cupressiforme & E0 & . & + & . & + & . & . & . & . & . & . & . & . & . & . & . & . & . & . & . & . \\
\hline Pleurozium schreberi & E0 & . & . & . & . & $\cdot$ & + & . & . & . & . & . & + & . & . & . & . & + & . & . & . \\
\hline Fissidens dubius & E0 & . & . & . & . & . & . & . & . & . & . & . & . & + & . & . & . & . & . & . & . \\
\hline Isothecium alopecuroides & E0 & . & + & + & . & . & . & + & . & . & . & . & . & . & . & . & . & . & . & . & . \\
\hline Plagiochila porelloides & E0 & . & . & . & . & . & . & . & . & . & . & . & . & . & . & . & . & . & . & . & . \\
\hline Cladonia sp. & E0 & . & . & . & . & . & . & . & . & . & . & . & . & . & . & . & . & . & . & . & . \\
\hline Rhytidium rugosum & E0 & . & . & . & . & - & . & . & . & . & . & . & . & . & . & . & . & . & . & . & . \\
\hline Polytrichum formosum & E0 & . & . & + & . & . & . & . & . & . & . & . & . & . & . & . & . & . & . & . & . \\
\hline Cladonia pyxidata & E0 & . & . & . & + & - & . & . & . & . & . & . & . & . & . & . & . & . & . & . & . \\
\hline Musci spp. & E0 & . & . & . & . & . & . & . & . & . & . & . & . & + & . & . & . & . & . & . & . \\
\hline Conocephalum conicum & E0 & . & . & . & . & . & . & . & . & . & . & . & . & . & . & . & . & . & . & . & . \\
\hline Leucobryum glaucum & E0 & · & $\cdot$ & $\cdot$ & . & . & . & . & . & . & . & . & . & . & . & . & . & . & . & . & . \\
\hline Cladonia rangiferina & E0 & . & . & . & . & . & . & . & . & . & . & . & . & . & . & . & . & . & . & . & . \\
\hline Dicranella heteromalla & E0 & . & . & . & . & & . & . & . & . & . & . & . & . & . & . & . & . & . & . & . \\
\hline Hookeria lucens & E0 & . & . & . & . & - & . & . & . & . & . & . & . & . & . & . & . & . & . & . & . \\
\hline Marchantia polymorpha & E0 & . & . & . & . & . & . & . & . & . & . & . & . & . & . & . & . & . & $\cdot$ & . & . \\
\hline Solorina saccata & E0 & . & . & . & . & . & . & . & . & . & . & . & . & . & . & . & . & . & . & . & . \\
\hline
\end{tabular}

\section{Legend - Legenda}

ID Igor Dakskobler

AR Andrej Rozman

MZ Mitja Zupančič

TW Tone Wraber

VŽ Vinko Žagar

D Dolomite - dolomit

Re Rendzina - rendzina

JA Julian Alps - Julijske Alpe

KSA Kamnik-Savinja Alps - Kamniško-Savinjske Alpe

K Karavanke - Karavanke

Pr. Presence (number of relevés in which the species is presented) - število popisov, $\mathrm{v}$ katerih se pojavlja vrsta

Fr. Frequency in $\%-$ frekvenca $v \%$

? determination is questionable - določitev je vprašljiva 
. . . . . . . . . . . . . . . . . . . . . . . . . . . . . . . . . 36

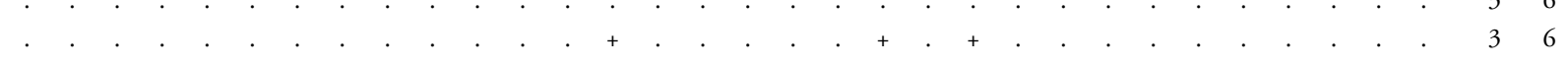

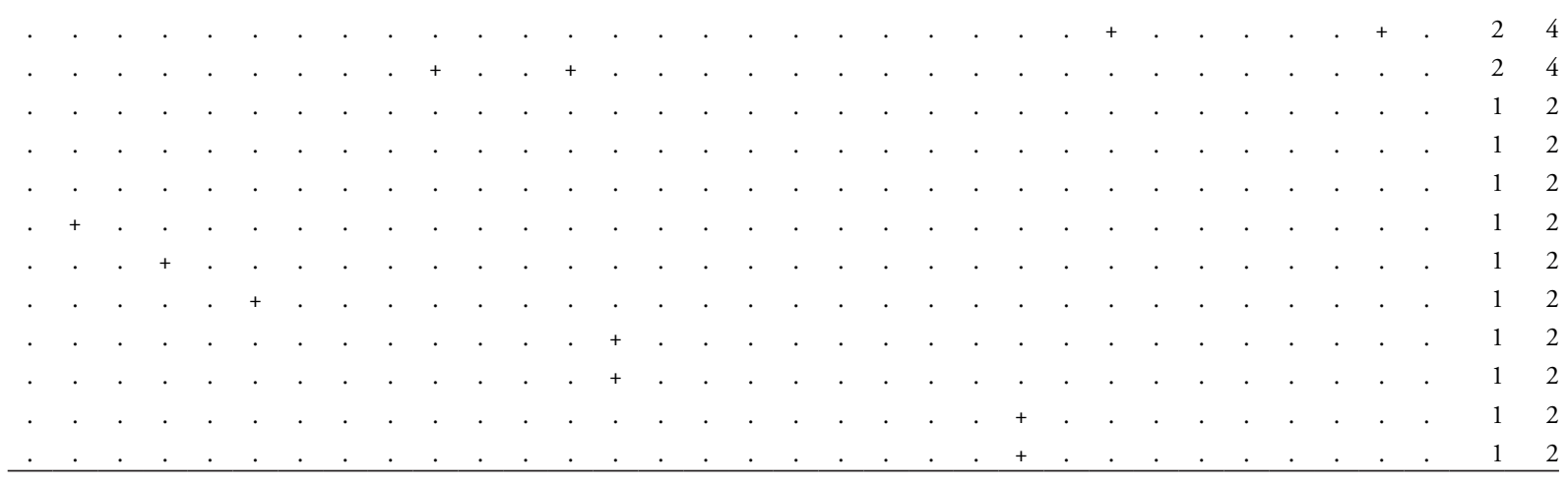


Table 2 (Tabela 2): Genisto januensis-Pinetum sylvestris campanuletosum cespitosae subass. nov. Author of the table: I. Dakskobler

\begin{tabular}{|c|c|c|c|c|c|c|c|c|c|c|c|c|c|c|c|c|c|c|c|c|c|c|c|}
\hline Vur & & 1 & 2 & 3 & 4 & 5 & 6 & 7 & 8 & 9 & 10 & 11 & 12 & 13 & 14 & 15 & 16 & 17 & 18 & 19 & 20 & 21 & 22 \\
\hline $\begin{array}{l}\text { Datab } \\
\text { (Delo }\end{array}$ & & 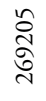 & $\begin{array}{l}\hat{n} \\
\hat{\infty} \\
\\
\hat{\sim}\end{array}$ & $\begin{array}{l}\stackrel{0}{n} \\
\hat{2} \\
\hat{\sim}\end{array}$ & $\begin{array}{l}\overline{\widehat{\lambda}} \\
\hat{\imath} \\
\hat{\imath}\end{array}$ & $\begin{array}{l}\tilde{\curvearrowright} \\
\hat{\varkappa} \\
\varkappa\end{array}$ & 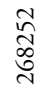 & 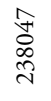 & $\begin{array}{l}\stackrel{m}{n} \\
\stackrel{\tilde{n}}{\hat{n}}\end{array}$ & 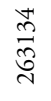 & $\begin{array}{l}\hat{n} \\
\hat{n} \\
\stackrel{\tilde{N}}{1}\end{array}$ & $\frac{\stackrel{P}{+}}{\overrightarrow{\widetilde{d}}}$ & 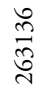 & 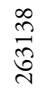 & $\begin{array}{l}\vec{\nabla} \\
\vec{n}\end{array}$ & 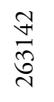 & 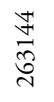 & 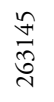 & $\overrightarrow{\widetilde{d}}$ & $\begin{array}{l}\infty \\
\stackrel{\infty}{\infty} \\
\text { ठे } \\
\text { సे }\end{array}$ & 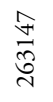 & $\begin{array}{l}\underset{\infty}{\infty} \\
\underset{\widetilde{D}}{\widetilde{N}}\end{array}$ & ్ֶర \\
\hline levat & & i̛ & in & $\stackrel{\circ}{n}$ & 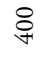 & $\stackrel{8}{\rightarrow}$ & శ్రి & & $\stackrel{\circ}{\vec{n}}$ & ్ㅗ & 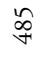 & $\overrightarrow{\widetilde{\sigma}}$ & \&్ర & Rి & 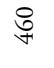 & $\stackrel{尺}{\stackrel{P}{+}}$ & $\stackrel{\circ}{\stackrel{\circ}{6}}$ & 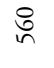 & 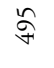 & $\stackrel{\circ}{i}$ & 옹 & 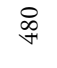 & 8 \\
\hline Aspect & & 㞱 & 岁 & $\vec{z}$ & n & $n$ & $n$ & 离 & 㭊 & 屾 & 8 & $n$ & $n$ & 齐 & 窋 & 峉 & 虫 & $n$ & 띠 & 띠 & $n$ & is & os \\
\hline Slop & & $\tilde{m}$ & 아 & $\hat{n}$ & $\stackrel{f}{f}$ & \& & 아 & $\stackrel{n}{*}$ & $\hat{n}$ & ळे & $\tilde{n}$ & లి & $\stackrel{\curvearrowleft}{\sim}$ & क् & 우 & $\mathscr{f}$ & $\mathscr{f}$ & 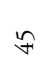 & $\tilde{n}$ & $\tilde{n}$ & $\tilde{n}$ & 아 & $m$ \\
\hline Paren & & $\mathrm{D}$ & $\mathrm{D}$ & $\mathrm{D}$ & $\mathrm{D}$ & $\mathrm{D}$ & $\mathrm{D}$ & $\mathrm{D}$ & $\mathrm{D}$ & $\mathrm{D}$ & $\mathrm{D}$ & $\mathrm{D}$ & $\mathrm{D}$ & $\mathrm{D}$ & $\mathrm{D}$ & $\mathrm{D}$ & $\mathrm{D}$ & $\mathrm{D}$ & $\mathrm{D}$ & $\mathrm{D}$ & $\mathrm{D}$ & D & D \\
\hline Soil (" & & $\operatorname{Re}$ & $\operatorname{Re}$ & $\mathrm{Re}$ & $\operatorname{Re}$ & $\operatorname{Re}$ & $\operatorname{Re}$ & $\operatorname{Re}$ & $\operatorname{Re}$ & $\operatorname{Re}$ & $\operatorname{Re}$ & $\operatorname{Re}$ & $\operatorname{Re}$ & $\operatorname{Re}$ & $\operatorname{Re}$ & $\operatorname{Re}$ & $\operatorname{Re}$ & $\operatorname{Re}$ & $\operatorname{Re}$ & $\operatorname{Re}$ & $\operatorname{Re}$ & $\mathrm{Re}$ & $\operatorname{Re}$ \\
\hline Ston & & 10 & 60 & 10 & 40 & 30 & 30 & 5 & 5 & 5 & 5 & 5 & 10 & 20 & 20 & 10 & 5 & 10 & 5 & 5 & 20 & 10 & 10 \\
\hline Cor & & & & & & & & & & & & & & & & & & & & & & & \\
\hline $\mathrm{Up}_{\mathrm{p}}$ & & 10 & 60 & 60 & 60 & 60 & 60 & 70 & 70 & 60 & 70 & 70 & 70 & 60 & 60 & 70 & 70 & 70 & 70 & 70 & 70 & 70 & 65 \\
\hline Lov & E3a & & 40 & & & & & 10 & 5 & 10 & 20 & 20 & 10 & 20 & 20 & 10 & 20 & 10 & 20 & 20 & 10 & 5 & 10 \\
\hline Sht & E2 & 50 & 30 & 30 & 50 & 40 & 30 & 10 & 20 & 30 & 20 & 20 & 30 & 30 & 20 & 20 & 20 & 20 & 20 & 20 & 20 & 20 & 20 \\
\hline & $\Gamma$ & 80 & 60 & 80 & 60 & 50 & 70 & 80 & 95 & 95 & 90 & 95 & 90 & 80 & 80 & 90 & 95 & 90 & 90 & 90 & 80 & 95 & 90 \\
\hline & $\mathrm{EC}$ & 5 & 10 & 5 & 10 & 10 & 5 & 5 & 25 & 20 & 5 & 20 & 5 & 5 & 10 & 5 & 5 & 0 & 5 & 5 & 5 & 10 & 5 \\
\hline & $\mathrm{cm}$ & 15 & 15 & 30 & 30 & 30 & 20 & 30 & 35 & 35 & 30 & 30 & 40 & 30 & 30 & 30 & 30 & 30 & 30 & 40 & 35 & 25 & 25 \\
\hline & $\mathrm{m}$ & 6 & 17 & 14 & 12 & 10 & 10 & 18 & 20 & 15 & 18 & 18 & 20 & 12 & 15 & 15 & 14 & 15 & 17 & 20 & 16 & 14 & 12 \\
\hline Num & & 22 & 67 & 77 & 49 & 67 & 47 & 52 & 38 & 55 & 61 & 52 & 46 & 50 & 45 & 48 & 44 & 44 & 32 & 37 & 32 & 38 & 30 \\
\hline $\mathrm{Re}$ & $\mathrm{m}^{2}$ & 200 & 2 & 400 & 400 & 400 & 200 & 200 & 400 & 400 & 400 & 400 & 400 & 400 & 400 & .00 & 400 & 400 & 400 & 400 & & & \\
\hline ate of tak & & $\frac{\overrightarrow{\mathrm{N}}}{\frac{N}{*}}$ & 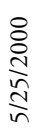 & 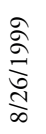 & 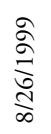 & $\frac{\stackrel{\sigma}{D}}{\stackrel{D}{\infty}}$ & $\begin{array}{l}\stackrel{\vec{a}}{\vec{N}} \\
\frac{\sqrt{*}}{a}\end{array}$ & 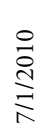 & 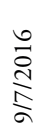 & $\begin{array}{l}\stackrel{0}{a} \\
\stackrel{ }{N} \\
\text { a }\end{array}$ & 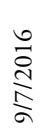 & 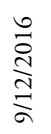 & 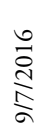 & $\frac{\stackrel{0}{\stackrel{N}{N}}}{\frac{N}{a}}$ & $\begin{array}{l}o \\
\stackrel{a}{1} \\
\stackrel{a}{a} \\
\stackrel{a}{a}\end{array}$ & $\begin{array}{l}\stackrel{0}{a} \\
\stackrel{d}{a} \\
\frac{a}{a}\end{array}$ & $\begin{array}{l}\stackrel{0}{a} \\
\stackrel{i}{a} \\
\stackrel{a}{a}\end{array}$ & $\begin{array}{l}\stackrel{0}{a} \\
\stackrel{a}{a} \\
\stackrel{a}{a}\end{array}$ & $\frac{\vec{d}}{\stackrel{a}{a}}$ & $\begin{array}{l}\stackrel{ }{a} \\
\stackrel{d}{a} \\
\text { a }\end{array}$ & $\frac{\overrightarrow{\mathrm{N}}}{\stackrel{a}{a}}$ & $\begin{array}{l}\overrightarrow{\stackrel{d}{N}} \\
\frac{a}{a}\end{array}$ & ㄱ. \\
\hline
\end{tabular}

Locality (Nahajališče)

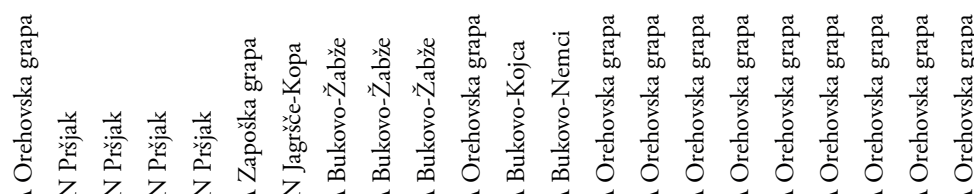

Quadrant (Kvadrant)

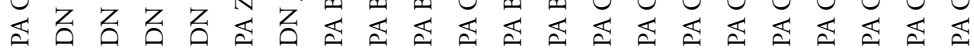

Coordinate GK Y (D-48)

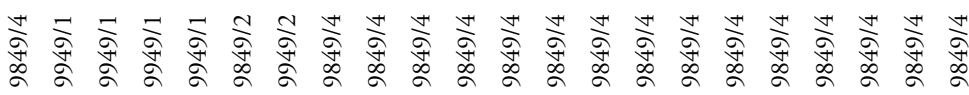

Coordinate GK X (D-48)

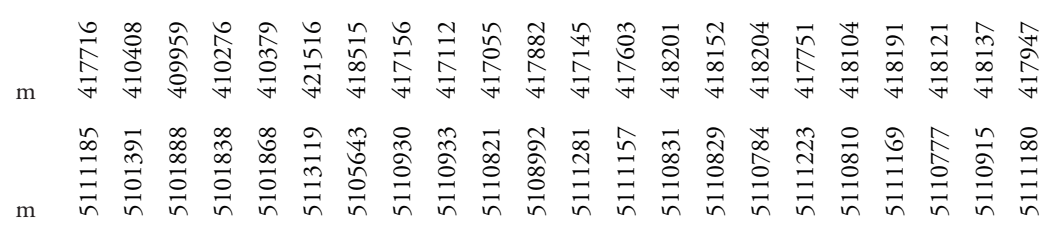

Diagnostic species of the association (Diagnostične vrste asociacije)

\begin{tabular}{|c|c|c|c|c|c|c|c|c|c|c|c|c|c|c|c|c|c|c|c|c|c|c|}
\hline EP Pinus sylvestris & E3b & + & 3 & 3 & 3 & 3 & 3 & 4 & 4 & 4 & 4 & 4 & 4 & 3 & 4 & 4 & 4 & 4 & 4 & 3 & 4 & 4 \\
\hline EP Pinus sylvestris & E3a & $\cdot$ & . & . & . & . & . & + & . & . & + & + & + & . & 1 & 1 & + & + & & . & . & 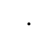 \\
\hline EP Pinus sylvestris & $\mathrm{E} 2 \mathrm{~b}$ & 4 & + & + & 1 & + & 2 & + & . & + & . & . & . & + & . & . & . & + & & + & . & . \\
\hline EP Pinus sylvestris & $\mathrm{E} 2 \mathrm{a}$ & . & . & + & 1 & 1 & . & . & . & . & . & . & . & . & . & . & . & . & & . & . & . \\
\hline EP Pinus sylvestris & E1 & . & . & . & . & . & . & . & . & . & . & . & . & . & . & . & . & . & & . & . & . \\
\hline EP Chamaecytisus purpureus & E1 & . & . & . & . & . & . & . & 1 & 1 & + & . & . & . & + & . & . & . & & + & + & + \\
\hline TR Hieracium porrifolium & E1 & . & . & . & . & + & . & . & . & + & . & . & . & . & + & . & . & + & e & . & + & . \\
\hline PC Phyteuma scheuchzeri subsp. columnae & E1 & . & 1 & . & 1 & 1 & + & . & . & . & . & . & . & + & + & + & . & . & ${ }^{\circ}$ & . & . & + \\
\hline
\end{tabular}




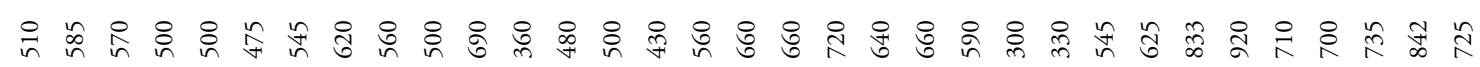

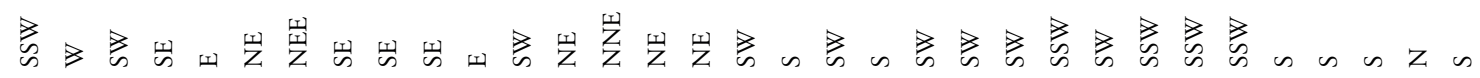

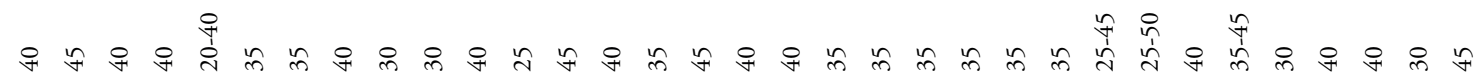

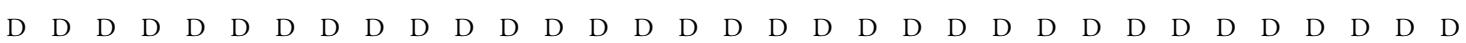
Re Re Re Re Re Re Re Re Re Re Re Re Re Re Re Re Re Re Re Re Re Re Re Re Re Re Re Re Re Re Re Re Re $\begin{array}{lllllllllllllllllllllllllllllllll}10 & 20 & 20 & 20 & 10 & 10 & 10 & 0 & 0 & 10 & 10 & 20 & 20 & 20 & 30 & 30 & 30 & 20 & 20 & 20 & 30 & 5 & 10 & 10 & 10 & 10 & 10 & 20 & 5 & 10 & 10 & 10 & 10\end{array}$

$\begin{array}{lllllllllllllllllllllllllllllllll}70 & 70 & 70 & 70 & 70 & 60 & 80 & 80 & 70 & 70 & 70 & 60 & 50 & 60 & 50 & 60 & 50 & 60 & 60 & 50 & 50 & 70 & 60 & 70 & 60 & 60 & 60 & 60 & 70 & 60 & 70 & 60 & 60\end{array}$ $\begin{array}{lllllllllllllllllllllllll}10 & 20 & 10 & 10 & 10 & 10 & 20 & 10 & 20 & 10 & 20 & 10 & 10 & 10 & 10 & 30 & 30 & 10 & 10 & 10 & 10 & 10 & 10 & 10\end{array}$

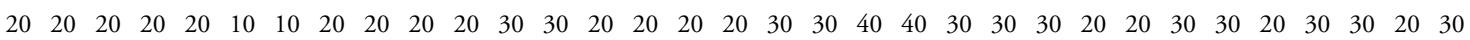
$\begin{array}{llllllllllllllllllllllllllllllllll}90 & 70 & 70 & 75 & 80 & 90 & 95 & 70 & 90 & 90 & 100 & 90 & 80 & 80 & 60 & 70 & 60 & 70 & 80 & 70 & 60 & 90 & 90 & 90 & 90 & 90 & 90 & 80 & 95 & 90 & 90 & 90 & 95\end{array}$ $\begin{array}{lllllllllllllllllllllllllllllllll}5 & 5 & 5 & 5 & 25 & 30 & 10 & 5 & 5 & 5 & 10 & 10 & 5 & 5 & 10 & 5 & 5 & 5 & 5 & 10 & 5 & 15 & 50 & 50 & 5 & 5 & 10 & 5 & 25 & 10 & 10 & 3 & 15\end{array}$ $\begin{array}{lllllllllllllllllllllllllllllllll}30 & 45 & 30 & 40 & 40 & 30 & 25 & 30 & 30 & 35 & 35 & 30 & 20 & 25 & 15 & 15 & 25 & 30 & 30 & 33 & 30 & 38 & 40 & 40 & 30 & 30 & 35 & 40 & 40 & 40 & 35 & 30 & 35\end{array}$ $\begin{array}{lllllllllllllllllllllllllllllllllll}20 & 19 & 17 & 20 & 18 & 16 & 12 & 16 & 12 & 17 & 12 & 20 & 10 & 15 & 7 & 7 & 8 & 10 & 14 & 12 & 12 & 16 & 18 & 18 & 17 & 17 & 15 & 14 & 16 & 16 & 16 & 12 & 14\end{array}$ $\begin{array}{llllllllllllllllllllllllllllllllll}32 & 40 & 38 & 38 & 45 & 33 & 51 & 49 & 51 & 55 & 46 & 56 & 40 & 40 & 42 & 30 & 56 & 48 & 51 & 55 & 57 & 62 & 68 & 73 & 47 & 38 & 40 & 45 & 32 & 36 & 45 & 26 & 40\end{array}$ 400200200200200400400200200200400200400200200200400400400400400400400400400400400400400400400200400

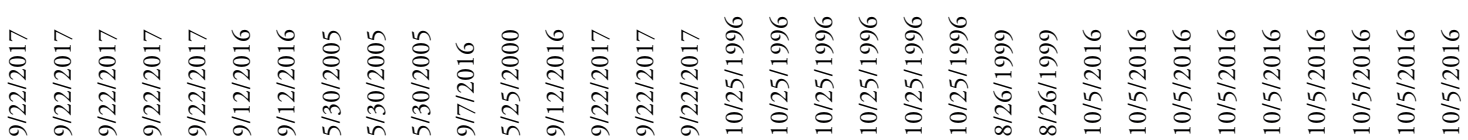

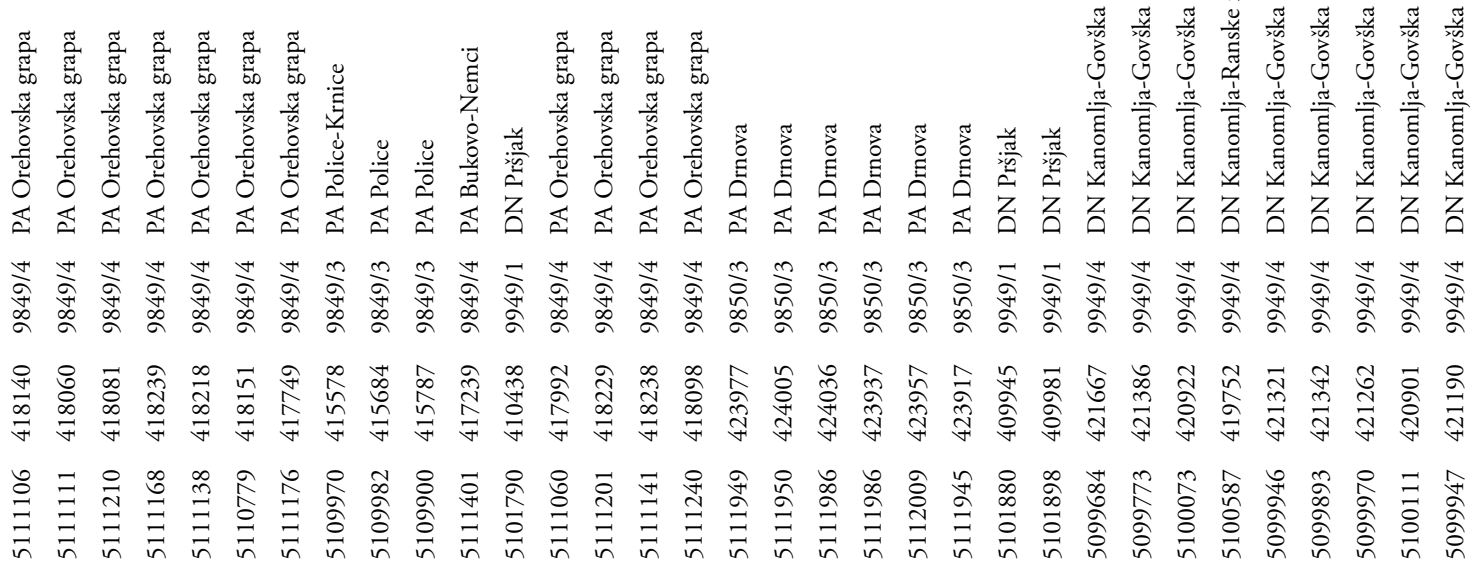

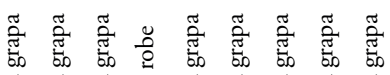

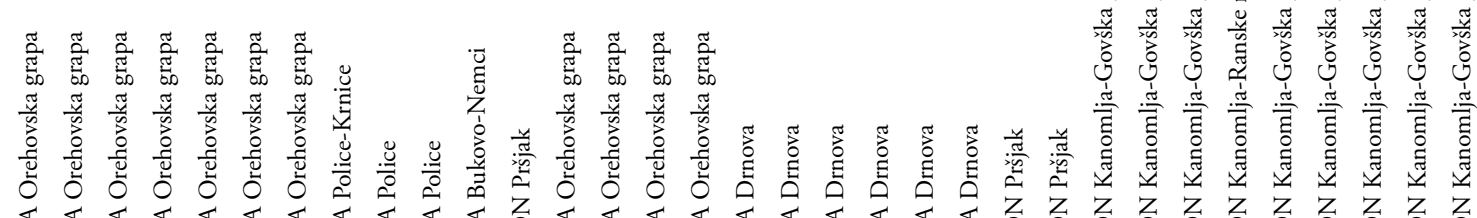

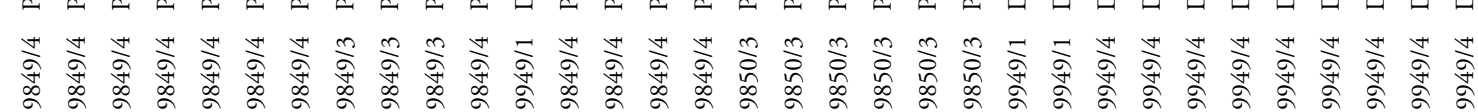

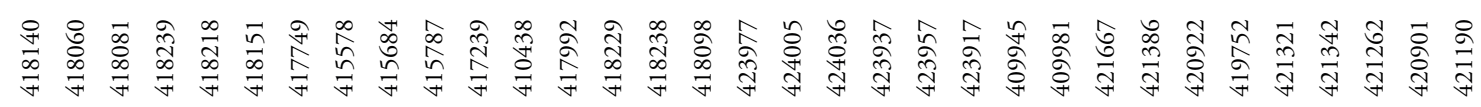

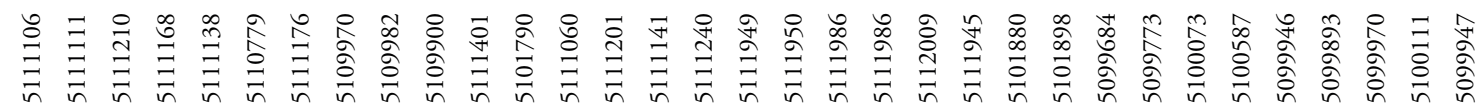

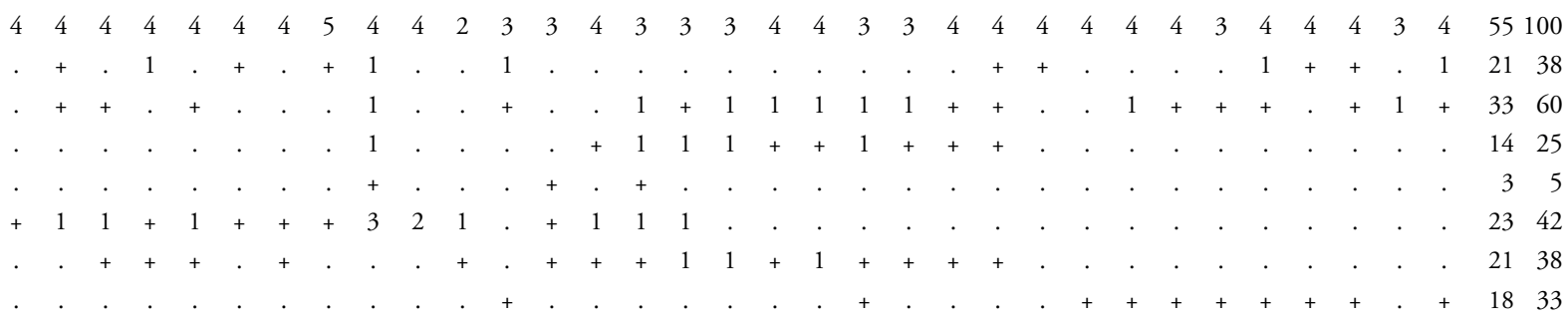




\section{Number of relevé (Zaporedna številka popisa)}

$\begin{array}{llll}\text { TG Salvia pratensis subsp. saccardiana } & \text { E1 } & \text {. } \\ \text { QP Mercurialis ovata } & \text { E1 } & \text {. } \\ \text { EP Genista januensis } & \text { E1 } & . \\ \text { FB Satureja montana subsp. variegata } & \text { E1 } & . \\ \text { FB Scabiosa hladnikiana } & \text { E1 } & . \\ \text { PC Primula carniolica } & \text { E1 } & 3\end{array}$

Differential species of the subassociation (Razlikovalnice subasociacije)

\begin{tabular}{|c|c|c|c|c|c|c|c|c|c|c|c|c|c|c|c|c|c|c|c|c|c|c|c|c|}
\hline PC & Campanula cespitosa & E1 & . & . & + & + & 1 & 1 & + & + & + & + & . & & . & + & + & + & + & + & + & + & . & + \\
\hline EP & Allium ericetorum & E1 & & . & . & . & $\cdot$ & . & + & + & + & 1 & + & + & 1 & 1 & 1 & 1 & 1 & 1 & + & 1 & 1 & 1 \\
\hline \multicolumn{25}{|c|}{ EP Erico-Pinetea } \\
\hline & Erica carnea & E1 & 4 & 3 & 4 & 3 & 3 & 3 & 4 & 5 & 4 & 4 & 5 & 1 & 4 & 4 & 4 & 4 & 3 & 4 & 4 & 4 & 4 & 4 \\
\hline & Polygala chamaebuxus & E1 & 1 & 1 & + & + & + & 1 & 1 & + & 1 & 1 & 1 & + & 1 & 1 & 1 & 1 & + & 1 & 1 & 1 & 1 & . \\
\hline & Calamagrostis varia & E1 & . & 1 & 1 & 1 & + & 1 & + & + & . & + & 1 & . & + & + & . & + & + & + & + & + & + & + \\
\hline & Leontodon incanus & E1 & . & + & + & 1 & 1 & 1 & 1 & + & + & . & . & . & . & + & + & + & + & . & . & 1 & . & + \\
\hline & Epipactis atrorubens & E1 & . & . & + & . & . & . & + & + & + & + & + & + & . & . & + & . & . & . & + & . & . & + \\
\hline & Molinia arundinacea & E1 & 3 & . & . & + & . & 1 & . & . & . & . & . & . & 2 & . & + & 2 & 3 & 2 & 1 & . & . & 1 \\
\hline & Chamaecytisus hirsutus & E1 & . & . & . & + & + & + & + & + & . & + & . & + & . & + & + & + & + & + & . & . & + & . \\
\hline & Amelanchier ovalis & $\mathrm{E} 2 \mathrm{~b}$ & . & + & . & . & . & . & 1 & . & . & . & . & . & + & . & + & + & + & . & . & . & . & . \\
\hline & Amelanchier ovalis & $\mathrm{E} 2 \mathrm{a}$ & + & . & . & . & . & . & + & . & . & . & . & . & . & . & . & . & + & . & . & . & . & . \\
\hline & Amelanchier ovalis & E1 & . & . & . & . & . & . & . & . & . & . & . & . & . & . & . & . & . & . & . & . & . & . \\
\hline & Asperula aristata & E1 & . & . & . & . & . & . & . & . & . & . & . & . & . & . & . & + & + & . & . & + & + & + \\
\hline & Carex alba & E1 & . & 1 & 1 & + & + & . & + & . & . & . & . & . & . & . & . & . & . & . & . & . & . & . \\
\hline & Galium purpureum & E1 & . & . & . & . & . & + & . & . & . & + & . & + & + & . & . & . & . & . & . & + & . & . \\
\hline & Pinus nigra & $\mathrm{E} 3 \mathrm{~b}$ & $\mathrm{r}$ & . & . & . & . & . & 1 & 1 & . & . & . & + & + & . & . & . & . & . & . & . & $\mathrm{r}$ & . \\
\hline & Pinus nigra & E3a & . & . & . & . & . & . & . & $\mathrm{r}$ & . & . & . & . & . & . & . & . & . & . & . & . & . & . \\
\hline & Pinus nigra & $\mathrm{E} 2 \mathrm{~b}$ & . & . & . & . & . & . & . & . & . & . & . & . & . & . & . & . & . & . & . & . & . & $\mathrm{r}$ \\
\hline & Pinus nigra & $\mathrm{E} 2 \mathrm{a}$ & . & . & . & . & . & . & + & . & . & . & . & . & . & . & . & . & . & . & . & . & . & . \\
\hline & Rhamnus saxatilis & $\mathrm{E} 2 \mathrm{a}$ & . & . & . & . & . & . & . & . & + & + & + & . & . & . & . & . & . & . & . & . & . & . \\
\hline & Rhamnus saxatilis & E1 & . & . & . & . & . & . & . & . & . & . & . & . & . & . & . & . & . & . & . & . & . & . \\
\hline & Aster amellus & E1 & . & . & + & . & + & . & . & . & . & . & . & . & . & . & . & . & . & . & . & . & . & . \\
\hline & Crepis slovenica & E1 & . & . & + & . & + & + & . & . & . & . & . & . & . & . & . & . & . & . & . & . & . & . \\
\hline & Genista radiata & $\mathrm{E} 2 \mathrm{a}$ & . & . & . & . & . & . & . & . & . & . & . & . & . & . & . & . & . & . & . & . & . & . \\
\hline & Peucedanum austriacum & E1 & . & . & + & . & . & . & . & . & . & . & + & . & . & . & . & . & . & . & . & . & . & . \\
\hline & Cephalanthera rubra & E1 & . & . & . & . & . & . & . & . & . & . & . & . & . & . & . & . & . & . & . & . & . & . \\
\hline & Cotoneaster tomentosus & $\mathrm{E} 2 \mathrm{~b}$ & . & . & . & . & . & . & . & . & . & . & . & . & . & . & . & . & . & . & . & . & . & . \\
\hline & Cotoneaster tomentosus & $\mathrm{E} 2 \mathrm{a}$ & . & . & . & . & . & . & . & . & . & . & . & . & . & . & . & . & . & . & . & . & . & . \\
\hline & Ophrys insectifera & E1 & . & . & . & . & . & . & . & . & . & . & . & . & . & . & . & . & . & . & . & . & . & . \\
\hline & Rhododendron hirsutum & $\mathrm{E} 2 \mathrm{a}$ & . & . & . & . & . & . & . & . & . & . & . & . & . & . & . & . & . & . & . & . & . & . \\
\hline & Rhodothamnus chamaecistus & E1 & 2 & . & . & . & . & . & . & . & . & . & . & . & . & . & . & . & . & . & . & . & . & . \\
\hline & Aquilegia nigricans & E1 & . & . & . & . & . & . & . & . & + & . & . & . & . & . & . & . & . & . & . & . & . & . \\
\hline & Rubus saxatilis & E1 & . & . & . & . & . & . & . & . & . & . & . & . & + & . & . & . & . & . & . & . & . & . \\
\hline & Pinus mugo & $\mathrm{E} 2 \mathrm{~b}$ & . & . & . & . & . & . & . & . & . & . & . & . & . & . & . & . & . & . & . & . & . & . \\
\hline & Carex ornithopoda & E1 & . & . & . & . & . & . & . & . & . & . & . & . & . & . & . & . & . & . & . & . & . & . \\
\hline & Gymnadenia odoratissima & E1 & . & . & . & . & . & . & . & . & . & . & . & . & . & . & . & . & . & . & . & . & . & . \\
\hline & Euphrasia cuspidata & E1 & . & . & . & . & . & . & . & . & . & . & . & . & . & . & . & . & . & . & . & . & . & . \\
\hline \multicolumn{25}{|c|}{ Vaccinio-Pic } \\
\hline & Picea abies & $\mathrm{E} 3 \mathrm{~b}$ & . & . & . & . & . & . & . & + & + & . & 1 & . & . & . & + & + & . & + & 1 & + & . & . \\
\hline & Picea abies & E3a & . & . & . & . & . & . & . & . & . & . & . & + & . & + & . & . & + & . & + & . & $\mathrm{r}$ & . \\
\hline & Picea abies & $\mathrm{E} 2 \mathrm{~b}$ & . & . & . & . & . & + & . & . & . & . & . & + & + & + & . & + & + & . & . & . & + & . \\
\hline & Picea abies & $\mathrm{E} 2 \mathrm{a}$ & 1 & . & . & . & . & . & + & . & . & + & + & . & . & . & + & . & . & . & . & + & . & . \\
\hline & Picea abies & E1 & . & . & . & . & . & . & . & . & . & . & . & . & . & . & . & . & . & . & . & . & . & . \\
\hline & Hieracium murorum & E1 & . & + & + & + & + & . & . & . & . & . & . & . & . & . & . & . & . & . & . & . & . & . \\
\hline & Homogyne sylvestris & E1 & . & + & . & . & . & . & . & . & . & . & . & . & . & . & . & . & . & . & . & . & . & . \\
\hline & Solidago virgaurea & E1 & . & . & . & . & . & . & . & . & . & . & . & . & . & . & . & . & . & . & . & . & . & . \\
\hline & Pyrola minor & E1 & . & . & . & . & . & . & . & . & . & . & . & . & . & . & . & . & . & . & . & . & . & . \\
\hline
\end{tabular}




\begin{tabular}{|c|c|c|c|c|c|c|c|c|c|c|c|c|c|c|c|c|c|c|c|c|c|c|c|}
\hline Number of relevé (Zaporedna številka popisa) & & 1 & 2 & 3 & 4 & 5 & 6 & 7 & 8 & 9 & 10 & 11 & 12 & 13 & 14 & 15 & 16 & 17 & 18 & 19 & 20 & 21 & 22 \\
\hline Larix decidua & E3b & . & . & . & . & . & . & . & . & . & . & . & $\mathrm{r}$ & . & . & . & . & . & . & . & . & . & . \\
\hline Larix decidua & $\mathrm{E} 2 \mathrm{a}$ & . & . & . & . & . & . & . & . & . & . & . & . & . & . & . & . & . & . & . & . & . & . \\
\hline Rosa pendulina & $\mathrm{E} 2 \mathrm{a}$ & . & . & . & . & . & . & . & . & . & . & . & . & . & . & + & . & . & . & . & . & . & . \\
\hline Valeriana tripteris & E1 & . & + & . & . & . & . & . & . & . & . & . & . & . & . & . & . & . & . & . & . & . & . \\
\hline \multicolumn{24}{|l|}{ Aremonio-Fagion } \\
\hline Cyclamen purpurascens & $\mathrm{E} 1$ & 1 & 1 & + & + & + & . & + & . & + & + & + & . & 1 & + & + & + & + & + & 1 & + & + & + \\
\hline Helleborus niger & E1 & . & 1 & + & + & + & . & + & + & + & + & + & . & + & + & 1 & 1 & 1 & + & + & + & 1 & . \\
\hline Knautia drymeia & $\mathrm{E} 1$ & . & . & + & + & . & . & . & . & . & . & . & . & . & + & . & . & + & . & . & . & . & . \\
\hline Omphalodes verna & E1 & . & + & . & . & . & . & . & . & . & . & . & . & + & . & . & . & . & . & . & . & . & . \\
\hline Anemone trifolia & $\mathrm{E} 1$ & . & 1 & . & . & . & . & + & . & . & . & . & . & . & . & . & . & . & . & . & . & . & . \\
\hline Euphorbia carniolica & $\mathrm{E} 1$ & . & + & . & . & . & . & . & . & . & . & . & . & . & . & . & . & . & . & . & . & . & . \\
\hline Primula vulgaris & E1 & . & + & + & . & . & . & . & . & . & . & . & . & . & . & . & . & . & . & . & . & . & . \\
\hline Rhamnus fallax & $\mathrm{E} 2 \mathrm{~b}$ & . & + & . & . & . & . & . & . & . & . & . & . & . & . & . & . & . & . & . & . & . & . \\
\hline Daphne laureola & $\mathrm{E} 2 \mathrm{a}$ & . & . & . & . & + & . & . & . & . & . & . & . & . & . & . & . & . & . & . & . & . & . \\
\hline Hemerocallis lilioasphodelus & E1 & . & . & . & . & . & . & . & . & . & . & . & . & . & . & . & . & . & . & . & . & . & . \\
\hline \multicolumn{24}{|l|}{ Tilio-Acerion } \\
\hline Acer pseudoplatanus & $\mathrm{E} 2 \mathrm{~b}$ & . & . & . & . & . & . & . & . & . & . & . & . & . & . & . & + & . & . & . & . & . & . \\
\hline Acer pseudoplatanus & $\mathrm{E} 2 \mathrm{a}$ & . & . & $\mathrm{r}$ & . & . & . & . & . & . & . & . & . & . & . & . & . & . & . & . & . & + & . \\
\hline Acer pseudoplatanus & E1 & . & + & . & . & . & . & . & . & + & . & . & + & . & . & + & . & . & + & + & + & + & . \\
\hline Juglans regia & $\mathrm{E} 2 \mathrm{~b}$ & . & . & . & . & . & . & . & . & . & . & . & + & . & . & . & . & . & . & . & . & . & . \\
\hline Juglans regia & $\mathrm{E} 2 \mathrm{a}$ & . & + & . & . & . & . & . & + & + & 1 & . & 1 & . & . & + & . & . & + & + & + & . & . \\
\hline Juglans regia & $\mathrm{E} 1$ & . & . & . & . & . & . & + & . & . & . & . & . & . & . & . & . & . & . & . & . & . & . \\
\hline Acer platanoides & $\mathrm{E} 2 \mathrm{a}$ & . & . & . & . & . & . & . & . & . & . & . & . & . & . & . & . & . & . & . & . & . & . \\
\hline Acer platanoides & $\mathrm{E} 1$ & . & . & . & . & . & . & . & . & . & . & . & . & . & . & . & . & . & . & . & . & . & . \\
\hline Tilia platyphyllos & $\mathrm{E} 1$ & . & . & . & . & . & . & . & . & . & . & . & + & . & . & . & . & . & . & . & . & . & . \\
\hline Euonymus latifolia & $\mathrm{E} 2 \mathrm{a}$ & . & + & . & . & . & . & . & . & . & . & . & . & . & . & . & . & . & . & . & . & . & . \\
\hline Polystichum aculeatum & E1 & . & $\mathrm{r}$ & . & . & . & . & . & . & . & . & . & . & . & . & . & . & . & . & . & . & . & . \\
\hline Aruncus dioicus & E1 & . & . & . & . & . & . & . & . & . & . & . & . & . & . & . & . & . & . & . & . & . & . \\
\hline \multicolumn{24}{|l|}{ Alnion incanae, Salicetea purpureae } \\
\hline Frangula alnus & $\mathrm{E} 2 \mathrm{~b}$ & . & . & . & . & . & . & . & . & . & . & . & . & . & . & . & . & . & . & . & . & . & . \\
\hline Frangula alnus & $\mathrm{E} 2 \mathrm{a}$ & . & + & . & + & + & . & . & + & . & + & + & . & . & . & + & . & . & . & . & . & . & . \\
\hline Frangula alnus & E1 & . & . & . & . & . & . & . & . & . & . & . & . & . & . & . & . & . & . & . & . & . & . \\
\hline Salix eleagnos & $\mathrm{E} 2 \mathrm{~b}$ & . & . & . & . & . & 1 & . & . & . & . & . & . & . & . & . & . & . & . & . & . & . & . \\
\hline Salix eleagnos & $\mathrm{E} 2 \mathrm{a}$ & . & . & . & . & . & . & . & . & . & . & . & . & . & . & . & . & . & . & . & . & . & . \\
\hline Salix purpurea & $\mathrm{E} 2 \mathrm{~b}$ & . & . & . & . & . & . & . & . & . & . & . & . & . & . & . & . & . & . & . & . & . & . \\
\hline Salix purpurea & $\mathrm{E} 2 \mathrm{a}$ & . & . & . & . & . & . & . & . & . & . & . & . & . & . & . & . & . & . & . & . & . & . \\
\hline \multicolumn{24}{|l|}{ Fagetalia sylvaticae } \\
\hline Fagus sylvatica & E3b & . & . & + & r & r & . & . & . & . & . & . & . & . & . & . & . & . & . & . & . & . & . \\
\hline Fagus sylvatica & E3a & . & + & . & . & . & . & . & . & . & . & . & . & . & . & . & . & . & . & . & . & . & . \\
\hline Fagus sylvatica & $\mathrm{E} 2 \mathrm{~b}$ & . & . & . & . & . & . & . & $r$ & . & . & + & . & . & . & . & . & . & . & . & . & . & . \\
\hline Fagus sylvatica & $\mathrm{E} 2 \mathrm{a}$ & . & . & . & . & . & . & . & . & . & $\mathrm{r}$ & . & . & . & . & . & . & . & . & . & . & . & . \\
\hline Fagus sylvatica & E1 & . & + & . & + & . & . & . & . & . & . & . & . & . & . & . & . & . & . & . & . & . & . \\
\hline Epipactis helleborine & E1 & . & . & + & . & . & . & . & + & 1 & + & . & . & . & . & . & . & . & . & . & . & . & . \\
\hline Neottia nidus-avis & $\mathrm{E} 1$ & . & . & . & . & . & . & . & . & + & + & + & . & . & . & . & . & . & . & . & . & . & . \\
\hline Salvia glutinosa & E1 & . & . & + & . & . & . & . & . & . & . & . & . & . & . & . & . & . & + & . & . & . & . \\
\hline Cephalanthera damasonium & E1 & . & . & . & . & . & . & . & . & + & + & . & . & . & . & . & . & . & . & r & . & . & . \\
\hline Laburnum alpinum & E3a & . & + & . & . & . & . & . & . & . & . & . & . & . & . & . & . & . & . & . & . & . & . \\
\hline Laburnum alpinum & $\mathrm{E} 2 \mathrm{~b}$ & . & + & . & . & . & . & . & . & . & . & . & . & . & . & . & . & . & . & . & . & . & . \\
\hline Laburnum alpinum & $\mathrm{E} 2 \mathrm{a}$ & . & . & . & . & . & . & . & . & . & . & . & . & . & . & . & . & . & . & . & . & . & . \\
\hline Daphne mezereum & $\mathrm{E} 2 \mathrm{a}$ & . & + & . & . & . & . & . & . & . & . & . & . & . & . & . & . & . & . & . & . & . & . \\
\hline Galium laevigatum & $\mathrm{E} 1$ & . & + & . & + & + & . & . & . & . & . & . & . & . & . & . & . & . & . & . & . & . & . \\
\hline Melica nutans & $\mathrm{E} 1$ & . & + & . & . & . & . & . & . & . & . & . & . & . & . & . & . & . & . & . & . & . & . \\
\hline Prunus avium & E3a & . & . & . & . & . & . & . & . & . & . & . & . & . & . & . & . & . & . & . & . & . & . \\
\hline Prunus avium & $\mathrm{E} 2 \mathrm{~b}$ & . & . & . & . & . & . & . & . & . & . & . & . & . & . & . & . & . & . & . & . & . & . \\
\hline Prunus avium & E1 & . & . & . & . & . & . & . & . & . & . & + & . & . & . & . & . & . & . & . & . & . & . \\
\hline
\end{tabular}




\begin{tabular}{|c|c|c|c|c|c|c|c|c|c|c|c|c|c|c|c|c|c|c|c|c|c|c|c|}
\hline Number of relevé (Zaporedna številka popisa) & & 1 & 2 & 3 & 4 & 5 & 6 & 7 & 8 & 9 & 10 & 11 & 12 & 13 & 14 & 15 & 16 & 17 & 18 & 19 & 20 & 21 & 22 \\
\hline Prenanthes purpurea & E1 & $\cdot$ & + & $\theta^{\circ}$ & $\cdot$ & ${ }^{\circ}$ & . & 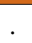 & . & $\cdot$ & . & . & . & . & . & . & . & . & . & . & . & . & . \\
\hline Viola reichenbachiana & E1 & . & . & + & . & . & . & . & . & . & . & . & . & . & . & . & . & . & . & . & . & . & . \\
\hline Lilium martagon & E1 & . & + & . & . & . & . & . & . & . & . & . & . & . & . & . & . & . & . & . & . & . & . \\
\hline Lonicera alpigena & E2a & . & + & . & . & . & . & . & . & . & . & . & . & . & . & . & . & . & . & . & . & . & . \\
\hline Mercurialis perennis & E1 & . & + & . & . & . & . & . & . & . & . & . & . & . & . & . & . & . & . & . & . & . & . \\
\hline Tilia cordata & E2a & . & . & . & . & . & . & . & . & . & . & + & . & . & . & . & . & . & . & . & . & . & . \\
\hline Mycelis muralis & E1 & . & . & . & . & . & . & . & . & . & . & . & . & . & . & . & . & . & . & . & . & . & . \\
\hline \multicolumn{24}{|l|}{ Quercetalia pubescenti-petraeae } \\
\hline Fraxinus ornus & E3b & . & . & 2 & 1 & + & 1 & . & . & . & . & . & . & . & . & . & . & + & . & . & . & . & . \\
\hline Fraxinus ornus & E3a & . & 1 & . & . & . & . & + & + & + & 1 & 1 & 1 & 1 & 1 & 1 & 1 & 1 & 1 & + & . & . & + \\
\hline Fraxinus ornus & $\mathrm{E} 2 \mathrm{~b}$ & . & 1 & 1 & 1 & 1 & 1 & + & 1 & 1 & 2 & 1 & 1 & 1 & 1 & 1 & 1 & 1 & 1 & 1 & 1 & 1 & 1 \\
\hline Fraxinus ornus & $\mathrm{E} 2 \mathrm{a}$ & . & 1 & 1 & 1 & 1 & . & 1 & . & 1 & 2 & 1 & 1 & . & 1 & 1 & 1 & 1 & . & . & 1 & 1 & + \\
\hline Fraxinus ornus & E1 & . & . & . & 1 & + & . & 1 & 1 & 1 & 1 & 1 & 1 & . & . & . & . & . & 1 & . & + & + & + \\
\hline Ostrya carpinifolia & $\mathrm{E} 3 \mathrm{~b}$ & . & 1 & 1 & 1 & + & 1 & . & . & + & + & . & + & . & . & + & + & . & + & 2 & . & . & . \\
\hline Ostrya carpinifolia & E3a & . & 1 & . & . & . & . & 1 & 1 & + & + & 1 & . & 2 & 2 & 1 & 1 & . & 1 & 1 & + & + & + \\
\hline Ostrya carpinifolia & $\mathrm{E} 2 \mathrm{~b}$ & + & . & 1 & 1 & 2 & 2 & + & . & 1 & + & . & + & 1 & . & 1 & + & + & . & 1 & 1 & 2 & 1 \\
\hline Ostrya carpinifolia & E2a & . & + & + & 1 & 1 & . & . & + & . & + & . & . & . & . & . & . & . & . & + & + & 1 & . \\
\hline Ostrya carpinifolia & E1 & . & . & . & . & . & + & . & + & . & . & . & + & . & . & . & . & . & . & . & . & . & . \\
\hline Sorbus aria & $\mathrm{E} 3 \mathrm{~b}$ & . & 1 & + & + & + & . & . & . & . & . & . & . & . & . & . & . & . & . & . & . & . & . \\
\hline Sorbus aria & E3a & . & 1 & . & . & . & . & . & . & . & + & + & + & 1 & . & . & . & . & + & + & . & . & . \\
\hline Sorbus aria & $\mathrm{E} 2 \mathrm{~b}$ & . & 1 & . & . & + & 1 & + & . & + & 1 & + & . & + & . & . & . & + & + & . & . & . & . \\
\hline Sorbus aria & E2a & . & . & . & . & . & . & + & . & . & + & . & + & . & . & . & . & . & . & . & . & . & . \\
\hline Sorbus aria & E1 & . & . & . & . & . & + & . & . & + & . & . & + & . & . & . & . & . & . & . & . & . & . \\
\hline Quercus pubescens & $\mathrm{E} 3 \mathrm{~b}$ & . & . & . & . & . & . & . & . & . & . & . & . & . & . & . & . & + & . & . & . & . & . \\
\hline Quercus pubescens & E3a & . & . & . & . & . & . & . & . & . & . & . & . & + & . & . & . & . & . & + & . & . & . \\
\hline Quercus pubescens & $\mathrm{E} 2 \mathrm{~b}$ & . & . & . & . & . & . & . & . & . & . & . & . & . & . & . & . & . & . & + & . & . & . \\
\hline Quercus pubescens & E2a & . & . & . & . & . & . & . & . & . & . & . & . & . & . & . & . & . & . & + & . & . & . \\
\hline Quercus pubescens & E1 & . & . & . & . & . & . & . & . & . & . & . & . & . & $\mathrm{r}$ & + & r & + & + & . & + & . & . \\
\hline Coronilla emerus & E2a & . & . & . & . & . & . & . & . & . & . & . & . & . & . & . & . & . & . & . & . & . & . \\
\hline Convallaria majalis & E1 & . & + & . & . & . & . & + & . & . & . & . & . & + & . & . & . & . & . & . & . & . & . \\
\hline Melittis melissophyllum & E1 & . & + & . & . & + & . & . & . & . & . & + & . & . & . & . & . & . & . & . & . & . & . \\
\hline Euonymus verrucosa & E2a & . & 1 & . & . & . & . & . & . & . & . & + & . & . & . & . & . & . & . & . & . & . & . \\
\hline Cornus mas & $\mathrm{E} 2 \mathrm{~b}$ & . & + & + & . & . & . & . & . & . & . & . & . & . & . & . & . & . & . & . & . & . & . \\
\hline Epipactis muelleri & E1 & . & . & . & . & . & . & . & . & . & . & . & . & . & . & . & . & . & . & . & . & . & . \\
\hline Sesleria autumnalis & E1 & . & . & + & . & . & . & . & . & . & . & . & . & . & . & . & . & . & . & . & . & . & . \\
\hline Carex flacca & E1 & . & . & . & . & . & . & . & . & . & . & . & . & . & . & . & . & . & . & . & . & . & . \\
\hline Cotinus coggygria & E2a & . & . & + & . & . & . & . & . & . & . & . & . & . & . & . & . & . & . & . & . & . & . \\
\hline Hypericum montanum & E1 & . & . & + & . & . & . & . & . & . & . & . & . & . & . & . & . & . & . & . & . & . & . \\
\hline Peucedanum schotti & E1 & . & . & . & . & . & 1 & . & . & . & . & . & . & . & . & . & . & . & . & . & . & . & . \\
\hline Asparagus tenuifolius & E1 & . & . & . & . & . & . & . & . & . & . & . & + & . & . & . & . & . & . & . & . & . & . \\
\hline Cornus mas & E2a & . & . & . & . & . & . & . & . & . & . & . & . & . & . & . & . & . & . & . & . & . & . \\
\hline \multicolumn{24}{|l|}{ Quercetalia roboris-petraeae } \\
\hline Quercus petraea & E1 & . & . & . & . & . & . & + & . & + & + & . & + & . & . & . & . & . & . & . & . & + & . \\
\hline Potentilla erecta & E1 & . & . & . & . & . & . & . & . & . & . & + & . & . & . & . & + & . & + & + & . & . & . \\
\hline Pteridium aquilinum & E1 & . & . & + & . & + & . & . & . & . & . & . & . & . & . & . & . & . & . & . & . & . & . \\
\hline Quercus robur & $\mathrm{E} 2 \mathrm{~b}$ & . & . & . & . & . & . & . & . & . & . & . & . & . & . & . & + & . & . & . & . & . & . \\
\hline Quercus robur & $\mathrm{E} 2 \mathrm{a}$ & . & . & . & . & . & . & . & . & . & . & . & . & . & . & . & . & . & . & . & . & . & . \\
\hline Quercus robur & E1 & . & . & . & . & . & . & . & . & . & . & + & + & . & . & . & + & . & . & . & . & . & . \\
\hline Populus tremula & $\mathrm{E} 3 \mathrm{~b}$ & . & . & . & . & . & . & . & . & . & . & . & . & . & . & . & . & . & . & . & . & . & . \\
\hline Populus tremula & E3a & . & . & . & . & . & . & . & . & . & . & . & . & . & . & . & . & . & . & . & . & . & . \\
\hline Populus tremula & $\mathrm{E} 2 \mathrm{~b}$ & . & . & . & . & . & . & . & . & . & . & . & . & . & . & . & . & . & . & . & . & . & . \\
\hline Populus tremula & $\mathrm{E} 2 \mathrm{a}$ & . & . & . & . & . & . & $\cdot$ & $\cdot$ & $\cdot$ & . & . & . & . & . & . & . & . & . & . & . & . & . \\
\hline Betula pendula & E3a & . & . & . & . & . & . & . & . & . & . & . & . & . & . & . & . & . & . & . & . & . & . \\
\hline Betula pendula & E2b & . & . & . & . & . & . & . & . & . & . & . & . & . & . & . & . & . & . & . & . & . & . \\
\hline Betonica officinalis & E1 & . & . & . & . & . & . & + & . & . & . & . & . & . & . & . & . & . & . & . & . & . & . \\
\hline
\end{tabular}




\begin{tabular}{|c|c|c|c|c|c|c|c|c|c|c|c|c|c|c|c|c|c|c|c|c|c|c|c|}
\hline Number of relevé (Zaporedna številka popisa) & & 1 & 2 & 3 & 4 & 5 & 6 & 7 & 8 & 9 & 10 & 11 & 12 & 13 & 14 & 15 & 16 & 17 & 18 & 19 & 20 & 21 & 22 \\
\hline Castanea sativa & E1 & $\cdot$ & . & $\cdot$ & $\cdot$ & $\cdot$ & $\cdot$ & $\cdot$ & $\cdot$ & . & . & . & . & . & . & . & . & . & . & . & . & . & . \\
\hline Hieracium sabaudum & E1 & . & . & + & . & . & . & . & . & . & . & . & . & . & . & . & . & . & . & . & . & . & . \\
\hline Hieracium racemosum & E1 & . & . & + & . & . & . & . & . & . & . & . & . & . & . & . & . & . & . & . & . & . & . \\
\hline Alnus glutinosa & E3a & . & . & . & . & . & . & . & . & . & . & . & . & . & . & . & . & . & . & . & . & . & . \\
\hline \multicolumn{24}{|l|}{ Querco-Fagetea } \\
\hline Hedera helix & E1 & . & + & . & . & + & . & . & . & . & + & . & . & . & . & . & . & . & . & + & + & + & . \\
\hline Corylus avellana & E1 & . & . & . & . & . & . & . & . & . & . & . & . & . & . & . & . & . & . & . & . & + & . \\
\hline Corylus avellana & E2a & . & $\mathrm{r}$ & . & . & . & . & + & + & + & + & + & + & . & . & . & . & . & . & . & . & . & . \\
\hline Listera ovata & E1 & . & + & . & . & . & . & . & . & . & . & . & . & . & . & . & . & . & . & . & . & . & . \\
\hline Carex digitata & E1 & . & + & + & + & + & . & . & . & . & . & . & . & . & . & . & . & . & . & . & . & . & . \\
\hline Clematis vitalba & $\mathrm{E} 2 \mathrm{~b}$ & . & . & + & . & . & . & . & . & . & . & . & . & . & . & . & . & . & . & . & . & . & . \\
\hline Clematis vitalba & $\mathrm{E} 2 \mathrm{a}$ & . & . & + & . & . & + & . & . & . & . & . & + & . & . & . & . & + & . & . & . & . & . \\
\hline Cephalanthera longifolia & $\mathrm{E} 1$ & . & . & . & . & . & . & . & . & + & . & + & . & . & . & . & . & . & . & r & . & . & . \\
\hline Platanthera bifolia & E1 & . & . & . & . & . & . & + & . & . & . & . & . & . & . & . & . & . & . & . & . & . & . \\
\hline Rosa arvensis & E2a & . & . & + & . & . & . & . & . & . & . & + & . & . & . & . & . & . & . & + & . & . & . \\
\hline Viscum album subsp. album & E3a & . & + & $\mathrm{r}$ & . & . & . & . & . & . & + & . & . & . & . & . & . & . & . & . & . & . & . \\
\hline Cruciata glabra & E1 & . & . & . & . & . & . & . & . & . & . & . & . & . & . & . & . & . & . & . & . & . & . \\
\hline Veratrum nigrum & E1 & . & + & . & . & . & . & . & . & . & . & . & . & + & . & . & . & . & . & . & . & . & . \\
\hline Vinca minor & E1 & . & . & + & . & . & . & . & . & . & . & . & . & . & . & . & . & . & . & . & . & . & . \\
\hline Taxus baccata & E3a & . & + & . & . & . & . & . & . & . & . & . & . & . & . & . & . & . & . & . & . & . & . \\
\hline Taxus baccata & $\mathrm{E} 2 \mathrm{~b}$ & . & + & . & . & . & . & . & . & . & . & . & . & . & . & . & . & . & . & . & . & . & . \\
\hline Hepatica nobilis & E1 & . & + & . & . & . & . & . & . & . & . & . & . & . & . & . & . & . & . & . & . & . & . \\
\hline Acer campestre & E1 & . & . & . & . & . & . & . & . & . & . & . & . & . & . & . & . & . & . & . & . & . & . \\
\hline Ilex aquifolium & $\mathrm{E} 2 \mathrm{a}$ & . & . & . & . & $\mathrm{r}$ & . & . & . & . & . & . & . & . & . & . & . & . & . & . & . & . & . \\
\hline Malus sylvestris & $\mathrm{E} 2 \mathrm{~b}$ & . & . & . & . & . & . & . & . & . & . & . & . & . & . & . & . & . & . & . & . & . & . \\
\hline Malus sylvestris & E2a & . & . & . & . & . & . & . & . & . & . & . & . & . & . & . & . & . & . & . & . & . & . \\
\hline Pyrus pyraster & E3a & . & . & . & . & . & . & . & . & . & . & . & . & . & . & . & . & . & . & . & . & . & . \\
\hline Pyrus pyraster & E2a & . & . & . & . & . & . & . & . & . & . & . & . & . & . & . & . & . & . & . & . & . & . \\
\hline Pyrus pyraster & E1 & . & . & . & . & . & . & . & . & . & . & . & . & . & . & . & . & . & . & . & . & . & . \\
\hline Lonicera xylosteum & E2a & . & . & . & . & . & . & . & . & . & . & . & . & . & . & . & . & . & . & . & . & . & . \\
\hline \multicolumn{24}{|l|}{ Sambuco-Salicion capreae } \\
\hline Salix caprea & $\mathrm{E} 2 \mathrm{~b}$ & . & . & . & . & . & . & . & . & . & . & . & . & . & . & . & . & . & . & . & . & . & . \\
\hline Sorbus aucuparia & E2a & . & + & . & . & . & . & . & . & . & . & . & . & . & . & . & . & . & . & . & . & . & . \\
\hline \multicolumn{24}{|l|}{ Rhamno-Prunetea } \\
\hline Juniperus communis & $\mathrm{E} 2 \mathrm{~b}$ & . & . & 1 & 1 & 1 & 1 & . & + & 2 & + & + & + & . & 1 & + & 1 & + & . & . & 1 & + & + \\
\hline Juniperus communis & E2a & + & + & . & 1 & . & . & + & + & 1 & + & 1 & + & . & . & + & + & . & + & 2 & 1 & 1 & 1 \\
\hline Berberis vulgaris & $\mathrm{E} 2 \mathrm{~b}$ & . & . & . & . & . & . & . & . & . & . & . & . & . & . & . & . & . & + & 1 & . & . & . \\
\hline Berberis vulgaris & E2a & . & + & . & . & + & . & . & + & + & + & + & . & + & + & . & . & . & + & 1 & + & + & . \\
\hline Berberis vulgaris & E1 & . & . & . & . & . & . & . & . & . & . & . & . & . & . & . & . & . & . & . & . & . & . \\
\hline Viburnum lantana & $\mathrm{E} 2 \mathrm{~b}$ & . & . & . & . & . & . & . & . & . & . & . & . & . & . & . & . & + & . & . & . & . & . \\
\hline Viburnum lantana & E2a & . & + & . & + & + & . & . & . & + & + & + & + & + & . & . & . & . & . & . & . & . & . \\
\hline Viburnum lantana & E1 & . & . & . & . & . & . & . & . & . & . & . & . & . & . & . & . & . & . & . & . & . & . \\
\hline Rhamnus catharticus & $\mathrm{E} 2 \mathrm{~b}$ & . & . & + & . & + & . & . & . & . & + & . & . & . & . & . & + & + & . & + & . & . & . \\
\hline Rhamnus catharticus & E2a & . & . & . & + & . & . & + & . & + & + & + & . & . & . & + & . & . & . & . & . & . & . \\
\hline Rhamnus catharticus & E1 & . & . & . & . & . & . & . & . & . & . & . & . & . & . & . & . & . & . & . & . & . & . \\
\hline Ligustrum vulgare & E1 & . & . & . & . & . & . & . & . & . & . & . & . & . & . & . & . & . & . & . & . & . & . \\
\hline Ligustrum vulgare & $\mathrm{E} 2 \mathrm{a}$ & . & . & 1 & . & + & . & . & . & . & + & + & + & . & . & . & . & . & . & . & . & . & . \\
\hline Cornus sanguinea & $\mathrm{E} 2 \mathrm{~b}$ & . & . & . & . & . & . & . & . & . & . & . & . & . & . & . & . & . & . & . & . & . & . \\
\hline Cornus sanguinea & E2a & . & . & + & . & . & . & . & . & . & . & + & . & . & . & . & . & . & . & . & . & . & . \\
\hline Rosa canina & E2a & . & . & . & . & . & . & . & . & . & . & . & + & . & . & . & . & . & . & . & . & . & . \\
\hline Crataegus monogyna & E3a & . & . & . & . & . & . & . & . & . & . & . & $\mathrm{r}$ & . & . & . & . & . & . & . & . & . & . \\
\hline Crataegus monogyna & $\mathrm{E} 2 \mathrm{~b}$ & . & . & + & . & . & . & . & . & . & . & . & . & . & . & . & . & . & . & . & . & . & . \\
\hline Crataegus monogyna & E2a & . & + & . & . & . & . & . & . & . & . & . & . & . & . & . & . & . & . & . & . & . & . \\
\hline Rubus fruticosus agg. & $\mathrm{E} 2 \mathrm{a}$ & . & . & . & . & r & . & . & . & . & . & . & . & . & . & . & . & . & + & . & . & . & . \\
\hline Euonymus europaea & E2a & . & . & + & . & . & . & . & . & . & . & . & . & . & . & . & . & . & . & . & . & . & . \\
\hline
\end{tabular}




\section{$\begin{array}{lllllllllllllllllllllllll}\text { Number of relevé (Zaporedna številka popisa) } & 1 & 2 & 3 & 4 & 5 & 6 & 7 & 8 & 9 & 10 & 11 & 12 & 13 & 14 & 15 & 16 & 17 & 18 & 19 & 20 & 21 & 22\end{array}$}

Rubus montanus

E2a

TG Trifolio-Geranietea

Vincetoxicum hirundinaria

Anthericum ramosum

Viola hirta

Thalictrum minus

Origanum vulgare

Polygonatum odoratum

Laserpitium siler

Thesium bavarum

Geranium sanguineum

Verbascum austriacum

Calamintha einseleana

Digitalis grandiflora

Grafia golaka

Clinopodium vulgare

Peucedanum cervaria

Iris graminea

Lilium carniolicum

Silene nutans

\section{(2)}

E1

E1

E1

E1

E1

E1

E1

E1

E1

E1

E1

E1

E1

E1

E1

E1

E1

E1

BA Betulo-Alnetea

Salix appendiculata

Salix appendiculata

Salix glabra

Salix glabra

EA Epilobietea angustifolii

Picris hieracioides

Atropa bella-donna

Verbascum thapsus

ES Elyno-Seslerietea

Sesleria caerulea

Globularia cordifolia

Betonica alopecuros

Carex mucronata

Euphrasia salisburgensis

Phyteuma orbiculare

Aster bellidiastrum

CD Caricetalia davallianae

Tofieldia calyculata

Pinguicula alpina

Parnassia palustris

Schoenus nigricans

FB Festuco-Brometea

Carex humilis

Buphthalmum salicifolium

Teucrium chamaedrys

Galium lucidum

Brachypodium rupestre

Euphorbia cyparissias

Teucrium montanum

Thymus praecox

Peucedanum oreoselinum

Cirsium erisithales

Galium verum

Centaurea bracteata

E1

E1

E1

E1

E1

E1

E1

E1

E1

E1

E1

E1

E1

E1

E1

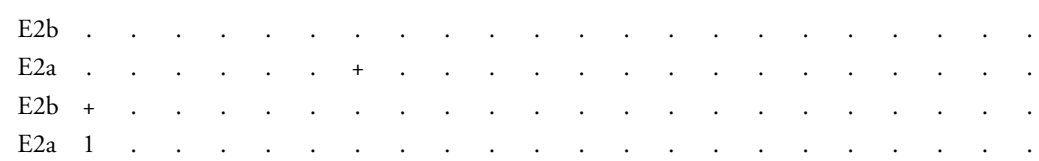

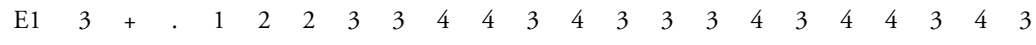

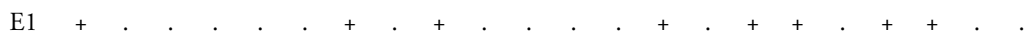

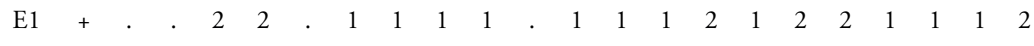

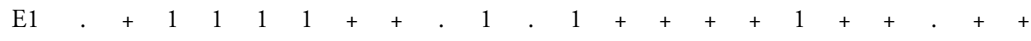

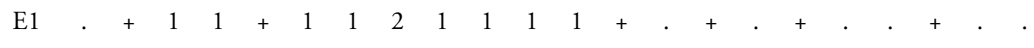

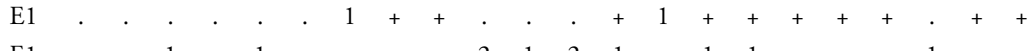

$\mathrm{E} 1 \cdot+1+1 \cdot+++2121+1+1+2$.

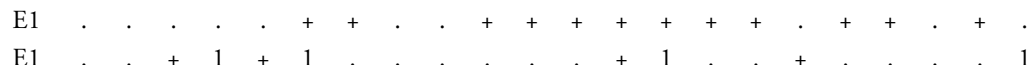

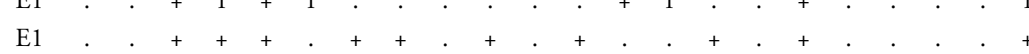

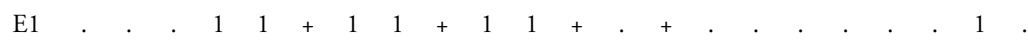




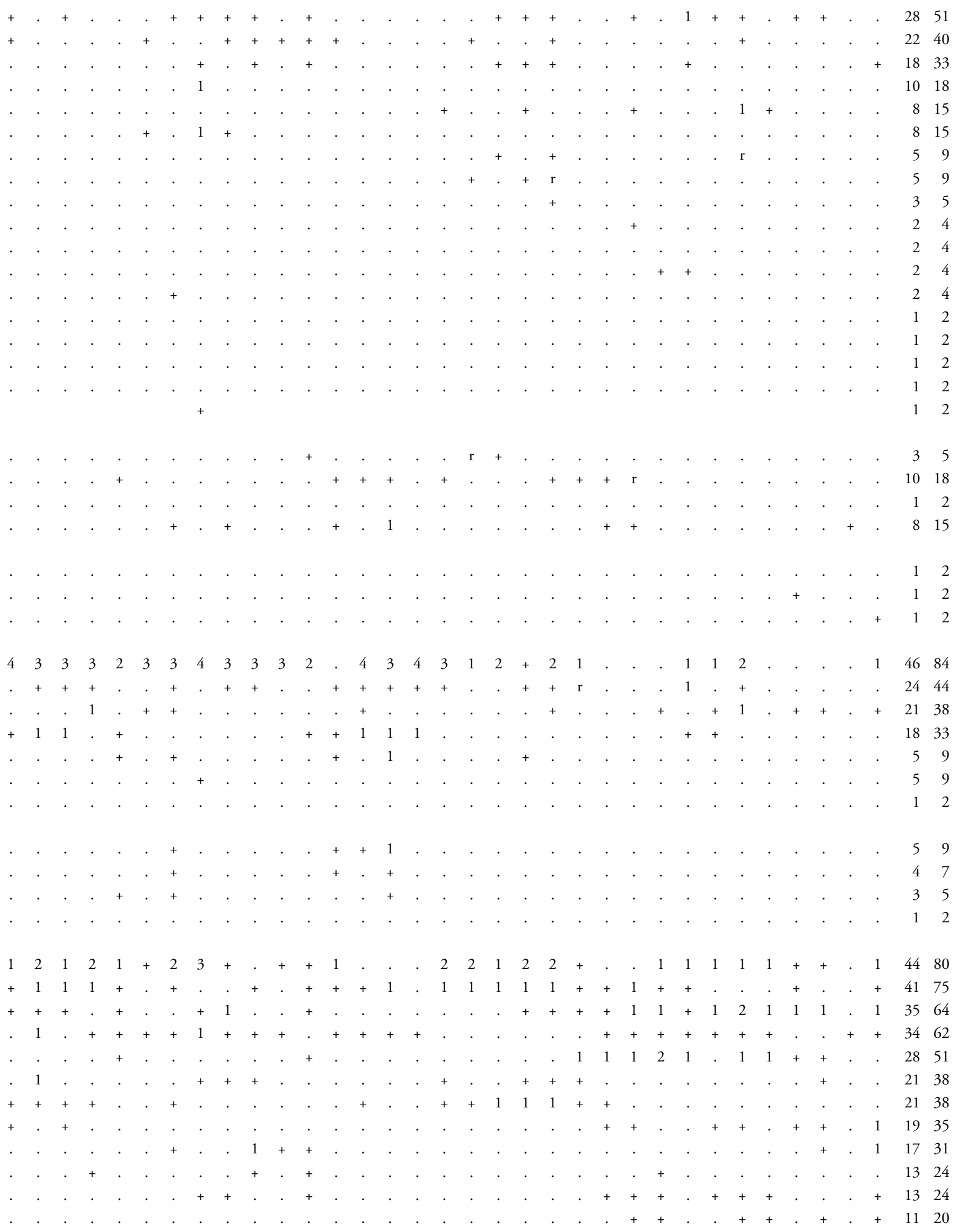




\section{$\begin{array}{llllllllllllllllllllllll}\text { Number of relevé (Zaporedna številka popisa) } & 1 & 2 & 3 & 4 & 5 & 6 & 7 & 8 & 9 & 10 & 11 & 12 & 13 & 14 & 15 & 16 & 17 & 18 & 19 & 20 & 21 & 22\end{array}$}

Carlina acaulis

Bromopsis erecta

Dianthus hyssopifolius (D. monspessulanus)

Asperula cynanchica

Cirsium pannonicum

Gentianella ciliata

Stachys recta

Thesium linophyllon

Allium carinatum subsp. pulchellum

Betonica serotina

Campanula glomerata

Hippocrepis comosa

Ranunculus polyanthemophyllus

Carlina vulgaris

Dorycnium germanicum

E1

Helianthemum nummularium subsp. obscurum E1

Koeleria pyramidata

Linum catharticum

Scabiosa triandra

Silene vulgaris subsp. vulgaris

Carduus nutans

Dorycnium herbaceum

Genista tinctoria

Hieracium pilosella

Prunella grandiflora

Veronica barrelieri

Hieracium bauhinii

Globularia punctata

Allium carinatum subsp. carinatum

Inula hirta

Centaurea fritschii

Cirsium x linkianum

Pimpinella saxifraga

Centaurium erythraea

Genista ovata

Gymnadenia conopsea

Danthonia alpina

Potentilla pusilla

MA Molinio-Arrhenetheretea

Lotus corniculatus

Dactylis glomerata

Achillea millefolium

Taraxacum officinale

TR Thlaspietea rotundifolii

Biscutella laevigata

Hieracium glaucum

Achnatherum calamagrostis

Hieracium bifidum

Petasites paradoxus

Peucedanum verticillare 


\begin{tabular}{|c|c|c|c|c|c|c|c|c|c|c|c|c|c|c|c|c|c|c|c|c|c|c|c|c|}
\hline & Number of relevé (Zaporedna številka popisa) & & 1 & 2 & 3 & 4 & 5 & 6 & 7 & 8 & 9 & 10 & 11 & 12 & 13 & 14 & 15 & 16 & 17 & 18 & 19 & 20 & 21 & 22 \\
\hline & Scrophularia juratensis & E1 & . & . & $\cdot$ & . & . & . & . & . & . & . & . & . & . & . & . & . & . & . & . & . & . & . \\
\hline & Hieracium caesium & E1 & . & . & . & . & . & . & . & . & . & . & . & . & . & . & . & . & . & . & . & . & . & . \\
\hline & Gymnocarpium robertianum & E1 & . & . & . & . & . & . & . & . & . & . & . & . & . & . & . & . & . & . & . & . & . & . \\
\hline \multirow[t]{7}{*}{$\mathrm{PC}$} & Potentilletalia caulescentis,Physoplexido-Sa & xifra & on & etr & eae & & & & & & & & & & & & & & & & & & & \\
\hline & Paederota lutea & E1 & . & 1 & . & + & + & . & . & . & . & . & . & . & . & . & . & . & . & . & . & . & . & . \\
\hline & Silene hayekiana & E1 & . & . & . & . & . & . & . & . & . & . & . & . & . & . & . & . & . & . & . & . & . & . \\
\hline & Valeriana saxatilis & E1 & . & . & . & . & . & . & . & . & . & . & . & . & . & . & . & . & . & . & . & . & . & . \\
\hline & Potentilla caulescens & E1 & . & . & . & . & . & . & . & . & . & . & . & . & + & . & . & . & . & . & . & . & . & . \\
\hline & Athamanta turbith & E1 & . & . & . & + & . & . & . & . & . & . & . & . & . & . & . & . & . & . & . & . & . & . \\
\hline & Hieracium pospichalii & E1 & . & . & . & . & . & . & . & . & . & . & + & . & . & . & . & . & . & . & . & . & . & . \\
\hline \multirow[t]{8}{*}{ AT } & Asplenietea trichomanis & & & & & & & & & & & & & & & & & & & & & & & \\
\hline & Asplenium ruta-muraria & E1 & . & + & + & + & + & . & . & + & + & + & . & + & + & . & + & . & . & . & . & . & . & . \\
\hline & Sedum album & E1 & . & . & . & . & . & . & . & . & . & . & . & . & + & . & . & . & . & . & . & . & . & . \\
\hline & Asplenium trichomanes & E1 & . & . & . & . & . & . & . & . & . & . & . & . & . & . & . & . & . & . & . & . & . & . \\
\hline & Carex brachystachys & E1 & . & + & . & . & . & . & . & . & . & . & . & . & . & . & . & . & . & . & . & . & . & . \\
\hline & Kernera saxatilis & E1 & . & . & . & . & . & . & . & . & + & . & . & . & . & . & . & . & . & . & . & . & . & . \\
\hline & Erysimum sylvestre & E1 & . & . & . & . & . & . & . & . & . & . & . & . & . & . & . & . & . & . & . & . & . & . \\
\hline & Polypodium vulgare & E1 & . & . & . & . & . & . & . & . & . & . & . & . & . & . & . & . & . & . & . & . & . & . \\
\hline \multirow[t]{3}{*}{$\mathrm{O}$} & Other species (Druge vrste) & & & & & & & & & & & & & & & & & & & & & & & \\
\hline & Festuca sp. & E1 & . & . & . & . & . & . & . & . & . & . & . & . & . & . & . & . & . & . & . & . & . & . \\
\hline & Hieracium sp. & E1 & . & . & . & . & . & . & . & . & . & . & . & . & . & . & . & . & . & . & . & . & . & . \\
\hline \multicolumn{25}{|c|}{ ML Mosses and lichens (Mahovi in lišaji) } \\
\hline & Scleropodium purum & E0 & 1 & . & + & + & + & . & 1 & + & 2 & + & . & . & 1 & . & + & + & . & + & 1 & + & 2 & + \\
\hline & Tortella tortuosa & E0 & . & + & + & + & + & + & . & + & + & . & . & . & + & . & + & . & . & . & + & + & . & . \\
\hline & Ctenidium molluscum & E0 & . & + & . & . & + & . & . & . & . & + & . & . & + & . & . & . & . & . & . & . & . & . \\
\hline & Neckera crispa & E0 & + & + & + & + & + & . & . & . & . & . & . & . & + & + & + & + & . & . & + & . & + & . \\
\hline & Hypnum cupressiforme & E0 & . & . & + & . & . & . & . & . & + & . & + & . & . & + & . & . & . & . & . & . & . & . \\
\hline & Rhytidiadelphus triquetrus & E0 & . & . & . & . & . & . & . & . & + & . & . & . & + & . & . & + & . & . & . & . & . & . \\
\hline & Hylocomium splendens & E0 & . & . & . & . & . & . & . & . & . & . & . & . & . & . & . & + & . & . & . & . & . & . \\
\hline & Fissidens dubius & E0 & . & . & . & . & . & . & . & . & . & . & . & . & . & . & . & . & . & . & . & . & . & . \\
\hline & Homalothecium lutescens & E0 & . & . & . & . & . & . & . & . & . & + & . & . & + & + & . & . & . & . & . & . & . & . \\
\hline & Schistidium apocarpum & E0 & . & . & + & . & + & . & . & . & . & . & . & . & . & . & . & . & . & . & . & . & . & . \\
\hline & Thuidium tamariscinum & E0 & . & . & + & . & . & . & . & . & . & . & . & . & . & . & . & . & . & . & . & . & . & . \\
\hline & Dicranum scoparium & E0 & . & + & . & . & . & . & . & . & . & . & . & . & . & . & . & . & . & . & . & . & . & . \\
\hline & Homalothecium sericeum & E0 & . & . & . & + & + & . & . & . & . & . & . & . & . & . & . & . & . & . & . & . & . & . \\
\hline & Pleurozium schreberi & E0 & . & . & . & . & . & . & . & . & . & . & . & . & . & . & . & . & . & . & . & . & . & . \\
\hline & Rhytidium rugosum & E0 & . & . & . & . & . & . & . & . & . & . & . & . & . & . & . & . & . & . & . & . & . & . \\
\hline & Thuidium abietinum & E0 & . & . & . & . & . & . & . & . & . & . & . & . & . & . & . & . & . & . & . & . & . & . \\
\hline & Plagiochila porelloides & E0 & . & + & . & . & . & . & . & . & . & . & . & . & . & . & . & . & . & . & . & . & . & . \\
\hline & Isothecium alopecuroides & E0 & . & . & + & . & . & . & . & . & . & . & . & . & . & . & . & . & . & . & . & . & . & . \\
\hline & Neckera complanata & E0 & . & . & . & . & . & . & . & . & . & . & . & + & . & . & . & . & . & . & . & . & . & . \\
\hline & Loeskebryum brevirostre & E0 & . & . & . & . & . & . & . & . & . & . & . & . & . & . & . & + & . & . & . & . & . & . \\
\hline & Leucobryum glaucum & E0 & . & . & . & . & . & . & . & . & . & . & . & . & . & . & . & . & . & . & . & . & . & . \\
\hline & Hypnum jutlandicum & E0 & . & . & . & . & . & . & . & . & . & . & . & . & . & . & . & . & . & . & . & . & . & . \\
\hline & Campylium stellatum & E0 & . & . & . & . & . & . & . & . & . & . & . & . & . & . & . & . & . & . & . & . & . & . \\
\hline & Encalypta streptocarpa & E0 & . & . & . & . & . & . & . & . & . & . & . & . & . & . & . & . & . & . & . & . & . & . \\
\hline & Thuidium delicatulum & E0 & . & . & . & . & . & . & . & . & . & . & . & . & . & . & . & . & . & . & . & . & . & . \\
\hline & Eurhynchium striatum & E0 & . & . & . & . & . & . & . & . & . & . & . & . & . & . & . & . & . & . & . & . & . & . \\
\hline
\end{tabular}

\section{Legend - Legenda}

D Dolomite - dolomit

Re Rendzina - rendzina

PA Pre-Alpine phytogeographical region - predalpsko fitogeografsko območje
DN Dinaric phytogeographical region - dinarsko fitogeografsko območje

Pr. Presence (number of relevés in which the species is presented) - število popisov, v katerih se pojavlja vrsta

Fr. Frequency in $\%$ - frekvenca $v \%$ 
Table 3: Synoptyc table of syntaxa Rhodothamno chamaecisti-Pinetum sylvestris, Fraxino orni-Pinetum nigrae pinetosum sylvestris, Genisto januensis-Pinetum sylvestris and Erico-Pinetum sylvestris.

Tabela 3: Sintezna tabela sintaksonov Rhodothamno chamaecisti-Pinetum sylvestris, Fraxino orni-Pinetum nigrae pinetosum sylvestris, Genisto januensis-Pinetum sylvestris and Erico-Pinetum sylvestris.

\begin{tabular}{|c|c|c|c|c|c|c|c|c|c|}
\hline Successive number (Zaporedna številka) & & 1 & 2 & 3 & 4 & 5 & 6 & 7 & 8 \\
\hline Sign for syntaxa (Oznaka sintaksonov) & & RcPs-Si & FPnps & EPs-Do & GPs-CIH & EPsoc-K & EPsoc-A & EPspm & EPsch \\
\hline Authors (Avtorji) & & ARIDTWMZ & LPMV & PP & ID & WF & CEPHSG & CEPHSG & CEPHSG \\
\hline Number of relevés (Število popisov) & & 52 & 11 & 20 & 55 & 19 & 27 & 18 & 36 \\
\hline \multicolumn{10}{|l|}{ Erico-Pinetea } \\
\hline Calamagrostis varia & E1 & 100 & 91 & 60 & 78 & 79 & 59 & 89 & 94 \\
\hline Erica carnea & E1 & 100 & 100 & 100 & 100 & 95 & 96 & 100 & 97 \\
\hline Pinus sylvestris & E3 & 100 & 100 & 100 & 100 & 74 & 100 & 100 & 100 \\
\hline Pinus sylvestris & $\mathrm{E} 2$ & 58 & 18 & 35 & 62 & 16 & 19 & 67 & 50 \\
\hline Polygala chamaebuxus & E1 & 88 & 82 & 30 & 98 & 58 & 82 & 83 & 94 \\
\hline Amelanchier ovalis & E2 & 96 & 91 & 75 & 45 & 53 & 59 & 78 & 94 \\
\hline Epipactis atrorubens & E1 & 71 & 73 & 50 & 60 & . & 30 & 83 & 67 \\
\hline Rhodothamnus chamaecistus & $\mathrm{E} 1$ & 63 & . & 5 & 4 & . & . & . & . \\
\hline Rubus saxatilis & E1 & 60 & 82 & 45 & 2 & 68 & 33 & 67 & 36 \\
\hline Rhododendron hirsutum & E2a & 56 & 27 & 45 & 4 & 26 & 7 & 94 & . \\
\hline Cotoneaster tomentosus & E1 & 50 & 55 & 5 & 4 & 26 & 7 & 39 & 11 \\
\hline Leontodon incanus & E1 & 42 & 18 & 5 & 67 & . & . & 39 & 75 \\
\hline Pinus mugo & $\mathrm{E} 2 \mathrm{~b}$ & 46 & 27 & 50 & 2 & 5 & . & 94 & 3 \\
\hline Euphrasia cuspidata & E1 & 37 & 18 & 35 & 2 & . & . & . & . \\
\hline Carex alba & E1 & 29 & 64 & 40 & 24 & 21 & 59 & 28 & 6 \\
\hline Gymnadenia odoratissima & E1 & 27 & 9 & 35 & 2 & . & 4 & 6 & . \\
\hline Crepis froelichiana subsp. dinarica (C. slovenica) & E1 & 25 & 27 & . & 13 & . & . & . & . \\
\hline Asperula aristata & E1 & 23 & 9 & . & 33 & 5 & . & . & . \\
\hline Chamaecytisus purpureus & E1 & 23 & 64 & 50 & 42 & . & 19 & . & . \\
\hline Arctostaphylos uva-ursi & E1 & 21 & 18 & 40 & . & . & . & . & . \\
\hline Galium austriacum & E1 & 19 & . & . & . & . & . & . & . \\
\hline Cephalanthera rubra & E1 & 17 & 9 & . & 5 & . & . & . & . \\
\hline Allium ericetorum & E1 & 13 & 9 & . & 58 & . & . & . & . \\
\hline Galium purpureum & E1 & 13 & . & 5 & 20 & . & 19 & . & . \\
\hline Aquilegia nigricans & E1 & 8 & . & . & 2 & 16 & . & . & . \\
\hline Genista radiata & $\mathrm{E} 2 \mathrm{a}$ & 8 & 9 & . & 9 & . & . & . & . \\
\hline Molinia caerulea subsp. arundinacea & E1 & 6 & 36 & 35 & 47 & . & 4 & 6 & 11 \\
\hline Aster amellus & E1 & 4 & 18 & . & 15 & . & . & . & . \\
\hline Rhamnus saxatillis & E1 & 4 & 27 & 25 & 16 & 5 & 7 & . & . \\
\hline Polygala nicaeensis subsp. forojulensis & E1 & 4 & 27 & 15 & . & . & . & . & . \\
\hline Carex ornithopoda & E1 & 4 & . & 60 & 2 & . & . & 22 & 39 \\
\hline Genista januensis & $\mathrm{E} 1$ & 4 & . & . & 22 & . & . & . & . \\
\hline Pinus nigra & E3 & 4 & . & . & 18 & . & . & . & . \\
\hline Pinus nigra & E2 & 2 & . & . & 6 & . & . & . & . \\
\hline Daphne cneorum & E2 & 2 & 18 & 5 & . & . & 22 & . & . \\
\hline Euphorbia triflora subsp. kerneri & E1 & 2 & 64 & . & . & . & . & . & . \\
\hline Thesium rostratum & $\mathrm{E} 1$ & . & 36 & . & . & . & . & . & 6 \\
\hline Chamaecytisus hirsutus & E1 & . & 27 & 5 & 40 & 5 & . & . & . \\
\hline Bupleurum ranunculoides & E1 & . & 27 & . & . & . & . & . & . \\
\hline Knautia resmanii & E1 & . & 18 & . & . & . & . & . & . \\
\hline Crepis froelichiana subsp. froelichiana & E1 & . & . & 35 & . & . & . & . & . \\
\hline Peucedanum austriacum & E1 & . & . & . & 7 & 11 & . & . & . \\
\hline
\end{tabular}




\begin{tabular}{|c|c|c|c|c|c|c|c|c|c|}
\hline Successive number (Zaporedna številka) & & 1 & 2 & 3 & 4 & 5 & 6 & 7 & 8 \\
\hline Ophrys insectifera & E1 & . & . & . & 4 & . & . & . & . \\
\hline Viola collina & E1 & . & . & . & . & 16 & . & 6 & 47 \\
\hline Pyrola chlorantha & E1 & . & . & . & . & . & 7 & . & . \\
\hline Aquilegia atrata & E1 & . & . & . & . & . & . & 6 & . \\
\hline Pinus uncinata & E3 & . & . & . & . & . & . & . & 25 \\
\hline Pinus uncinata & E2 & . & . & . & . & . & . & . & 6 \\
\hline \multicolumn{10}{|l|}{ Vaccinio-Piceetea } \\
\hline Picea abies & E3 & 62 & 82 & 60 & 56 & 58 & 89 & 44 & 31 \\
\hline Picea abies & E2 & 88 & 45 & . & 76 & 42 & 56 & 56 & 53 \\
\hline Picea abies & E1 & 38 & . & . & 15 & . & . & . & . \\
\hline Hieracium murorum & E1 & 62 & 45 & 50 & 13 & 16 & 37 & 89 & 11 \\
\hline Vaccinium vitis-idaea & E1 & 58 & 36 & 45 & . & 37 & 22 & 89 & 3 \\
\hline Larix decidua & E3 & 48 & 27 & . & 2 & . & 19 & 11 & 11 \\
\hline Larix decidua & $\mathrm{E} 2$ & 21 & 9 & 35 & 2 & 32 & 4 & . & 6 \\
\hline Vaccinium myrtillus & E1 & 44 & 18 & 15 & . & 37 & 26 & 22 & . \\
\hline Valeriana tripteris & E1 & 33 & 18 & 5 & 2 & 47 & 26 & 11 & 58 \\
\hline Solidago virgaurea & E1 & 21 & 18 & . & 4 & 26 & 26 & 33 & 11 \\
\hline Goodyera repens & E1 & 12 & 36 & 35 & . & . & 15 & 11 & . \\
\hline Melampyrum sylvaticum & E1 & 12 & 9 & 15 & . & . & 4 & 33 & 4 \\
\hline Aposeris foetida & E1 & 12 & 18 & . & . & . & . & . & . \\
\hline Gentiana asclepiadea & E1 & 12 & . & 5 & . & 5 & . & . & . \\
\hline Luzula sylvatica & E1 & 12 & . & . & $\cdot$ & 5 & . & . & . \\
\hline Pyrola minor & E1 & 12 & . & . & 4 & . & . & . & . \\
\hline Clematis alpina & E2a & 10 & 18 & 15 & . & 5 & . & . & . \\
\hline Homogyne sylvestris & E1 & 10 & . & . & 4 & 37 & . & . & . \\
\hline Rosa pendulina & E2a & 8 & . & 5 & 2 & 11 & . & 89 & 3 \\
\hline Gymnocarpium dryopteris & E1 & 8 & . & . & . & . & . & . & . \\
\hline Pyrola rotundifolia & E1 & 6 & 9 & . & . & 11 & . & . & . \\
\hline Listera cordata & E1 & 6 & . & . & . & . & . & . & . \\
\hline Maianthemum bifolium & E1 & 4 & 9 & 5 & . & . & . & . & . \\
\hline Oxalis acetosella & E1 & 4 & $\cdot$ & 5 & . & . & . & . & . \\
\hline Ajuga pyramidalis & E1 & 4 & . & . & . & . & $\cdot$ & . & . \\
\hline Veronica urticifolia & E1 & 2 & 9 & 5 & $\cdot$ & 5 & . & . & . \\
\hline Calamagrostis villosa & E1 & 2 & . & 10 & . & . & . & . & . \\
\hline Abies alba & E3 & 2 & . & 5 & . & . & . & . & . \\
\hline Abies alba & E2 & 2 & . & . & . & 5 & . & . & . \\
\hline Orthilia secunda & E1 & 2 & . & 5 & . & . & 15 & 6 & . \\
\hline Homogyne alpina & E1 & 2 & . & 5 & . & . & . & 33 & . \\
\hline Luzula luzuloides & E1 & 2 & . & 5 & $\cdot$ & . & . & $\cdot$ & . \\
\hline Huperzia selago & E1 & 2 & . & . & . & . & . & 22 & . \\
\hline Diphasiastrum complanatum & E1 & 2 & . & . & . & . & . & . & . \\
\hline Dryopteris dilatata & E1 & 2 & . & . & . & . & . & . & . \\
\hline Pyrola media & E1 & 2 & . & . & . & . & $\cdot$ & $\cdot$ & . \\
\hline Lonicera nigra & E2 & . & 18 & . & . & . & . & . & . \\
\hline Lycopodium annotinum & E1 & . & 9 & . & . & . & . & . & . \\
\hline Calamagrostis arundinacea & E1 & . & 9 & . & . & . & . & . & . \\
\hline Pinus cembra & E3 & . & . & 5 & . & . & . & . & . \\
\hline Avenella flexuosa & E1 & . & . & 5 & . & . & . & . & . \\
\hline Luzula sieberi & E1 & . & . & 5 & . & . & . & . & . \\
\hline Phegopteris connectilis & E1 & . & . & . & . & 5 & . & . & . \\
\hline
\end{tabular}




\begin{tabular}{|c|c|c|c|c|c|c|c|c|c|}
\hline Successive number (Zaporedna številka) & & 1 & 2 & 3 & 4 & 5 & 6 & 7 & 8 \\
\hline \multicolumn{10}{|l|}{ Aremonio-Fagion } \\
\hline Cyclamen purpurascens & E1 & 88 & 73 & 10 & 82 & 68 & 59 & . & 3 \\
\hline Helleborus niger & E1 & 73 & 27 & . & 71 & 26 & 33 & 6 & . \\
\hline Anemone trifolia & E1 & 40 & 64 & 5 & 7 & 63 & 48 & . & . \\
\hline Knautia drymeia & E1 & 13 & . & . & 2 & . & . & . & . \\
\hline Rhamnus fallax & $\mathrm{E} 2 \mathrm{~b}$ & 2 & . & . & 2 & 16 & . & . & . \\
\hline Euphorbia carniolica & E1 & . & . & 15 & 4 & . & . & . & . \\
\hline Omphalodes verna & E1 & . & . & . & 15 & . & . & . & . \\
\hline Primula vulgaris & E1 & . & . & . & 4 & . & . & . & . \\
\hline Daphne laureola & E2 & . & . & . & 2 & . & . & . & . \\
\hline Hemerocallis lilioasphodelus & E1 & . & . & . & 2 & . & . & . & . \\
\hline Tilio-Acerion & & . & . & . & . & . & . & . & . \\
\hline Acer pseudoplatanus & E3 & 2 & . & . & . & 11 & . & . & . \\
\hline Acer pseudoplatanus & $\mathrm{E} 2 \mathrm{~b}$ & 13 & 9 & 5 & 11 & 16 & 19 & 6 & 19 \\
\hline Acer pseudoplatanus & E1 & 33 & . & . & 44 & 42 & . & . & . \\
\hline Juglans regia & $\mathrm{E} 2 \mathrm{a}$ & 2 & 9 & . & 44 & . & . & . & . \\
\hline Acer platanoides & E1 & . & . & . & 7 & . & . & . & . \\
\hline Tilia platyphyllos & E1 & . & . & . & 5 & . & . & . & . \\
\hline Euonymus latifolia & E2 & . & . & . & 4 & 11 & . & . & . \\
\hline Aruncus dioicus & E1 & . & . & . & 2 & . & . & . & . \\
\hline Polytrichum aculeatum & E1 & . & . & . & 2 & . & . & . & . \\
\hline \multicolumn{10}{|l|}{ Alnion incanae, Salicetea purpureae } \\
\hline Frangula alnus & $\mathrm{E} 2 \mathrm{~b}$ & 4 & 27 & 15 & 29 & 5 & 22 & 11 & . \\
\hline Alnus incana & E2 & . & . & 5 & . & 5 & . & . & . \\
\hline Salix eleagnos & E2 & . & . & . & 15 & . & . & . & . \\
\hline Salix purpurea & E2 & . & . & . & 4 & . & . & . & . \\
\hline \multicolumn{10}{|l|}{ Fagetalia sylvaticae } \\
\hline Euphorbia amygdaloides & E1 & 48 & 9 & . & . & 32 & 22 & 6 & . \\
\hline Daphne mezereum & $\mathrm{E} 2 \mathrm{a}$ & 25 & 9 & 10 & 5 & 63 & 19 & 11 & 8 \\
\hline Fagus sylvatica & E3b & 17 & 45 & . & 18 & 63 & 33 & . & 3 \\
\hline Fagus sylvatica & E2 & 33 & 27 & 10 & 27 & . & 11 & . & 6 \\
\hline Fagus sylvatica & E1 & . & . & . & 22 & . & . & . & . \\
\hline Mercurialis perennis & E1 & 23 & 9 & . & 2 & 37 & 4 & 6 & 19 \\
\hline Melica nutans & E1 & 17 & 36 & 5 & 5 & 42 & 37 & 22 & 28 \\
\hline Neottia nidus-avis & E1 & 17 & 9 & . & 15 & 5 & . & . & . \\
\hline Epipactis helleborine & E1 & 15 & 9 & 50 & 16 & 5 & . & 17 & . \\
\hline Prenanthes purpurea & E1 & 13 & 9 & 5 & 4 & 16 & . & . & . \\
\hline Laburnum alpinum & E3 & 4 & . & . & 9 & . & . & . & . \\
\hline Laburnum alpinum & E2 & 17 & . & . & 11 & 16 & . & . & . \\
\hline Luzula nivea & E1 & 12 & 18 & 15 & . & . & . & . & . \\
\hline Viola reichenbachiana & E1 & 10 & 9 & . & 4 & 21 & . & . & . \\
\hline Galium laevigatum & E1 & 8 & . & . & 5 & 16 & . & . & . \\
\hline Lonicera alpigena & E2b & 8 & 27 & 5 & 2 & 36 & . & . & . \\
\hline Salvia glutinosa & E1 & 8 & 9 & . & 15 & 37 & 15 & . & . \\
\hline Cephalanthera damasonium & E1 & 6 & . & . & 9 & . & . & . & . \\
\hline Campanula trachelium & E1 & 4 & . & . & . & . & . & . & . \\
\hline Lilium martagon & E1 & 4 & 9 & 10 & 2 & 5 & . & . & . \\
\hline Brachypodium sylvaticum & E1 & 2 & . & . & . & 5 & . & . & . \\
\hline Euphorbia dulcis & E1 & 2 & . & . & . & . & . & . & . \\
\hline Heracleum sphondylium & E1 & 2 & . & . & . & . & . & . & . \\
\hline
\end{tabular}




\begin{tabular}{|c|c|c|c|c|c|c|c|c|c|}
\hline Successive number (Zaporedna številka) & & 1 & 2 & 3 & 4 & 5 & 6 & 7 & 8 \\
\hline Mycelis muralis & E1 & 2 & . & . & 2 & . & . & . & . \\
\hline Symphytum tuberosum & E1 & 2 & . & . & . & . & . & . & . \\
\hline Carex sylvatica & E1 & . & 9 & 10 & . & . & . & . & . \\
\hline Cypripedium calceolus & E1 & . & . & 20 & . & 5 & . & . & . \\
\hline Prunus avium & E3 & . & . & . & 2 & . & . & . & . \\
\hline Prunus avium & E2 & . & . & . & 2 & . & . & . & . \\
\hline Tilia cordata & E2 & . & . & . & 2 & . & . & . & . \\
\hline Asarum europaeum subsp. caucasicum & E1 & . & . & . & . & 5 & . & . & . \\
\hline Cardamine pentaphyllos & E1 & . & . & . & . & 5 & . & . & . \\
\hline Lathyrus vernus & E1 & . & . & . & . & 5 & . & . & . \\
\hline Fraxinus excelsior & E3 & . & . & . & . & . & 4 & . & \\
\hline Fraxinus excelsior & E2 & . & . & . & . & . & . & . & 3 \\
\hline \multicolumn{10}{|l|}{ Quercetalia pubescenti-petraeae } \\
\hline Sorbus aria & E3 & 23 & . & . & 33 & 79 & 52 & . & 6 \\
\hline Sorbus aria & E2 & 96 & 45 & 25 & 58 & 26 & 37 & 11 & 58 \\
\hline Sorbus aria & E1 & . & . & . & 15 & . & . & . & . \\
\hline Fraxinus ornus & E3 & 4 & 36 & . & 65 & 58 & . & 30 & . \\
\hline Fraxinus ornus & $\mathrm{E} 2$ & 29 & 10 & 15 & 93 & 47 & . & 41 & . \\
\hline Fraxinus ornus & E1 & . & . & . & 47 & . & . & . & . \\
\hline Convallaria majalis & E1 & 21 & 9 & 10 & 9 & 47 & 26 & 6 & 22 \\
\hline Ostrya carpinifolia & E3 & 2 & 18 & . & 84 & 84 & 26 & . & . \\
\hline Ostrya carpinifolia & $\mathrm{E} 2$ & 21 & 18 & 20 & 91 & 47 & 30 & . & . \\
\hline Ostrya carpinifolia & E1 & . & . & . & 13 & . & . & . & . \\
\hline Hypericum montanum & E1 & 8 & . & . & 2 & . & . & . & . \\
\hline Carex flacca & E1 & 4 & . & 10 & 4 & 16 & 15 & 22 & 6 \\
\hline Lathyrus niger & E1 & 4 & . & . & . & . & . & . & . \\
\hline Melittis melissophyllum & E1 & 2 & 9 & . & 9 & 5 & . & . & . \\
\hline Epipactis muelleri & E1 & 2 & . & . & 5 & . & . & . & . \\
\hline Coronila emeroides & E2 & . & 18 & . & . & . & . & . & . \\
\hline Mercurialis ovata & E1 & . & 18 & . & 25 & . & . & . & . \\
\hline Prunus mahaleb & E2 & . & 9 & . & . & . & . & . & . \\
\hline Coronilla emerus & E2 & . & . & 5 & 11 & . & . & . & . \\
\hline Tamus communis & E1 & . & . & 5 & . & . & . & . & . \\
\hline Cornus mas & E2 & . & . & . & 7 & . & . & . & . \\
\hline Euonymus verrucosa & E2 & . & . & . & 7 & . & . & . & . \\
\hline Quercus pubescens & E3 & . & . & . & 6 & . & . & . & . \\
\hline Sesleria autumnalis & E1 & . & . & . & 5 & . & . & . & . \\
\hline Asparagus tenuifolius & E1 & . & . & . & 2 & . & . & . & . \\
\hline Cotinus coggygria & E2 & . & . & . & 2 & . & . & . & . \\
\hline Peucedanum schottii & E2 & . & . & . & 2 & . & . & . & . \\
\hline Quercus pubescens & E2 & . & . & . & 2 & . & . & . & . \\
\hline Clematis recta & E2 & . & . & . & . & 5 & . & . & . \\
\hline \multicolumn{10}{|l|}{ Quercetalia roboris-petraeae } \\
\hline Pteridium aquilinum & E1 & 42 & 45 & 10 & 15 & 26 & 41 & 6 & . \\
\hline Melampyrum pratense & E1 & 23 & 18 & 5 & . & 26 & 30 & 89 & . \\
\hline Potentilla erecta & E1 & 21 & 82 & 30 & 15 & . & 11 & 22 & 19 \\
\hline Populus tremula & E1 & 2 & . & . & . & . & . & . & . \\
\hline Lembotropis nigricans & E2 & . & 36 & 10 & . & 5 & . & . & . \\
\hline Betula pubescens & E2 & . & 9 & . & . & 16 & . & . & . \\
\hline Quercus petraeae & E2 & . & 9 & . & 36 & 11 & . & . & . \\
\hline
\end{tabular}




\begin{tabular}{|c|c|c|c|c|c|c|c|c|c|}
\hline Successive number (Zaporedna številka) & & 1 & 2 & 3 & 4 & 5 & 6 & 7 & 8 \\
\hline Betula pendula & E3 & . & . & . & 4 & . & . & . & . \\
\hline Betula pendula & E2 & . & 9 & . & 5 & 5 & . & . & . \\
\hline Betonica officinalis & E1 & . & . & 5 & 4 & . & . & . & . \\
\hline Quercus robur & E3 & . & . & . & . & . & 4 & . & . \\
\hline Quercus robur & $\mathrm{E} 2$ & . & . & . & 13 & 5 & 15 & . & . \\
\hline Populus tremula & E3 & . & . & . & 4 & . & . & . & . \\
\hline Populus tremula & E2 & . & . & . & 7 & . & . & . & . \\
\hline Hieracium sabaudum & E1 & . & . & . & 4 & . & . & . & . \\
\hline Alnus glutinosa & E3 & . & . & . & 2 & . & . & . & . \\
\hline Castanea sativa & $\mathrm{E} 1$ & . & . & . & 4 & . & . & . & . \\
\hline Hieracium racemosum & E1 & . & . & . & 2 & . & . & . & . \\
\hline Chamaecytisus supinus & E2 & . & . & . & . & 11 & . & . & . \\
\hline Chamaespartium sagittale & E1 & . & . & . & . & 5 & . & . & . \\
\hline \multicolumn{10}{|l|}{ Querco-Fagetea } \\
\hline Platanthera bifolia & E1 & 42 & 45 & 20 & 7 & 16 & 56 & 6 & . \\
\hline Carex digitata & E1 & 13 & 18 & . & 16 & . & . & . & . \\
\hline Hepatica nobilis & E1 & 10 & 18 & 15 & 2 & 32 & . & $\cdot$ & . \\
\hline Cephalanthera longifolia & E1 & 8 & . & . & 7 & . & . & . & . \\
\hline Corylus avellana & E1 & 6 & 18 & 15 & 24 & . & 4 & 6 & . \\
\hline Cruciata glabra & E1 & 6 & 27 & 5 & 4 & . & . & . & . \\
\hline Dactylorhiza fuchsii & E1 & 4 & . & 10 & . & 16 & . & . & . \\
\hline Pyrus pyraster & E3 & . & . & . & 2 & . & . & . & . \\
\hline Pyrus pyraster & E2 & 4 & . & 5 & 2 & . & . & . & . \\
\hline Anemone nemorosa & E1 & 2 & . & 5 & . & . & . & . & . \\
\hline Festuca heterophylla & E1 & 2 & . & . & . & . & . & . & . \\
\hline Listera ovata & E1 & 2 & . & 35 & 18 & . & . & . & . \\
\hline Taxus baccata & E3a & 2 & . & . & 2 & . & . & . & . \\
\hline Taxus baccata & E2 & . & . & . & 2 & . & . & . & . \\
\hline Lonicera xylosteum & E2 & . & 9 & 15 & 2 & 32 & 15 & $\cdot$ & . \\
\hline Carex montana & E1 & . & 9 & . & . & . & . & 11 & 8 \\
\hline Viola mirabilis & $\mathrm{E} 1$ & . & 9 & . & . & . & . & . & . \\
\hline Hedera helix & E1 & . & . & . & 20 & . & . & . & . \\
\hline Clematis vitalba & E2 & . & . & . & 16 & 16 & . & . & . \\
\hline Rosa arvensis & E2 & . & . & . & 5 & . & . & . & . \\
\hline Viscum album subsp. album & E3 & . & . & . & 5 & . & . & . & . \\
\hline Malus sylvestris & E2 & . & . & . & 4 & . & . & . & . \\
\hline Veratrum nigrum & E1 & . & . & . & 4 & . & . & . & . \\
\hline Vinca minor & E1 & . & . & . & 4 & . & . & . & . \\
\hline Acer campestre & E1 & . & . & . & 2 & . & . & . & . \\
\hline Ilex aquifolium & E2 & . & . & . & 2 & . & . & . & . \\
\hline \multicolumn{10}{|l|}{ Sambuco-Salicion capreae } \\
\hline Sorbus aucuparia & E3 & 10 & 45 & . & . & 11 & $\cdot$ & $\cdot$ & . \\
\hline Sorbus aucuparia & E2 & 40 & 18 & 10 & 2 & 32 & 15 & 11 & 39 \\
\hline Salix caprea & $\mathrm{E} 2 \mathrm{~b}$ & 2 & 9 & 10 & 4 & . & . & . & . \\
\hline \multicolumn{10}{|l|}{ Rhamno-Prunetea } \\
\hline Juniperus communis & $\mathrm{E} 2 \mathrm{~b}$ & 50 & 73 & 75 & 93 & 5 & 37 & 83 & 8 \\
\hline Berberis vulgaris & $\mathrm{E} 2 \mathrm{~b}$ & 8 & 18 & 20 & 40 & 32 & 63 & 28 & 14 \\
\hline Viburnum lantana & $\mathrm{E} 2 \mathrm{~b}$ & 6 & 36 & 10 & 42 & 32 & 33 & . & . \\
\hline Rhamnus catharticus & $\mathrm{E} 2 \mathrm{~b}$ & 2 & . & . & 31 & . & 15 & . & 3 \\
\hline Cotoneaster integerrimus & E2 & . & . & 15 & . & . & . & . & . \\
\hline
\end{tabular}




\begin{tabular}{|c|c|c|c|c|c|c|c|c|c|}
\hline Successive number (Zaporedna številka) & & 1 & 2 & 3 & 4 & 5 & 6 & 7 & 8 \\
\hline Ligustrum vulgare & $\mathrm{E} 2$ & . & . & . & 16 & 5 & 15 & . & . \\
\hline Crataegus monogyna & E2 & . & . & . & 9 & . & 30 & . & . \\
\hline Cornus sanguinea & E2 & . & . & . & 9 & . & . & . & . \\
\hline Rosa canina & E2 & . & . & . & 7 & . & . & . & . \\
\hline Rubus fruticosus agg. & E2 & . & . & . & 4 & . & . & . & . \\
\hline Euonymus europaea & E2 & . & . & . & 2 & . & . & . & . \\
\hline Rubus montanus & E2 & . & . & . & 2 & . & . & . & . \\
\hline \multicolumn{10}{|l|}{ Trifolio-Geranietea } \\
\hline Viola hirta & E1 & 35 & 36 & 25 & 33 & . & . & . & 11 \\
\hline Vincetoxicum hirundinaria & E1 & 27 & 36 & 5 & 51 & 16 & 30 & 11 & 56 \\
\hline Laserpitium siler & E1 & 17 & 9 & 30 & 9 & . & . & . & . \\
\hline Polygonatum odoratum & E1 & 17 & 18 & 25 & 15 & 16 & 15 & 6 & 39 \\
\hline Anthericum ramosum & E1 & 15 & 36 & 15 & 40 & . & 7 & 11 & 56 \\
\hline Digitalis grandiflora & E1 & 10 & . & 10 & 4 & 5 & . & . & . \\
\hline Laserpitium latifolium & E1 & 6 & 9 & 15 & . & 5 & . & 11 & 28 \\
\hline Clinopodium vulgare & E1 & 6 & 9 & . & 2 & . & . & . & . \\
\hline Origanum vulgare & E1 & 6 & . & . & 15 & . & . & . & . \\
\hline Vicia incana & E1 & 4 & . & . & . & . & . & . & . \\
\hline Thalictrum minus s. lat. & E1 & 2 & . & 5 & 18 & . & . & . & . \\
\hline Astragalus glycyphyllos & E1 & 2 & . & . & . & . & . & . & . \\
\hline Iris graminea & E1 & 2 & . & . & 2 & . & . & . & . \\
\hline Trifolium rubens & E1 & 2 & . & . & . & . & . & . & . \\
\hline Melampyrum nemorosum & E1 & . & . & 5 & . & . & . & . & . \\
\hline Salvia pratensis subsp. saccardiana & E1 & . & . & . & 27 & . & . & . & . \\
\hline Thesium bavarum & E1 & . & . & . & 9 & . & 11 & . & . \\
\hline Geranium sanguineum & E1 & . & . & . & 5 & 5 & . & . & . \\
\hline Calamintha einseleana & E1 & . & . & . & 4 & . & . & . & . \\
\hline Grafia golaka & E1 & . & . & . & 4 & . & . & . & . \\
\hline Verbascum austriacum & E1 & . & . & . & 4 & . & . & . & . \\
\hline Silene nutans & E1 & . & . & . & 2 & 5 & . & . & . \\
\hline Lilium carniolicum & $\mathrm{E} 1$ & . & . & . & 2 & . & . & . & . \\
\hline Peucedanum cervaria & E1 & . & . & . & 2 & . & . & . & . \\
\hline \multicolumn{10}{|l|}{ Betulo-Alnetea } \\
\hline Salix glabra & $\mathrm{E} 2 \mathrm{a}$ & 35 & 27 & 45 & 15 & 5 & . & . & . \\
\hline Salix appendiculata & E2 & 12 & 9 & 5 & 24 & . & . & . & . \\
\hline Salix waldsteiniana & E2a & 4 & . & 5 & . & . & . & . & . \\
\hline Sorbus chamaemespilus & E2 & . & 27 & 20 & . & . & . & 83 & . \\
\hline Salix hastata & E2 & . & . & 35 & . & . & . & . & . \\
\hline Juniperus sibirica & $\mathrm{E} 2$ & . & . & 5 & . & . & . & $\cdot$ & . \\
\hline \multicolumn{10}{|l|}{ Mulgedio-Aconitetea } \\
\hline Silene vulgaris subsp. antelopum & E1 & 8 & . & . & . & . & . & . & . \\
\hline Phyteuma ovatum & E1 & 6 & . & . & . & . & . & . & . \\
\hline Polygonatum verticillatum & E1 & 4 & . & . & . & . & . & . & . \\
\hline Thalictrum aquilegiifolium & E1 & 4 & . & . & . & . & . & . & . \\
\hline Ranunculus platanifolius & E1 & 2 & . & . & . & . & . & . & . \\
\hline Lathyrus laevigatus & E1 & . & . & 5 & . & . & . & . & . \\
\hline Aconitum lycoctonum subsp. ranunculifolium & E1 & . & . & . & . & 5 & . & . & . \\
\hline Knautia dipsacifolia (K. maxima) & E1 & . & . & . & . & . & . & 6 & 8 \\
\hline \multicolumn{10}{|l|}{ Epilobietea angustifolii } \\
\hline Fragaria vesca & E1 & 12 & 18 & 10 & . & . & 15 & 6 & 25 \\
\hline
\end{tabular}




\begin{tabular}{|c|c|c|c|c|c|c|c|c|c|}
\hline Successive number (Zaporedna številka) & & 1 & 2 & 3 & 4 & 5 & 6 & 7 & 8 \\
\hline Potentilla recta & E1 & 2 & . & & . & 5 & . & . & . \\
\hline Chamerion angustifolium & E1 & 2 & . & . & . & . & . & . & . \\
\hline Lysimachia vulgaris & E1 & . & . & 5 & . & . & . & . & . \\
\hline Bromus ramosus & E1 & . & . & 5 & . & . & . & . & . \\
\hline Silene alba & E1 & . & . & 5 & . & . & . & . & . \\
\hline Atropa belladona & E1 & . & . & . & 2 & . & . & . & . \\
\hline Picris hieracioides & E1 & . & . & . & 2 & . & . & . & . \\
\hline Vrebascum thapsus & $\mathrm{E} 2$ & . & . & . & 2 & . & . & . & . \\
\hline \multicolumn{10}{|l|}{ Elyno-Seslerietea } \\
\hline Betonica alopecuros & E1 & 77 & 82 & 55 & 38 & 16 & . & . & . \\
\hline Sesleria caerulea & E1 & 67 & 64 & 80 & 84 & 16 & 26 & 89 & 72 \\
\hline Laserpitium peucedanoides & E1 & 65 & . & 30 & . & 11 & . & . & . \\
\hline Thymus praecox subsp. polytrichus & $\mathrm{E} 1$ & 52 & . & 20 & . & . & . & . & . \\
\hline Globularia cordifolia & E1 & 50 & 27 & 60 & 44 & . & 11 & 22 & 47 \\
\hline Carex mucronata & E1 & 37 & . & . & 33 & . & . & . & 50 \\
\hline Scabiosa lucida subsp. lucida & E1 & 35 & . & 20 & . & . & . & . & 36 \\
\hline Phyteuma orbiculare & E1 & 29 & . & 15 & 9 & . & . & 33 & 17 \\
\hline Senecio abrotanifolius & E1 & 21 & 9 & . & . & . & . & . & . \\
\hline Aster bellidiastrum & E1 & 19 & . & 5 & 2 & . & . & 22 & . \\
\hline Euphrasia salisburgensis & E1 & 17 & . & 5 & 9 & . & . & 83 & 22 \\
\hline Dryas octopetala & E1 & 15 & . & 35 & . & . & . & . & 3 \\
\hline Acinos alpinus & E1 & 13 & 27 & 5 & . & . & . & . & 8 \\
\hline Carduus crassifolius & E1 & 13 & . & . & . & . & . & . & . \\
\hline Campanula witasekiana & E1 & 12 & . & . & . & . & . & . & . \\
\hline Gentiana clusii & E1 & 12 & . & 15 & . & . & . & . & . \\
\hline Carduus defloratus & E1 & 8 & 18 & 30 & . & . & . & 61 & 92 \\
\hline Thesium alpinum & E1 & 8 & . & 10 & . & . & . & 28 & 33 \\
\hline Carex firma & E1 & 6 & . & . & . & . & . & . & . \\
\hline Juncus monanthos & E1 & 6 & . & . & . & . & . & . & . \\
\hline Rhinanthus glacialis & E1 & 4 & . & 15 & . & . & . & . & . \\
\hline Globularia nudicaulis & E1 & 4 & . & . & . & . & . & 6 & . \\
\hline Helianthemum nummularium subsp. grandiflorum & E1 & 2 & . & 20 & . & . & . & . & . \\
\hline Galium anisophyllon & E1 & 2 & . & 10 & . & . & . & 61 & 75 \\
\hline Helianthemum alpestre & E1 & 2 & . & . & . & . & . & . & . \\
\hline Linum julicum & E1 & 2 & . & . & . & . & . & . & . \\
\hline Polygala alpestris & E1 & 2 & . & . & . & . & . & . & . \\
\hline Scabiosa lucida subsp. stricta & E1 & 2 & . & . & . & . & . & . & . \\
\hline Leucanthemum maximum (L. heterophyllum) & E1 & . & 9 & 30 & . & . & . & . & . \\
\hline Anthylis vulneraria subsp. alpestris & E1 & . & . & 20 & . & . & . & . & $\cdot$ \\
\hline Carex sempervirens & E1 & . & . & 15 & . & . & . & . & 22 \\
\hline Lotus alpinus & E1 & . & . & 15 & . & . & . & . & . \\
\hline Gentianella anisodonta & E1 & . & . & 10 & . & . & . & . & . \\
\hline Horminum pyrenaicum & E1 & . & . & 10 & . & . & . & . & . \\
\hline Pulsatilla alpina & E1 & . & . & 10 & . & . & . & . & . \\
\hline Daphne striata & E1 & . & . & 5 & . & . & . & 78 & 14 \\
\hline Carex ferruginea & E1 & . & . & 5 & . & . & . & 44 & . \\
\hline Selaginella selaginoides & E1 & . & . & 5 & . & . & . & 39 & . \\
\hline Oxytropis jacquinii & E1 & . & . & 5 & . & . & . & . & . \\
\hline Astragalus alpinus & E1 & . & . & 5 & . & . & . & . & . \\
\hline Aster alpinus & E1 & . & . & 5 & . & . & . & . & . \\
\hline
\end{tabular}




\begin{tabular}{|c|c|c|c|c|c|c|c|c|c|}
\hline Successive number (Zaporedna številka) & & 1 & 2 & 3 & 4 & 5 & 6 & 7 & 8 \\
\hline Gentiana nivalis & E1 & . & . & 5 & . & . & . & . & . \\
\hline Knautia longifolia & E1 & . & . & 5 & . & . & . & . & . \\
\hline Festuca alpestris & E1 & . & . & 5 & . & . & . & . & . \\
\hline Pedicularis elongata & E1 & . & . & 5 & . & . & . & . & . \\
\hline Traunsteinera globosa & E1 & . & . & 5 & . & . & . & . & . \\
\hline \multicolumn{10}{|l|}{ Juncetea trifidi } \\
\hline Juncus trifidus & E1 & 2 & . & . & . & . & . & . & . \\
\hline Campanula scheuchzeri & E1 & 2 & . & . & . & . & . & . & . \\
\hline Solidago virgaurea subsp. minuta & E1 & . & . & . & . & 11 & . & . & . \\
\hline \multicolumn{10}{|l|}{ Caricetalia davallianae } \\
\hline Tofieldia calyculata & E1 & 10 & 9 & 20 & 9 & 5 & . & . & . \\
\hline Pinguicula alpina & E1 & 8 & . & . & 7 & . & . & . & . \\
\hline Parnassia palustris & E1 & . & 9 & 20 & 5 & . & . & . & . \\
\hline Malaxis monophyllos & E1 & . & . & 5 & . & . & $\cdot$ & . & . \\
\hline Schoenus nigricans & E1 & . & . & . & 2 & . & . & . & . \\
\hline \multicolumn{10}{|l|}{ Calluno-Ulicetea } \\
\hline Calluna vulgaris & E1 & 6 & 18 & 5 & . & 5 & . & 6 & 3 \\
\hline Antennaria dioica & E1 & 2 & 9 & 10 & . & . & . & . & . \\
\hline Danthonia decumbens & E1 & . & . & . & . & . & 4 & . & . \\
\hline Euphrasia officinalis & E1 & . & . & . & . & . & . & . & 3 \\
\hline \multicolumn{10}{|l|}{ Festuco-Brometea } \\
\hline Buphthalmum salicifolium & E1 & 79 & 73 & 55 & 75 & 58 & 59 & 39 & 97 \\
\hline Carex humilis & E1 & 58 & 36 & 65 & 80 & . & 4 & 78 & 94 \\
\hline Carlina acaulis & E1 & 46 & 9 & 20 & 18 & . & 15 & 56 & 33 \\
\hline Peucedanum oreoselinum & E1 & 44 & 45 & 35 & 31 & 21 & 37 & . & . \\
\hline Gentianella ciliata & E1 & 35 & . & . & 11 & 5 & . & $\cdot$ & . \\
\hline Brachypodium rupestre (incl. B. caespitosum) & E1 & 33 & 82 & 20 & 51 & 21 & 52 & 44 & . \\
\hline Linum catharticum & E1 & 33 & 9 & 5 & 5 & . & . & . & 17 \\
\hline Cirsium erisithales & E1 & 29 & 9 & 10 & 24 & 37 & 15 & . & . \\
\hline Euphorbia cyparissias & E1 & 25 & 9 & 5 & 38 & . & 41 & 11 & . \\
\hline Teucrium chamaedrys & E1 & 25 & 36 & 5 & 64 & 5 & 15 & 6 & 3 \\
\hline Teucrium montanum & E1 & 25 & 36 & 45 & 38 & . & 7 & 17 & 67 \\
\hline Hippocrepis comosa & E1 & 21 & 45 & 25 & 7 & . & 15 & 50 & 36 \\
\hline Gymnadenia conopsea & E1 & 19 & 45 & 5 & 2 & . & . & 17 & 3 \\
\hline Galium lucidum & E1 & 17 & 27 & 15 & 62 & 5 & 7 & 28 & . \\
\hline Dorycnium germanicum & E1 & 15 & . & 5 & 5 & . & . & . & . \\
\hline Galium verum & E1 & 15 & 55 & 35 & 24 & . & 30 & 6 & 8 \\
\hline Stachys recta (incl. S. labiosa) & E1 & 13 & . & 20 & 11 & 5 & . & . & . \\
\hline Prunella grandiflora & E1 & 10 & 45 & 35 & 4 & . & 7 & 17 & 25 \\
\hline Pimpinella saxifraga & E1 & 10 & 18 & 5 & 2 & . & 19 & . & 19 \\
\hline Cirsium pannonicum & E1 & 8 & . & . & 11 & . & . & . & . \\
\hline Coronilla vaginalis & E1 & 4 & 9 & 35 & . & . & 19 & 11 & 17 \\
\hline Genista germanica & E1 & 4 & 27 & . & . & $\cdot$ & $\cdot$ & $\cdot$ & . \\
\hline Genista tinctoria & E1 & 2 & 18 & . & 4 & 5 & . & . & . \\
\hline Asperula cynanchica & E1 & 2 & 9 & . & 11 & . & . & . & . \\
\hline Cirsium acaule & E1 & 2 & . & 5 & . & . & 4 & 6 & . \\
\hline Thymus praecox subsp. praecox & E1 & 2 & . & . & 35 & . & . & 72 & 81 \\
\hline Bromopsis erecta & E1 & 2 & . & . & 15 & . & . & . & . \\
\hline Carlina vulgaris & E1 & 2 & . & . & 5 & . & . & . & . \\
\hline Euphrasia kerneri & E1 & 2 & . & . & . & . & . & . & . \\
\hline
\end{tabular}




\begin{tabular}{|c|c|c|c|c|c|c|c|c|c|}
\hline Successive number (Zaporedna številka) & & 1 & 2 & 3 & 4 & 5 & 6 & 7 & 8 \\
\hline Ophrys sphegodes & E1 & 2 & . & . & . & . & $\cdot$ & . & . \\
\hline Orobanche teucrii & $\mathrm{E} 1$ & 2 & . & . & . & . & . & . & . \\
\hline Plantago media & E1 & 2 & . & . & $\cdot$ & . & $\cdot$ & . & . \\
\hline Sanguisorba minor & E1 & 2 & . & . & . & . & . & . & . \\
\hline Thymus longicaulis & E1 & . & 36 & . & . & . & . & . & . \\
\hline Trifolium montanum & E1 & . & 9 & 5 & . & . & . & . & . \\
\hline Helianthemum ovatum & E1 & . & 9 & . & 5 & . & 4 & . & 6 \\
\hline Centaurea bracteata & E1 & . & 9 & . & 20 & . & . & . & . \\
\hline Scabiosa graminifolia & E1 & . & 9 & . & . & . & . & . & . \\
\hline Allium carinatum subsp. carinatum & E1 & . & 9 & . & 2 & . & $\cdot$ & . & $\cdot$ \\
\hline Betonica serotina & E1 & . & 9 & . & 7 & . & . & . & . \\
\hline Galium pumilum & E1 & . & . & 25 & . & . & . & . & . \\
\hline Hierochloe australis & E1 & . & . & 25 & . & . & . & . & $\cdot$ \\
\hline Koeleria pyramidata & E1 & . & . & 15 & 5 & . & . & . & $\cdot$ \\
\hline Thymus pulegioides s. lat. & E1 & . & . & 10 & . & . & . & . & . \\
\hline Centaurea scabiosa (incl. subsp. fritschii) & E1 & . & . & 5 & 2 & . & 4 & 11 & . \\
\hline Festuca amethystina & E1 & . & . & 5 & . & . & . & . & 6 \\
\hline Ophrys aranifera (O. araneola) & E1 & . & . & 5 & . & . & $\cdot$ & . & $\cdot$ \\
\hline Peucedanum cervaria & E1 & . & . & 5 & $\cdot$ & . & $\cdot$ & . & $\cdot$ \\
\hline Campanula spicata & E1 & . & . & 5 & . & . & . & . & . \\
\hline Ononis spinosa & E1 & . & . & 5 & . & . & $\cdot$ & . & $\cdot$ \\
\hline Gentianella germanica & E1 & . & . & 5 & . & . & $\cdot$ & . & $\cdot$ \\
\hline Acinos arvensis & E1 & . & . & 5 & . & . & $\cdot$ & . & $\cdot$ \\
\hline Campanula rotundifolia agg. & E1 & . & . & . & . & . & 4 & 28 & . \\
\hline Satureja montana subsp. variegata & E1 & . & . & . & 18 & . & . & . & . \\
\hline Scabiosa hladnikiana & E1 & . & . & . & 18 & . & . & . & $\cdot$ \\
\hline Dianthus hyssopifolius & E1 & . & . & . & 13 & . & . & . & $\cdot$ \\
\hline Thesium linophyllon & E1 & . & . & . & 9 & . & . & . & . \\
\hline Allium carinatum subsp. pulchellum & E1 & . & . & . & 7 & . & $\cdot$ & . & $\cdot$ \\
\hline Campanula glomerata & E1 & . & . & . & 7 & . & . & . & $\cdot$ \\
\hline Silene vulgaris subsp. vulgaris & E1 & . & . & . & 6 & . & . & . & $\cdot$ \\
\hline Scabiosa triandra & E1 & . & . & . & 5 & . & $\cdot$ & . & $\cdot$ \\
\hline Carduus nutans & E1 & . & . & . & 4 & . & $\cdot$ & . & $\cdot$ \\
\hline Dorycnium herbaceum & E1 & . & . & . & 4 & . & . & . & $\cdot$ \\
\hline Hieracium pilosella & E1 & . & . & . & 4 & . & . & . & . \\
\hline Veronica barrelieri & E1 & . & . & . & 4 & . & $\cdot$ & . & $\cdot$ \\
\hline Centaurium erythraea & E1 & . & . & . & 2 & . & . & . & . \\
\hline Cirsium $x$ linkianum & E1 & . & . & . & 2 & . & . & . & . \\
\hline Danthonia alpina & E1 & . & . & . & 2 & . & $\cdot$ & . & . \\
\hline Genista ovata & E1 & . & . & . & 2 & . & $\cdot$ & . & . \\
\hline Globularia punctata & E1 & . & . & . & 2 & . & . & . & . \\
\hline Hieracium bauhinii & E1 & $\cdot$ & . & . & 2 & . & $\cdot$ & . & . \\
\hline Inula hirta & E1 & . & . & . & 2 & . & $\cdot$ & . & . \\
\hline Potentilla pusilla & E1 & . & . & . & 2 & . & $\cdot$ & . & $\cdot$ \\
\hline \multicolumn{10}{|l|}{ Poo alpinae-Trisetetalia } \\
\hline Paradisea liliastrum & E1 & . & . & 5 & . & . & $\cdot$ & . & . \\
\hline Ranunculus nemorosus agg. & E1 & . & . & . & 6 & . & $\cdot$ & 11 & 11 \\
\hline \multicolumn{10}{|l|}{ Molinio-Arrbenatheretea, Molinion } \\
\hline Lotus corniculatus & E1 & 67 & 45 & 60 & 24 & . & 19 & 72 & 33 \\
\hline Lathyrus pratensis & E1 & 23 & 27 & 5 & . & 5 & 19 & . & . \\
\hline
\end{tabular}




\begin{tabular}{|c|c|c|c|c|c|c|c|c|c|}
\hline Successive number (Zaporedna številka) & & 1 & 2 & 3 & 4 & 5 & 6 & 7 & 8 \\
\hline Galium mollugo & E1 & 8 & 9 & . & . & . & 7 & . & 22 \\
\hline Vicia cracca & E1 & 6 & . & . & . & . & . & . & . \\
\hline Vicia sepium & E1 & 4 & . & . & . & . & . & . & . \\
\hline Galium album & E1 & 2 & 18 & . & . & . & . & . & . \\
\hline Leontodon hispidus & E1 & 2 & . & 5 & . & $\cdot$ & 19 & 17 & 25 \\
\hline Veronica chamaedrys & E1 & 2 & . & . & . & 5 & . & . & . \\
\hline Succisa pratensis & E1 & 2 & . & . & . & . & . & . & . \\
\hline Leucanthemum ircutianum & E1 & 2 & . & . & . & . & . & . & . \\
\hline Plantago lanceolata & E1 & 2 & . & . & . & . & . & . & . \\
\hline Centaurea nigrescens & E1 & . & . & 5 & . & 11 & . & . & . \\
\hline Dactylis glomerata & E1 & . & . & 5 & 5 & . & . & . & . \\
\hline Pimpinella major & E1 & . & . & 5 & . & . & . & . & . \\
\hline Festuca rubra & E1 & . & . & 5 & . & . & . & . & . \\
\hline Pastinaca sativa & E1 & . & . & 5 & . & . & $\cdot$ & . & . \\
\hline Achillea millefolium & E1 & . & . & . & 2 & . & . & . & . \\
\hline Taraxacum officinale agg. & E1 & . & . & . & 2 & . & . & . & . \\
\hline Centaurea jacea & E1 & . & . & . & . & . & 4 & . & . \\
\hline Galium boreale & E1 & . & . & . & . & . & . & . & 6 \\
\hline \multicolumn{10}{|l|}{ Thlaspietea rotundifolii } \\
\hline Biscutella laevigata & E1 & 48 & 27 & 30 & 42 & . & 4 & 11 & 3 \\
\hline Hieracium porrifolium & E1 & 48 & 18 & . & 38 & . & . & . & . \\
\hline Gymnocarpium robertianum & E1 & 37 & . & 15 & 2 & 26 & . & . & . \\
\hline Hieracium bifidum & E1 & 25 & . & 10 & 22 & 16 & 4 & 11 & 6 \\
\hline Heliosperma alpestre & E1 & 13 & . & . & . & . & . & . & . \\
\hline Achnatherum calamagrostis & E1 & 12 & 9 & 10 & 33 & . & 7 & . & . \\
\hline Petasites paradoxus & E1 & 4 & . & 25 & 9 & 47 & 22 & 17 & 3 \\
\hline Rumex scutatus & E1 & 4 & . & 10 & . & . & . & . & . \\
\hline Hieracium glaucum & E1 & 4 & . & 5 & 36 & . & . & 22 & 44 \\
\hline Adenostyles glabra & E1 & 4 & . & . & . & 5 & . & . & . \\
\hline Silene vulgaris subsp. glareosa & E1 & 2 & 9 & 5 & 5 & . & . & . & . \\
\hline Valeriana montana & E1 & 2 & . & 25 & . & . & . & 39 & . \\
\hline Gypsophila repens & E1 & 2 & . & 5 & . & . & . & . & . \\
\hline Astrantia carniolica & E1 & 2 & . & . & . & . & . & . & . \\
\hline Trisetum argenteum & E1 & 2 & . & . & . & . & . & . & . \\
\hline Aquilegia einseleana & E1 & . & 27 & 25 & . & . & . & . & . \\
\hline Hieracium bupleuroides & E1 & . & . & 30 & . & . & . & . & . \\
\hline Trisetum argenteum & E1 & . & . & 20 & . & . & . & . & . \\
\hline Athamantha cretensis & E1 & . & . & 15 & . & . & . & . & . \\
\hline Dianthus strenbergii & E1 & . & . & 15 & . & . & . & . & . \\
\hline Festuca spectabilis & E1 & . & . & 10 & . & . & . & . & . \\
\hline Galium margaritaceum & E1 & . & . & 10 & . & . & . & . & . \\
\hline Saxifraga caesia & E1 & . & . & 5 & . & . & . & . & . \\
\hline Hieracium piloselloides & E1 & . & . & 5 & . & . & . & . & . \\
\hline Peucedanum verticillare & E1 & . & . & . & 7 & 16 & . & . & . \\
\hline Chamaenerion palustre & E1 & . & . & . & 5 & . & . & . & . \\
\hline Hieracium dollineri & E1 & . & . & . & 4 & . & . & . & . \\
\hline Hieracium schmidtii & E1 & . & . & . & 4 & . & . & . & . \\
\hline Hieracium caesium & E1 & . & . & . & 2 & . & . & . & . \\
\hline Petrorhagia saxifraga & E1 & . & . & . & 2 & . & . & . & . \\
\hline Scrophularia juratensis & E1 & . & . & . & 2 & . & . & . & . \\
\hline
\end{tabular}




\begin{tabular}{|c|c|c|c|c|c|c|c|c|c|}
\hline Successive number (Zaporedna številka) & & 1 & 2 & 3 & 4 & 5 & 6 & 7 & 8 \\
\hline Orobanche flava & E1 & . & . & . & . & 5 & . & . & . \\
\hline \multicolumn{10}{|c|}{ Potentilletalia caulescentis, Physoplexido-Saxifragion } \\
\hline Valeriana saxatilis & E1 & 54 & 9 & 15 & 9 & 11 & . & . & . \\
\hline Campanula cespitosa & $\mathrm{E} 1$ & 52 & 55 & 25 & 75 & 5 & . & . & . \\
\hline Potentilla caulescens & E1 & 31 & . & . & 5 & . & . & . & 47 \\
\hline Primula auricula & $\mathrm{E} 1$ & 27 & . & 5 & . & . & . & 6 & 78 \\
\hline Campanula cochleariifolia & E1 & 12 & . & 5 & . & . & . & 28 & 72 \\
\hline Silene hayekiana & $\mathrm{E} 1$ & 10 & . & . & 9 & . & . & . & . \\
\hline Rhamnus pumilus & E1 & 8 & . & . & . & . & . & 28 & 53 \\
\hline Saxifraga squarrosa & E1 & 4 & . & 5 & . & . & . & . & . \\
\hline Silene saxifraga & E1 & 2 & . & . & 9 & . & . & . & . \\
\hline Paederota lutea & E1 & 2 & . & . & . & . & . & . & . \\
\hline Spireae decumbens & E1 & . & 9 & . & . & . & . & . & . \\
\hline Seseli gouanii & E1 & . & & 10 & . & . & . & . & . \\
\hline Phyteuma scheuchzeri subsp. columnae & E1 & . & . & . & 33 & . & . & . & . \\
\hline Primula carniolica & $\mathrm{E} 1$ & . & . & . & 11 & . & . & . & . \\
\hline Athamantha turbith & $\mathrm{E} 1$ & . & . & . & 2 & . & . & . & . \\
\hline Hieracium pospichalii & $\mathrm{E} 1$ & . & . & . & 2 & . & . & . & . \\
\hline Heliosperma pusillum & $\mathrm{E} 1$ & . & . & . & . & 5 & . & . & . \\
\hline \multicolumn{10}{|l|}{ Asplenietea trichomanis } \\
\hline Asplenium ruta-muraria & $\mathrm{E} 1$ & 17 & . & . & 55 & 11 & . & . & 31 \\
\hline Dianthus sylvestris & $\mathrm{E} 1$ & 10 & 9 & . & . & . & . & . & . \\
\hline Kernera saxatilis & E1 & 4 & . & . & 2 & . & . & 11 & 31 \\
\hline Moehringia muscosa & E1 & 4 & . & . & . & . & . & . & . \\
\hline Heliosperma pusillum & $\mathrm{E} 1$ & 4 & . & . & . & . & . & . & . \\
\hline Asplenium viride & E1 & 2 & . & . & 5 & 11 & . & . & . \\
\hline Asplenium trichomanes & E1 & 2 & . & . & . & 5 & . & . & . \\
\hline Sedum album & E1 & . & . & . & 5 & 5 & . & . & . \\
\hline Carex brachystachys & E1 & . & . & . & 2 & . & . & . & . \\
\hline Erysimum sylvestre & E1 & . & . & . & 2 & . & . & . & . \\
\hline Polypodium vulgare & E1 & . & . & . & 2 & . & . & . & . \\
\hline \multicolumn{10}{|l|}{ Other species (Druge vrste) } \\
\hline Campanula sp. & E1 & 12 & . & . & . & . & . & . & . \\
\hline Euphorbia sp. & E1 & 4 & . & . & . & . & . & . & . \\
\hline Galium sp. & E1 & 4 & . & . & . & . & . & . & . \\
\hline Hieracium sp. & E1 & 2 & & 10 & 2 & . & . & . & . \\
\hline Carex sp. & E1 & 2 & . & . & . & . & . & . & . \\
\hline Viola sp. & E1 & 2 & . & . & . & . & . & . & . \\
\hline Festuca sp. & E1 & . & . & . & 2 & . & . & . & . \\
\hline Vicia angustifolia & $\mathrm{E} 1$ & . & . & . & . & 5 & . & . & . \\
\hline \multicolumn{10}{|l|}{ Mosses and lichens (Mahovi in lišaji) } \\
\hline Tortella tortuosa & E0 & 71,154 & . & 20 & 60 & . & . & 61 & 83 \\
\hline Scleropodium purum & E0 & 50 & 9 & 5 & 78 & . & 26 & 17 & . \\
\hline Rhytidiadelphus triquetrus & E0 & 40 & . & 30 & 16 & 16 & 15 & 39 & . \\
\hline Ctenidium molluscum & E0 & 25 & 9 & 5 & 38 & . & 4 & 17 & 14 \\
\hline Hylocomium splendens & E0 & 17 & . & 25 & 15 & 26 & 22 & 61 & . \\
\hline Dicranum scoparium & E0 & 17 & . & 10 & 4 & . & 19 & 28 & . \\
\hline Neckera crispa & E0 & 17 & . & . & 33 & 5 & . & . & . \\
\hline Schistidium sp. & E0 & 15 & . & . & 11 & . & . & . & 78 \\
\hline Pleurozium schreberi & E0 & 13 & . & . & 4 & 26 & 52 & 61 & . \\
\hline
\end{tabular}




\begin{tabular}{|c|c|c|c|c|c|c|c|c|c|}
\hline Successive number (Zaporedna številka) & & 1 & 2 & 3 & 4 & 5 & 6 & 7 & 8 \\
\hline Hypnum cupressiforme & E0 & 13 & . & . & 18 & 5 & . & . & . \\
\hline Fissidens dubius & E0 & 12 & . & . & 11 & . & . & 6 & 28 \\
\hline Isothecium alopecuroides & E0 & 6 & . & . & 2 & . & . & . & . \\
\hline Plagiochila porelloides & E0 & 6 & . & . & 2 & . & . & . & . \\
\hline Cladonia sp. & E0 & 4 & . & 15 & . & . & . & . & . \\
\hline Rhytidium rugosum & E0 & 4 & . & . & 4 & . & . & 39 & 8 \\
\hline Musci spp. & E0 & 2 & . & . & . & . & . & . & . \\
\hline Conocephalum conicum & E0 & 2 & . & . & . & . & . & . & . \\
\hline Leucobryum glaucum & E0 & 2 & . & . & 2 & . & . & . & . \\
\hline Cladonia rangiferina & E0 & 2 & . & . & . & . & . & . & $\cdot$ \\
\hline Dicranella heteromalla & E0 & 2 & . & . & . & . & . & . & . \\
\hline Hookeria lucens & E0 & 2 & . & . & . & . & . & . & . \\
\hline Marchantia polymorpha & E0 & 2 & . & . & . & . & . & . & . \\
\hline Solorina saccata & E0 & 2 & . & . & . & . & . & . & $\cdot$ \\
\hline Polytrichum formosum & E0 & 2 & . & . & . & . & . & . & $\cdot$ \\
\hline Cladonia pyxidata & E0 & 2 & . & . & . & . & . & . & . \\
\hline Pedinophyllum interruptum & E0 & . & 9 & . & . & . & . & . & . \\
\hline Cetraria islandica & E0 & . & . & 10 & . & . & . & . & $\cdot$ \\
\hline Thamnolia vermicularis & E0 & . & . & 5 & . & . & . & . & $\cdot$ \\
\hline Cladonia pyxidata & E0 & . & . & 5 & . & . & . & . & . \\
\hline Sphagnum rubellum & E0 & . & . & 5 & $\cdot$ & . & . & . & . \\
\hline Homalothecium lutescens & E0 & . & . & . & 11 & . & 7 & . & 22 \\
\hline Thuidium tamariscinum & E0 & . & . & . & 5 & . & . & . & . \\
\hline Homalothecium sericeum & E0 & . & . & . & 4 & . & . & . & . \\
\hline Thuidium abietinum & E0 & . & . & . & 4 & . & . & . & . \\
\hline Campylium stellatum & E0 & . & . & . & 2 & . & . & . & . \\
\hline Encalypta streptocarpa & E0 & . & . & . & 2 & . & . & . & $\cdot$ \\
\hline Eurhynchium striatum & E0 & . & . & . & 2 & . & . & . & $\cdot$ \\
\hline Hypnum jutlandicum & E0 & . & . & . & 2 & . & . & . & $\cdot$ \\
\hline Leoskebryum brevirostre & E0 & . & . & . & 2 & . & . & . & $\cdot$ \\
\hline Neckera complanata & E0 & . & . & . & 2 & . & . & . & $\cdot$ \\
\hline Thuidium delicatulum & E0 & . & . & . & 2 & . & . & . & . \\
\hline Dicranum polysetum & E0 & . & . & . & . &. & . & 50 &. \\
\hline
\end{tabular}

\section{Legend - Legenda}

RcPs-Si Rhodothamno-Pinetum sylvestris, this article, Table 1

FPnps Fraxino orni-Pinetum nigrae pinetosum sylvestris, NW Italy, Poldini \& Vidali 1999, Table 2, relevés 18-28;

EPs-Do Erico-Pinetum sylvestris, Dolomites, E. \& S. Pignatti 2016, Association Table 5.2;

EPsoc-K Erico-Pinetum sylvestris ostryetosum carpinifoliae, Carinthia, Austra, Franz 2002, Table 34;

EPspm Erico-Pinetum sylvestris pinetosum mugo, Austria, Eichberger et al. 2007 b, Table 29, Column 4;

EPsch Erico-Pinetum sylvestris caricetosum humilis, Austria, Eichberger et al. 2007 b, Table 29, Column 2;

EPsoc-A Erico-Pinetum sylvestris ostryetosum carpinifoliae, Austria, Eichberger et al. 2007 b, Table 29, Column 7. 
Table 4: Phytosociological groups in the syntaxa Rhodothamno-Pinetum sylvestris, Fraxino orni-Pinetum nigrae pinetosum sylvestris, Erico-Pinetum sylvestris and Genisto-Pinetum sylvestris (relative frequencies).

Tabela 4: Skupine diagnostičnih vrst v sintaksonih Rhodothamno-Pinetum sylvestris, Fraxino orni-Pinetum nigrae pinestoum sylvestris, Erico-Pinetum sylvestris in Genisto-Pinetum sylvestris (relativne frekvence).

\begin{tabular}{|c|c|c|c|c|c|c|c|c|}
\hline Successive number (Zaporedna številka) & 1 & 2 & 3 & 4 & 5 & 6 & 7 & 8 \\
\hline Sign for syntaxa (Oznaka sintaksonov) & $\dot{\tilde{s}}_{\tilde{c}}$ & 总 & $\stackrel{\dot{m}}{\dot{\mid c}}$ & 心ٌ & 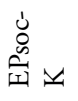 & 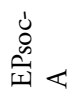 & $\begin{array}{c}E \\
\frac{\Xi}{n} \\
\hat{n}_{1}\end{array}$ & 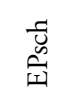 \\
\hline Authors (Avtorji) & $\stackrel{Q}{\frac{\alpha}{\alpha}} \sum_{j}^{N}$ & $\sum_{\Xi}$ & 吕 & 으 & $\stackrel{u}{\lessgtr}$ & 甹 & 吾 & 吾 \\
\hline Number of releves & 53 & 11 & 20 & 55 & 19 & 27 & 18 & 36 \\
\hline Erico-Pinetea & 23.18 & 28.9 & 24.5 & 20.3 & 19.5 & 24.4 & 26 & 26.5 \\
\hline Vaccinio-Piceetea & 11.74 & 9.85 & 8.55 & 3.6 & 13 & 13.1 & 14.2 & 5.87 \\
\hline Aremonio-Fagion & 4.1 & 3.66 & 0.74 & 3.82 & 5.84 & 5.39 & 0.16 & 0.09 \\
\hline Tilio-Acerion & 0.93 & 0.4 & 0.12 & 2.38 & 2.7 & 0.73 & 0.16 & 0.58 \\
\hline Alnion incanae, Salicetea purpureae & 0.08 & 0.6 & 0.5 & 0.96 & 0.34 & 0.85 & 0.28 & 0 \\
\hline Fagetalia sylvaticae & 5.61 & 5.42 & 3.47 & 3.58 & 14.1 & 5.59 & 1.6 & 2.06 \\
\hline Quercetalia pubescenti-petraeae & 4.06 & 4.23 & 2.23 & 11.9 & 14 & 7.17 & 2.84 & 2.83 \\
\hline Quercetalia roboris-petraeae & 1.67 & 4.64 & 1.49 & 2.3 & 3.71 & 3.89 & 3.02 & 0.58 \\
\hline Querco-Fagetea & 1.89 & 3.41 & 3.1 & 3 & 3.78 & 2.89 & 0.59 & 0.25 \\
\hline Sambuco-Salicion capreae & 0.97 & 1.6 & 0.5 & 0.12 & 1.45 & 0.58 & 0.28 & 1.2 \\
\hline Rhamno-Prunetea & 1.23 & 2.83 & 2.97 & 5.1 & 2.5 & 7.44 & 2.87 & 0.77 \\
\hline Trifolio-Geranietea & 2.83 & 3.41 & 3.35 & 4.96 & 1.75 & 2.43 & 1.01 & 5.84 \\
\hline Betulo-Alnetea, Mulgedio-Aconitetea & 1.39 & 1.4 & 2.97 & 0.78 & 0.34 & 0 & 2.3 & 0.25 \\
\hline Epilobietea angustifolii & 0.29 & 0.4 & 0.62 & 0.12 & 0.17 & 0.58 & 0.16 & 0.77 \\
\hline Elyno-Seslerietea & 11 & 5.26 & 14.7 & 4.38 & 1.45 & 1.43 & 14.6 & 15.1 \\
\hline Caricetalia davallianae & 0.33 & 0.4 & 1.12 & 0.46 & 0.17 & 0 & 0 & 0 \\
\hline Calluno-Ulicetea, Juncetea trifidi & 0.22 & 0.6 & 0.37 & 0 & 0.54 & 0.15 & 0.16 & 0.18 \\
\hline Festuco-Brometea & 11.1 & 16.3 & 14.1 & 15.8 & 5.47 & 13.8 & 12.8 & 15.7 \\
\hline Molinio-Arrhenatheretea & 2.25 & 2.21 & 2.48 & 0.78 & 0.71 & 2.62 & 2.59 & 2.98 \\
\hline Thlaspietea rotundifolii & 3.92 & 2.01 & 6.82 & 4.26 & 3.88 & 1.43 & 2.59 & 1.72 \\
\hline Potentilletalia caulescentis, Physoplexido-Saxifragion & 3.77 & 1.63 & 1.61 & 3.1 & 0.71 & 0 & 1.6 & 7.68 \\
\hline Asplenietea trichomanis & 0.8 & 0.2 & 0 & 1.46 & 1.08 & 0 & 0.28 & 1.91 \\
\hline Other species (Druge vrste) & 0.47 & 0 & 0.25 & 0.08 & 0.17 & 0 & 0 & 0 \\
\hline Mosses and lichens (Mahovi in lišaji) & 6.23 & 0.6 & 3.35 & 6.72 & 2.63 & 5.59 & 9.8 & 7.16 \\
\hline Total (Skupaj) & 100 & 100 & 100 & 100 & 100 & 100 & 100 & 100 \\
\hline
\end{tabular}

Legend - Legenda - see Table 3 - glej Preglednico 3

Table 5: The number of indicator species by phytosociological groups in both subassociations of the association RhodothamnoPinetum sylvestris.

Tabela 5: Število indikatorskih vrst v fitosocioloških skupinah v obeh subasociacijah asociacije Rhodothamno-Pinetum sylvestris.

\begin{tabular}{|c|c|c|c|c|c|}
\hline IndVal analysis, $\mathrm{p}$-val $<0.05$ & euphorbietosum & sorbetosum & IndVal analysis, $\mathrm{p}$-val $<\mathbf{0 . 0 5}$ & euphorbietosum & sorbetosum \\
\hline Aremonio-Fagion & 1 & & Quercetalia pubescenti-petraeae & 1 & \\
\hline Asplenietea trichomanis & & 1 & Quercetalia roboris-petraeae & 1 & 1 \\
\hline Elyno-Seslerietea & & 6 & Querco-Fagetea & 1 & \\
\hline Erico-Pinetea & 7 & 7 & Rhamno-Prunetea & & 1 \\
\hline Fagetalia sylvaticae & 2 & 1 & Sambuco-Salicion capreae & & 2 \\
\hline Festuco-Brometea & 8 & 1 & Thlaspietea rotundifolii & & 2 \\
\hline Molinio-Arrhenatheretea, Molinion & 1 & & Tilio-Acerion & & 1 \\
\hline $\begin{array}{l}\text { Potentilletalia caulescentis, } \\
\text { Physoplexido-Saxifragion }\end{array}$ & 2 & 1 & $\begin{array}{l}\text { Trifolio-Geranietea } \\
\text { Vaccinio-Piceetea }\end{array}$ & 3 & \\
\hline & & & Vaccinio-Piceetea & & 4 \\
\hline
\end{tabular}

